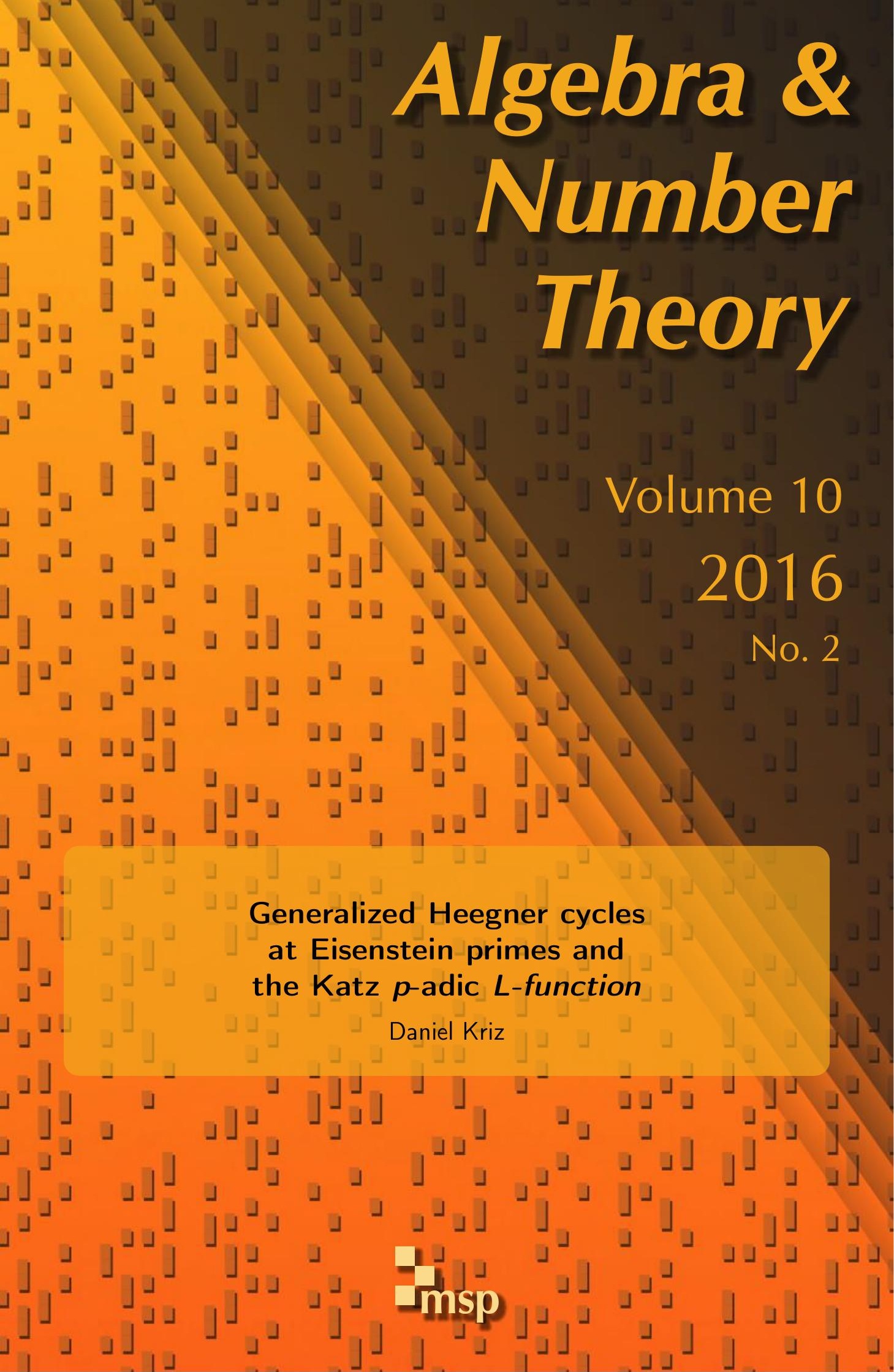




\title{
Generalized Heegner cycles at Eisenstein primes and the Katz $p$-adic L-function
}

\author{
Daniel Kriz
}

We consider normalized newforms $f \in S_{k}\left(\Gamma_{0}(N), \varepsilon_{f}\right)$ whose nonconstant term Fourier coefficients are congruent to those of an Eisenstein series modulo some prime ideal above a rational prime $p$. In this situation, we establish a congruence between the anticyclotomic $p$-adic $L$-function of Bertolini, Darmon, and Prasanna and the Katz two-variable $p$-adic $L$-function. From this we derive congruences between images under the $p$-adic Abel-Jacobi map of certain generalized Heegner cycles attached to $f$ and special values of the Katz $p$-adic $L$-function.

Our results apply to newforms associated with elliptic curves $E / \mathbb{Q}$ whose mod- $p$ Galois representations $E[p]$ are reducible at a good prime $p$. As a consequence, we show the following: if $K$ is an imaginary quadratic field satisfying the Heegner hypothesis with respect to $E$ and in which $p$ splits, and if the bad primes of $E$ satisfy certain congruence conditions $\bmod p$ and $p$ does not divide certain Bernoulli numbers, then the Heegner point $P_{E}(K)$ is nontorsion, implying, in particular, that $\operatorname{rank}_{\mathbb{Z}} E(K)=1$. From this we show that if $E$ is semistable with reducible mod-3 Galois representation, then a positive proportion of real quadratic twists of $E$ have rank 1 and a positive proportion of imaginary quadratic twists of $E$ have rank 0 .

1. Notation and conventions 310

2. Introduction 312

3. Preliminaries 327

4. Proof of the main theorem 344

5. Concrete applications of the main theorem 354

Acknowledgements 371

$\begin{array}{ll}\text { References } & 372\end{array}$

MSC2010: primary 11G40; secondary 11G05, 11G15, 11G35.

Keywords: Heegner cycles, $p$-adic Abel-Jacobi map, Katz $p$-adic $L$-function, Beilinson-Bloch conjecture, Goldfeld's conjecture. 


\section{Notation and conventions}

Throughout the paper, let us fix the following notational conventions.

For $m, n \in \mathbb{Z}$, let $(m, n)$ denote the greatest common divisor of $m$ and $n$, and let $\operatorname{lcm}(m, n)$ denote the least common multiple. We let $\ell \| N$ indicate that $\ell$ strictly divides $N$. We let $\Phi: \mathbb{Z} \rightarrow \mathbb{Z}$ denote the Euler totient function. Given an extension of number fields $L / K$ and an integral ideal $\mathfrak{a}$ of $\mathcal{O}_{L}$, let $\mathrm{Nm}_{L / K} \mathfrak{a}$ denote the relative ideal norm of $\mathfrak{a}$ and let $|\mathfrak{a}|$ denote the smallest positive rational integer in $\mathfrak{a}$. For ideals $\mathfrak{a}, \mathfrak{b}$, we let $(\mathfrak{a}, \mathfrak{b})$ denote the greatest common ideal divisor and $\operatorname{lcm}(\mathfrak{a}, \mathfrak{b})$ the least common ideal multiple. For a place $v$ of a number field, let Frob ${ }_{v}$ denote the arithmetic Frobenius attached to $v$, i.e., the Frobenius element which is sent to $v$ under the (inverse of the) Artin reciprocity map.

Throughout, we will fix an algebraic closure $\overline{\mathbb{Q}}$ of $\mathbb{Q}$. All number fields in our discussion will be viewed as being embedded in $\overline{\mathbb{Q}}$. For each rational prime $p$, we will fix an algebraic closure $\overline{\mathbb{Q}}_{p}$ of $\mathbb{Q}_{p}$ and let $\mathbb{C}_{p}$ denote the topological closure of $\overline{\mathbb{Q}}_{p}$. We fix an embedding $i_{\infty}: \overline{\mathbb{Q}} \hookrightarrow \mathbb{C}$ as well as an embedding $i_{p}: \overline{\mathbb{Q}} \hookrightarrow \mathbb{C}_{p}$ for each $p$. We also fix field identifications $i: \mathbb{C} \stackrel{\sim}{\longrightarrow} \mathbb{C}_{p}$ for each $p$. (We will use the same symbol $i$ for all $p$, as the underlying $p$ will be clear from context.)

Let $K$ denote a general number field. Let $\mathbb{A}_{K}$ and $\mathbb{A}_{K}^{\times}$denote the adèles and idèles over $K$, respectively, and let $\mathbb{A}_{K, f}$ and $\mathbb{A}_{K, f}^{\times}$denote the finite adèles and idèles, respectively. For a Hecke character $\chi: \mathbb{A}_{K}^{\times} \rightarrow \mathbb{C}^{\times}$, let $\mathfrak{f}(\chi) \subset \mathcal{O}_{K}$ denote the conductor of $\chi$. As usual, if $\mathfrak{a} \subset \mathcal{O}_{K}$ is an integral ideal with $(\mathfrak{a}, \mathfrak{f}(\chi)) \neq 1$, then we set $\chi(\mathfrak{a})=0$.

Given a Dirichlet (i.e., finite order) character $\psi$ over a number field $K$ of conductor $\mathfrak{f} \subset \mathcal{O}_{K}$, we will identify $\psi$ with its associated finite order Hecke character on idèles, taking the following convention: for $x \in(\mathcal{O} / \mathfrak{f})^{\times}$,

$$
\psi(x \bmod \mathfrak{f})=\prod_{v \nmid \mathfrak{f}} \psi_{v}(x)=\prod_{v \mid \mathfrak{f}} \psi_{v}^{-1}(x)
$$

where, in the first product, $v$ runs over all places of $E$ which do not divide $\mathfrak{f}$. When $K=\mathbb{Q}$, we define the Gauss sum of $\psi$ by

$$
\mathfrak{g}(\psi):=\sum_{a \in(\mathbb{Z} / \mathfrak{f} \mathbb{Z})^{\times}} \psi(a) e^{2 \pi i(a / \mid \mathfrak{f l})}
$$

For a finite prime $\ell$, we define the Gauss sum of the local character $\psi_{\ell}: \mathbb{Q}_{\ell}^{\times} \rightarrow \mathbb{C}^{\times}$ similarly: letting $|\mathfrak{f}|=\prod_{\ell} \ell^{e_{\ell}}$, we set

$$
\mathfrak{g}_{\ell}(\psi):=\psi_{\ell}\left(\ell^{e_{\ell}}\right) \sum_{a \in\left(\mathbb{Z}_{\ell} / \ell^{\ell_{\ell}} \mathbb{Z}_{\ell}\right)^{\times}} \psi_{\ell}^{-1}(a) e^{2 \pi i\left\{a / \ell^{e}\right\}_{\ell}},
$$


where $\{x\}_{\ell}$ denotes the $\ell$-fractional part of $x \in \mathbb{Z}_{\ell}$. Thus

$$
\prod_{\ell \mid \mathfrak{f}} \mathfrak{g}_{\ell}(\psi)=\mathfrak{g}(\psi) \prod_{\ell \mid \mathfrak{f}} \psi_{\ell}(|\mathfrak{f}|)=\mathfrak{g}(\psi) \prod_{\ell \nmid \mathfrak{f}} \psi_{\ell}^{-1}(|\mathfrak{f}|)=\mathfrak{g}(\psi) .
$$

Let $\mathbb{N}_{\mathbb{Q}}: \mathbb{A}_{\mathbb{Q}} \rightarrow \mathbb{C}$ denote the adèlic norm, normalized so that $\mathbb{N}_{\mathbb{Q}, \infty}$ equals $|\cdot|_{\infty}$ (the usual archimedean absolute value). Then, for any number field $K$, let $\mathrm{Nm}_{K / \mathbb{Q}}: \mathbb{A}_{K} \rightarrow \mathbb{A}_{\mathbb{Q}}$ denote the norm homomorphism (which induces the ideal norm recalled above), and set $\mathbb{N}_{K}:=\mathbb{N}_{\mathbb{Q}} \circ \mathrm{Nm}_{K / \mathbb{Q}}$. Note that when $K$ is imaginary quadratic, $\mathbb{N}_{K}: \mathbb{A}_{K}^{\times} \rightarrow \mathbb{C}^{\times}$is an algebraic Hecke character of infinity type $(-1,-1)$. For any Hecke character $\chi: \mathbb{A}_{K}^{\times} \rightarrow \mathbb{C}^{\times}$, let

$$
\chi_{j}:=\chi \mathbb{N}_{K}^{-j} .
$$

Using the fixed isomorphism $i: \mathbb{C} \stackrel{\sim}{\longrightarrow} \mathbb{C}_{p}$ and the Artin isomorphism, we can view $\mathbb{N}_{K}^{-1}$ as a character $\mathbb{N}_{K}^{-1}: \operatorname{Gal}(\bar{K} / K) \rightarrow \mathbb{Z}_{p}^{\times}$. Then any $x \in \mathbb{Z}_{p}^{\times}$can be uniquely written as $\omega(x) \cdot\langle x\rangle$, where $\omega(x) \in \mu_{2(p-1)}$ and $\langle x\rangle \in 1+2 p \mathbb{Z}_{p}$. We then define the Teichmüller character $\omega: \operatorname{Gal}(\bar{K} / K) \rightarrow \mu_{2(p-1)}$ by $\omega_{K}(a):=\omega\left(\mathbb{N}_{K}^{-1}(a)\right)$. For simplicity, we will let $\omega_{\mathbb{Q}}=\omega$. Given an extension of number fields $K / L$ and a Hecke character $\phi$ over $L$, we will let $\phi_{/ K}:=\phi \circ \mathrm{Nm}_{K / L}$; note that, viewing $\phi$ as a character $\operatorname{Gal}(\bar{L} / L) \rightarrow \mathbb{C}^{\times}$, this corresponds to restriction to the subgroup $\operatorname{Gal}(\bar{K} / K)$.

For a quadratic field $K / \mathbb{Q}$ with $K=\mathbb{Q}(\sqrt{D})$, where $D$ is a squarefree integer, we recall that the fundamental discriminant of $K$ is given by

$$
D_{K}= \begin{cases}D & \text { if } D \equiv 1(\bmod 4) \\ 4 D & \text { if } D \equiv 2,3(\bmod 4) .\end{cases}
$$

For quadratic $K$, let $\varepsilon_{K}$ be the associated Dirichlet character of conductor $D_{K}$; conversely, for a quadratic Dirichlet character $\chi$, let $K_{\chi}$ be the associated imaginary quadratic field. Given two quadratic fields $L=\mathbb{Q}(\sqrt{D})$ and $K=\mathbb{Q}\left(\sqrt{D^{\prime}}\right)$, we will let $L \cdot K$ denote the quadratic field $\mathbb{Q}\left(\sqrt{D D^{\prime}}\right)$. Unless otherwise noted, all Dirichlet characters over $\mathbb{Q}$ are taken to be primitive, and so are uniquely identified with finite order Hecke characters over $\mathbb{Q}$ under the arithmetic normalization described above. Given Dirichlet characters $\psi_{1}, \psi_{2}$ over $\mathbb{Q}$, we define $\psi_{1} \psi_{2}$ to be the primitive Dirichlet character equal to $\psi_{1}(a) \psi_{2}(a)$ for $a \in\left(\mathbb{Z} / \operatorname{lcm}\left(\mathfrak{f}\left(\psi_{1}\right), \mathfrak{f}\left(\psi_{2}\right)\right)\right)^{\times}$ (and indeed this equality holds for the associated Hecke characters). In particular, for quadratic $L$ and $K$ as above, we have $\varepsilon_{L \cdot K}=\varepsilon_{L} \varepsilon_{K}$.

Given a normalized newform $f \in S_{k}\left(\Gamma_{1}(N)\right)$, let $a_{n}(f)$ denote the $n$-th Fourier coefficient of $f$ (i.e., the $n$-th coefficient of the $q$-expansion at $\infty$ ); when $f$ is obvious from context, we will often abbreviate $a_{n}(f)$ to $a_{n}$. Moreover, when $f$ is defined over $\mathbb{Q}$ and thus associated with an elliptic curve $E / \mathbb{Q}$, we will sometimes write $a_{n}(E)=a_{n}(f)$. Let $E_{f}$ denote the finite extension of $\mathbb{Q}$ generated by the 
Fourier coefficients $a_{n}(f)$. Given a Hecke character $\chi$, let $E_{\chi}$ denote the finite extension of $\mathbb{Q}$ generated by its values. Set $E_{f, \chi}=E_{f} E_{\chi}$.

For $s \in \mathbb{C}$, let $\Re(s)$ and $\Im(s)$ denote the real and imaginary parts of $s$, respectively. We let $\mathcal{H}^{+}:=\{s \in \mathbb{C}: \Im(s)>0\}$.

\section{Introduction}

The study of Heegner points has provided some of the greatest insights into the Birch and Swinnerton-Dyer conjecture. In particular, given an elliptic curve $E / \mathbb{Q}$ and an imaginary quadratic field $K$ satisfying a suitable Heegner hypothesis with respect to $E$, the nontriviality of the Heegner point $P_{E}(K) \in E(K) \otimes \mathbb{Q}$ introduced in Section 3.4 implies, via the descent argument of [Kolyvagin 1990], that $\operatorname{rank}_{\mathbb{Z}} E(K)=1$. Our main result establishes, for elliptic curves $E$ with $E[p]$ a reducible Galois representation at a good prime $p$, a congruence mod $p$ between the formal logarithm of the Heegner point and a special value of the Katz $p$-adic $L$-function with certain Euler factors removed. Using Gross's factorization [1980] of the Katz $p$-adic $L$-function on the cyclotomic line, we can then find an explicit congruence between the formal logarithm of the Heegner point and a quantity involving certain Bernoulli numbers and (inverses of) Euler factors at primes of bad reduction. Thus we derive an explicit criterion (involving Bernoulli numbers) for the nontriviality of $P_{E}(K) \in E(K) \otimes \mathbb{Q}$ in certain families of $E$.

Our results also have higher-dimensional applications, namely in computing with algebraic cycle classes in Chow groups of motives attached to newforms. Suppose $N$ is a positive integer and $K$ is an imaginary quadratic field satisfying the Heegner hypothesis with respect to $N$, suppose $f=\sum_{n \geq 1} a_{n} q^{n}$ is a weight- $k \geq 2$ level- $N$ normalized newform whose Fourier coefficients are congruent to those of an Eisenstein series outside the constant term modulo some prime ideal above $p$, and suppose $\chi$ is an algebraic Hecke character over $K$ central critical with respect to $f$. We consider the Rankin-Selberg motive $M_{f, \chi^{-1}}:=M_{f_{/ K}} \otimes M_{\chi^{-1}}$, defined over $K$, associated with $(f, \chi)$, where $M_{f}$ is the motive associated with $f$ (see [Scholl 1990]), $M_{f_{/ K}}$ is its base change to $K$, and $M_{\chi}$ is the motive associated with $\chi$ (see [Deninger 1990]). For convenience of notation, we will formulate our discussion with respect to

$$
M_{\left(f, \chi^{-1}\right)_{/ F}}=M_{f_{/ F}} \otimes M_{\left(\chi^{-1}\right)_{/ F}},
$$

where $F / K$ is some large enough abelian extension in "situation $(S)$ " as described on page 314 , and $f_{/ F}$ and $\chi_{/ F}$ are the corresponding base extensions to $F$. In fact, one may recover $M_{\chi^{-1}}$ as a direct factor of the Grothendieck restriction of $M_{\left(\chi^{-1}\right)_{/ F}}$; see [Deninger 1990] or [Geisser 1997]. Indeed, we have a factorization

$$
M_{\left(f, \chi^{-1}\right)_{/ F}}=\bigoplus_{\chi_{0}: \operatorname{Gal}(F / K) \rightarrow \mathbb{C}^{\times}} M_{f, \chi^{-1} \chi_{0}} .
$$


Under certain conditions guaranteeing that the value of $L\left(\pi_{f} \times \pi_{\chi^{-1}}, s\right)=$ $L\left(M_{f, \chi^{-1}}, s\right)$ (and thus also that of $L\left(\left(\pi_{f} \times \pi_{\chi^{-1}}\right)_{/ F}, s\right)=L\left(M_{\left(f, \chi^{-1}\right)_{/ F}}, s\right)$ ) will vanish at the central point of the functional equation, we seek to construct algebraic cycle classes in the Chow group associated with $M_{\left(f, \chi^{-1}\right)_{/ F}}$, thus providing evidence for the Beilinson-Bloch conjecture for Chow motives associated with $(f, \chi)_{/ F}$. Natural candidates for representatives of such classes are generalized Heegner cycles (in the sense of [Bertolini et al. 2013]), generated by algebraic cycles on a suitable generalized Kuga-Sato variety which arise from graphs of isogenies between products of $\mathrm{CM}$ curves. Under the above hypotheses, our main theorem provides a criterion for a generalized Heegner cycle class associated with $(f, \chi)$, with $\chi$ in a certain infinity type range, to be nontrivial, namely that an associated special value of the Katz $p$-adic $L$-function (with certain Euler factors removed) is not (too) divisible by $p$. One may thus use special value formulas of $p$-adic $L$-functions in order to get explicit $p$-nondivisibility criteria for the nontriviality of Heegner cycle classes. In our main corollary, we explicitly address the case when Gross's factorization theorem can be applied, which corresponds precisely with the case when the relevant generalized Heegner cycle arises from a classical Heegner cycle (see Remark 8).

2.1. Chow motives. To make our discussion more precise, let us briefly recall some definitions pertaining to Chow motives. Let $X$ be a nonsingular variety over a number field $F$. An algebraic cycle of $X$ is a formal sum of subvarieties of $X$. We let $\mathrm{CH}^{j}(X)$ denote the group generated by algebraic cycles of $X$ of codimension $j$ modulo rational equivalence. Call $\mathrm{CH}^{j}(X)$ the $j$-th Chow group of $X$, and call $\mathrm{CH}(X):=\bigoplus_{0 \leq j \leq \operatorname{dim} X} \mathrm{CH}^{j}(X)$ the Chow group of $X$. For varieties $X, Y$ over $F$, call $\operatorname{Corr}^{d}(X, Y):=\mathrm{CH}^{\operatorname{dim} Y+d}\left(X \times_{F} Y\right)$ the group of (degree-d) correspondences from $X$ to $Y$. The group $\operatorname{Corr}(X, X):=\bigoplus_{0 \leq d \leq \operatorname{dim} X} \operatorname{Corr}^{d}(X, X)$ has a ring structure defined by composition of correspondences: given $g \in \operatorname{Corr}^{d_{1}}(X, Y)$ and $h \in \operatorname{Corr}^{d_{2}}(Y, Z)$, we define

$$
h \circ g:=\pi_{13, *}\left(\pi_{12}^{*}(g) . \pi_{23}^{*}(h)\right) \in \operatorname{Corr}^{d_{1}+d_{2}}(X, Z),
$$

where $\pi_{12}, \pi_{13}, \pi_{23}: X \times_{F} Y \times_{F} Z \rightarrow X \times_{F} Y, X \times_{F} Z, Y \times_{F} Z$ are the canonical projections, and "." denotes the Chow intersection pairing. We call $\operatorname{Corr}(X, X)$ the ring of correspondences on $X$. For any number field $E$, let $\operatorname{Corr}^{d}(X, Y)_{E}:=$ $\operatorname{Corr}^{d}(X, Y) \otimes_{\mathbb{Z}} E$. A Chow motive over $F$ with coefficients in $E$ is a triple $(X, e, m)$ consisting of a nonsingular variety $X$ over $F$, an idempotent $e \in \operatorname{Corr}^{0}(X, X)_{E}$, and an integer $m$. Define the category of Chow motives $\mathcal{M}_{F, E}$ whose objects consist of Chow motives over $F$ with coefficients in $E$, and with morphisms given by

$$
\operatorname{Hom}_{\mathcal{M}_{F, E}}((X, e, m),(Y, f, n)):=f \circ \operatorname{Corr}^{n-m}(X, Y)_{\mathbb{Q}} \circ e .
$$


Define the category of Grothendieck motives $\mathcal{M}_{F, E}^{\text {hom }}$ with objects being cycles of $X$ modulo (motivic) homological equivalence, and with morphisms defined in the same way as above. Note that since rational equivalence is stronger than homological equivalence, there is a natural functor

$$
\mathcal{M}_{F, E} \rightarrow \mathcal{M}_{F, E}^{\text {hom }}
$$

Let $\mathrm{CH}^{j}(X)_{0}$ denote the subgroup of $\mathrm{CH}^{j}(X)$ consisting of null-homologous cycles.

2.2. The Beilinson-Bloch conjecture for Rankin-Selberg motives. Now let $K$ be an imaginary quadratic field, and let $H$ denote the Hilbert class field over $K$. Fix $f \in S_{k}\left(\Gamma_{0}(N), \varepsilon_{f}\right)$, where $k \geq 2$, and let $r:=k-2$. Let $\chi$ be a Hecke character over $K$ of infinity type $(r-j, j)$ which is central critical with respect to $f$, and where $0 \leq j \leq r$. Suppose also that every prime $\ell \mid N$ splits in $K$, i.e., $K$ satisfies the Heegner hypothesis with respect to $N$. Then we can choose an ideal $\mathfrak{N}$ of $\mathcal{O}_{K}$ with $\mathcal{O}_{K} / \mathfrak{N}=\mathbb{Z} / N$. Assume also that $\mathfrak{f}(\chi) \mid \mathfrak{N}$.

The ambient motive in our setup will be $M:=\left(X_{r}, \epsilon_{X}, 0\right) \in \mathcal{M}_{F, E_{f, \chi}}$, where $F$, to be fixed later, is some large enough abelian extension of $K$ containing $H$, and $\epsilon_{X}$ is some projector in the ring of correspondences on $X$ which has induced actions on the various cohomological realizations of $M$ via the corresponding cycle class maps. (The projector $\epsilon_{X}$ essentially picks out the part of the cohomology of $X_{r}$ which comes from the Galois representations attached to pairs $(f, \chi)_{/ F}$.) Here the underlying $((2 r+1)$-dimensional $)$ variety is the generalized Kuga-Sato variety $X_{r}:=W_{r} \times A^{r}$, defined over $H$, where $A$ is a fixed CM elliptic curve of $\mathcal{O}_{K}$-type (i.e., with $\operatorname{End}_{H}(A)=\mathcal{O}_{K}$ ) and $W_{r}:=\mathcal{E}^{r}$ (the classical Kuga-Sato variety) is the (canonical desingularization of the) $r$-fold fiber product of copies of the universal elliptic curve $\mathcal{E}$ with $\Gamma_{1}(N)$-level structure over the (compactified) modular curve $X_{1}(N):=\overline{Y_{1}(N)}$ (and thus is defined over $\mathbb{Q}$ ). This is well-defined in the case where the cusps of $Y_{1}(N)$ are regular in the sense of [Diamond and Shurman 2005, Section 3.2], which holds in particular when $N>4$. The fibers of $X_{r} \rightarrow X_{1}(N)$, outside of the cusps of $X_{1}(N)$, are of the form $E^{r} \times A^{r}$ where $E$ varies over elliptic curves.

The motive $M_{f}$ is given by the triple $\left(W_{r}, \epsilon_{f}, 0\right)$ for some projector in the ring of correspondences on $W_{r}$ which picks out the $f$-isotypic component of the cohomology of $W_{r}$ under the Hecke action; in particular, this Chow motive is defined over $\mathbb{Q}$, with coefficients in $E_{f}$. For an extension $F / \mathbb{Q}$, we let $M_{f_{/ F}}$ denote the base change to $F$. The definition of the motive $M_{\left(\chi_{j}^{-1}\right) / F}$ is slightly more subtle. Let $F / K$ be an abelian extension such that $\chi_{A}^{r-j} \bar{\chi}_{A}^{j}=\chi_{/ F}$, where $\chi_{A}: \mathbb{A}_{F}^{\times} \rightarrow K^{\times}$is the Hecke character associated with the CM elliptic curve $A$ having infinity type $(1, \ldots, 1,0, \ldots, 0)$ (with the first $[F: K]$ places corresponding to the embeddings $F \hookrightarrow \overline{\mathbb{Q}}$ preserving our fixed embedding $K \hookrightarrow \overline{\mathbb{Q}}$, and the next $[F: K]$ places to their complex conjugates). We then say $F$ is in "situation $(S)$ ". 
(Such an $F$ always exists; see [Deninger 1990, Proposition 1.3.1].) We have a motive $M_{\left(\chi^{-1}\right)_{F}}:=\left(A^{r}, \epsilon_{\chi / F}, 0\right) \in \mathcal{M}_{F, E_{\chi}}$ for some suitable projector $\epsilon_{\chi_{/ F}}$ picking out the $\chi_{/ F}=\chi_{A}^{r-j} \bar{\chi}_{A}^{j}$-isotypic component of the cohomology of $A^{r}$ under the Galois action.

Let $H_{\mathfrak{N}}$ be the field over which the individual points of $A[\mathfrak{N}]$ are defined. We will henceforth take and fix an abelian extension $F / K$ large enough so that it contains $H_{\mathfrak{N}}$ and is in situation $(S)$. (Note that this is possible since $H_{\mathfrak{N}}$ is an abelian extension of $K$.) The Rankin-Selberg motive

$$
M_{\left(f, \chi^{-1}\right)_{/ F}}:=M_{f_{/ F}} \otimes M_{\left(\chi^{-1}\right)_{/ F}}=\left(W_{r}, \epsilon_{f_{/ F}}, 0\right) \otimes\left(A^{r}, \epsilon_{\chi / F}, 0\right)=\left(X_{r}, \epsilon_{(f, \chi)_{/ F}}, 0\right)
$$

is a submotive of $M$ (in fact, it is the $(f, \chi)_{/ F}$-isotypic component $\epsilon_{(f, \chi)_{/ F}} M$ ), and the Beilinson-Bloch conjecture predicts that

$$
\begin{aligned}
\operatorname{dim}_{E_{f, \chi}} \epsilon_{(f, \chi)_{F}} \mathrm{CH}^{r+1}\left(X_{r}\right)_{0, E_{f, \chi}}(F) & =\operatorname{ord}_{s=r+1} L\left(H_{\mathrm{et}}^{2 r+1}\left(M_{\left(f, \chi^{-1}\right)_{/ F}}\right), s\right) \\
& =\operatorname{ord}_{s=r+1} L\left(\epsilon_{(f, \chi)_{/ F}} H_{\mathrm{ét}}^{2 r+1}(M), s\right) \\
& =\operatorname{ord}_{s=r+1} L\left(\left(V_{f_{/ K}} \otimes \chi^{-1}\right)_{/ F}(r+1), s\right) \\
& =\operatorname{ord}_{s=0} L\left(\left(V_{f_{/ K}} \otimes \chi^{-1}\right)_{/ F}, s\right) \\
& =\operatorname{ord}_{s=0} L\left(V_{f_{/ K}} \otimes \chi^{-1} \otimes \operatorname{Ind}_{F}^{K} 1, s\right) \\
& =\operatorname{ord}_{s=0} \prod_{\chi 0} L\left(V_{f_{/ K}} \otimes\left(\chi^{-1} \chi_{0}\right), s\right) \\
& \quad \operatorname{Gal}(F / K) \rightarrow \mathbb{C}^{\times} \\
= & \operatorname{ord}_{s=\frac{1}{2}} \prod_{\chi 0} L\left(\pi_{f} \times \pi_{\chi^{-1} \chi_{0}}, s\right) . \\
& \quad \operatorname{Gal}(F / K) \rightarrow \mathbb{C}^{\times}
\end{aligned}
$$

Here $\epsilon_{(f, \chi)_{/ F}}$ is the projector corresponding to the $(f, \chi)_{/ F}$-isotypic component of the cohomology of $X_{r}$ under the Hecke and Galois actions, $V_{f}$ is the 2-dimensional (unique semisimple) $\operatorname{Gal}\left(\overline{\mathbb{Q}} / \mathbb{Q}\right.$ )-representation associated with $f, V_{f_{/ K}}$ is its restriction to $\operatorname{Gal}(\bar{K} / K)$, and $\pi_{f}, \pi_{\chi^{-1}}$ are the automorphic representations (over $K$ ) associated with $f$ and $\theta_{\chi^{-1}}$ under the unitary normalizations.

Under the Heegner hypothesis, we have $L\left(\pi_{f} \times \pi_{\chi^{-1}}, \frac{1}{2}\right)=0$, and so the BeilinsonBloch conjecture predicts that

$$
\operatorname{dim}_{E_{f, \chi}} \epsilon_{(f, \chi)_{/ F}} \mathrm{CH}^{r+1}\left(X_{r}\right)_{0, E_{f, \chi}}(F) \geq 1 .
$$

Thus we should be able to find a null-homologous cycle class with nonvanishing $\epsilon_{(f, \chi)_{/ F}}$-isotypic component in the above Chow group. To this end, we can use the $p$-adic Waldspurger formula of Bertolini, Darmon, and Prasanna [Bertolini et al. 2013] to consider images under the $p$-adic Abel-Jacobi map of generalized Heegner cycles. Generalized Heegner cycles are, essentially, generated by graphs $\Gamma_{\varphi}$ of isogenies $\varphi: A \rightarrow A^{\prime}$ between CM elliptic curves with $\Gamma_{1}(N)$-level structure 
(i.e., such that $\operatorname{ker}(\varphi) \cap A[\mathfrak{N}]=0$ ). We can then view the graph $\Gamma_{\varphi}$ inside $X_{r}$ :

$$
\Gamma_{\varphi} \subset A^{r} \times\left(A^{\prime}\right)^{r} \subset A^{r} \times W_{r}=X_{r} .
$$

We define the associated generalized Heegner cycle associated with $(\varphi, A)$ as

$$
\Delta_{\varphi}:=\epsilon_{X} \Gamma_{\varphi} \in \mathrm{CH}^{r+1}\left(X_{r}\right)_{0, E_{f, \chi}}(F) .
$$

(See Section 3.3 for a precise definition.) It is also a fact that $\epsilon_{X} H^{2 r+2}\left(X_{r}, \mathbb{Q}\right)=0$, and so $\Delta_{\varphi}$ is indeed null-homologous. Generalized Heegner cycles are those cycles in $\mathrm{CH}^{r+1}\left(X_{r}\right)_{0, E_{f, \chi}}(F)$ generated by formal $E_{f, \chi}$-linear combinations of $\Delta_{\varphi}$ for varying $\varphi$. The space $\mathrm{CH}\left(X_{r}\right)_{0, E_{f, \chi}}(F)$ has an isotypic decomposition with respect to the action of the Hecke algebra (which is indexed by cuspidal eigenforms) and that of $\operatorname{Gal}(\bar{F} / F)$ (which is indexed by Hecke characters).

Let $F_{p}$ denote the $p$-adic completion of $F$ determined by our fixed embedding $i_{p}: \overline{\mathbb{Q}} \hookrightarrow \mathbb{C}_{p}$. We can now apply the $p$-adic Abel-Jacobi map over $F_{p}$ to $\Delta_{\varphi}$, which is a map

$$
\begin{aligned}
\mathrm{AJ}_{F_{p}}: \mathrm{CH}^{r+1}\left(X_{r}\right)_{0, \mathbb{Q}}\left(F_{p}\right) & \rightarrow\left(\mathrm{Fil}^{r+1} \epsilon_{X} H_{d R}^{2 r+1}\left(X_{r} / F_{p}\right)\right)^{\vee} \\
& \cong\left(S_{k}\left(\Gamma_{1}(N), F_{p}\right) \otimes \operatorname{Sym}^{r} H_{d R}^{1}\left(A / F_{p}\right)\right)^{\vee},
\end{aligned}
$$

where the superscript $\vee$ denotes the $F_{p}$-linear dual, and Fil ${ }^{j}$ denotes the $j$-th step of the Hodge filtration. A basis of $\epsilon_{X} H_{d R}^{2 r+1}\left(X_{r} / F\right)$ is given by elements of the form $\omega_{f} \wedge \omega_{A}^{j} \eta_{A}^{r-j}$ for $0 \leq j \leq r$, where

$$
\omega_{f} \in \mathrm{Fil}^{r+1} \epsilon_{X} H_{d R}^{r+1}\left(W_{r} / F\right) \cong S_{k}\left(\Gamma_{1}(N), F\right)
$$

is associated with $f \in S_{k}\left(\Gamma_{1}(N), F\right)$, and the $\omega_{A}^{j} \eta_{A}^{r-j}$ form a basis of

$$
\mathrm{Fil}^{r+1} \epsilon_{X} H_{d R}^{r}\left(A^{r} / F\right) \cong \operatorname{Sym}^{r} H_{d R}^{1}(A / F) .
$$

(Here $\omega_{A} \in \Omega^{1}(A / F)=H_{d R}^{1,0}(A / F)$ is a nowhere vanishing differential on $A$, and $\eta_{A} \in H_{d R}^{0,1}(A / F)$ is such that $\left\langle\omega_{A}, \eta_{A}\right\rangle=1$ under the cup product pairing on de Rham cohomology.)

Bertolini, Darmon, and Prasanna's $p$-adic Waldspurger formula relates a special value of an anticyclotomic $p$-adic $L$-function $\mathcal{L}_{p}(f, \chi)$ to the Abel-Jacobi image of a certain generalized Heegner cycle $\Delta$, evaluated at the basis element $\omega_{f} \wedge \omega_{A}^{j} \eta_{A}^{r-j}$. The dual basis element of this latter element is in the $(f, \chi)_{/ F}$-isotypic component of (Fil $\left.^{r+1} \epsilon_{X} H_{d R}^{2 r+1}\left(X_{r} / F\right)\right)^{\vee}$, and the idempotent $\epsilon_{(f, \chi)_{/ F}}$ induces the projection onto this $(f, \chi)_{/ F}$-isotypic component. By functoriality of projectors, the nonvanishing of $\operatorname{AJ}_{F_{p}}(\Delta)$ at $\omega_{f} \wedge \omega_{A}^{j} \eta_{A}^{r-j}$ shows the nontriviality of $\epsilon_{(f, \chi)_{/ F}} \Delta$. Hence, showing the nonvanishing of a special value of Bertolini, Darmon, and Prasanna's $p$-adic $L$-function verifies one consequence of the Beilinson-Bloch conjecture for the motive $M_{\left(f, \chi^{-1}\right)_{/ F}}=\left(X_{r}, \epsilon_{(f, \chi)_{/ F}}, 0\right)$. 
More precisely, nonvanishing of the anticyclotomic $p$-adic $L$ function $\mathcal{L}_{p}(f, \chi)$ and functoriality of the action of correspondences on $X_{r}$ imply

$$
\begin{aligned}
0 & \neq \mathrm{AJ}_{F_{p}}(\Delta)\left(\omega_{f} \wedge \omega_{A}^{j} \eta_{A}^{r-j}\right) \\
& =\mathrm{AJ}_{F_{p}}(\Delta)\left(\epsilon_{(f, \chi)_{/ F}}\left(\omega_{f} \wedge \omega_{A}^{j} \eta_{A}^{r-j}\right)\right) \\
& =\mathrm{AJ}_{F_{p}}\left(\epsilon_{(f, \chi)_{/ F}} \Delta\right)\left(\omega_{f} \wedge \omega_{A}^{j} \eta_{A}^{r-j}\right)
\end{aligned}
$$

and thus $0 \neq \epsilon_{(f, \chi)_{/ F}} \Delta \in \epsilon_{(f, \chi)_{\mid F}} \mathrm{CH}^{r+1}\left(X_{r}\right)_{0, E_{f, \chi}}(F)$.

The classical situation $r=0$ (i.e., $k=2$ ) is perhaps instructive. In this case, the underlying generalized Kuga-Sato variety is simply $X_{0}=X_{1}(N)$. Moreover, $\chi: \operatorname{Gal}(F / K) \rightarrow \mathbb{C}^{\times}$is of finite order, so that $\chi_{/ F}=1$. Therefore, we have $\mathrm{CH}^{1}\left(X_{1}(N)\right)_{0, E_{f, \chi}}(F)=J_{1}(N)_{E_{f, \chi}}(F)$. The $p$-adic Abel-Jacobi map

$$
\mathrm{AJ}_{F_{p}}: J_{1}(N)_{E_{f, \chi}}\left(F_{p}\right) \rightarrow S_{k}\left(\Gamma_{1}(N), F_{p}\right)_{E_{f, \chi}}^{\vee}
$$

is given by $P \mapsto\left(f \mapsto \log _{\omega_{f}} P\right)$, where $\log _{\omega_{f}}$ is the formal logarithm at $p$ associated with the nonvanishing differential $\omega_{f}$. Note that $\operatorname{Gal}(\overline{\mathbb{Q}} / \mathbb{Q})$ acts on modular forms (as one may see by letting $\mathrm{Gal}(\overline{\mathbb{Q}} / \mathbb{Q})$ act on the coefficients of $q$-expansions), and that this action also preserves eigenspaces of Hecke operators. For a modular form $f$, let $[f]$ denote its $\operatorname{Gal}(\overline{\mathbb{Q}} / \mathbb{Q})$-orbit, and let $S_{k}^{\text {new }}\left(\Gamma_{1}(N)\right)$ denote the space of $\Gamma_{1}(N)$-cuspidal newforms of weight $k$. Given a normalized newform $f$, EichlerShimura theory attaches to $f$ an abelian variety $A_{f}$ which arises as a quotient of $J_{1}(N)$. Let $A_{f, E}:=A_{f} \otimes_{E_{f}} E$ for any ring $E$ containing $E_{f}$. We have an isotypic decomposition of the component of $J_{1}(N)$ under the Hecke algebra action in the isogeny category,

$$
J_{1}(N)^{\text {new }} \sim \underset{\left\{[f], f \in S_{k}^{\text {new }}\left(\Gamma_{1}(N)\right)\right\}}{\sim} A_{f},
$$

which implies

$$
J_{1}(N)_{E_{f, \chi}}^{\text {new }}=\underset{\left\{[f], f \in S_{k}^{\text {new }}\left(\Gamma_{1}(N)\right)\right\}}{A_{f, E_{f, \chi}} .}
$$

We thus have natural surjections $\Phi_{f}: J_{1}(N) \rightarrow A_{f}$ (called modular parametrizations) and hence maps $\Phi_{f}: J_{1}(N)_{E_{f, \chi}}(F) \rightarrow A_{f, E_{f, \chi}}(F)$. Let $\chi_{0}: \operatorname{Gal}(F / K) \rightarrow \mathbb{C}^{\times}$. Denote by $\Phi_{\chi_{0}}: A_{f, E_{f, \chi}}(F) \rightarrow A_{f, E_{f, \chi}}(F)^{\chi_{0}}$ the projection onto the $\chi_{0}$-isotypic component under the action of $\operatorname{Gal}(F / K)$, and set $\Phi_{f, \chi_{0}}=\Phi_{\chi_{0}} \circ \Phi_{f}: J_{1}(N)_{E_{f, \chi}}(F) \rightarrow$ $A_{f, E_{f, \chi}}(F)^{\chi_{0}}$. Let $\omega_{A_{f}}$ be the unique invariant differential on the abelian variety $A_{f} / F$ satisfying $\Phi_{f}^{*} \omega_{A_{f}}=\omega_{f}$. Generalized Heegner cycles are simply classical Heegner points, i.e., degree-0 divisors $P\left(\chi_{0}\right) \in J_{1}(N)_{E_{f, \chi}}(F)$ arising from $\chi_{0}$-twisted $\mathrm{Gal}(F / K)$-traces of $\mathrm{CM}$ points on $X_{1}(N)(F)$. Therefore, $P_{f}\left(\chi_{0}\right):=$ $\Phi_{f, \chi_{0}}\left(P\left(\chi_{0}\right)\right) \in A_{f, E_{f, \chi}}(F)^{\chi_{0}}$. Note that, when $\chi_{0}=1$, we have $P_{f}(1) \in A_{f, E_{f, \chi}}(K)$. 
The nonvanishing of the $p$-adic Abel-Jacobi map implies

$$
0 \neq \log _{\omega_{f}} P\left(\chi_{0}\right)=\log _{\Phi_{f, \chi_{0}}^{*} \omega_{A_{f}}} P\left(\chi_{0}\right)=\log _{\omega_{A_{f}}} \Phi_{f, \chi_{0}}\left(P\left(\chi_{0}\right)\right)=\log _{\omega_{A_{f}}} P_{f}\left(\chi_{0}\right),
$$

and so $P_{f}\left(\chi_{0}\right) \in A_{f, E_{f, \chi}}(F)^{\chi_{0}}$ is nontrivial. Suppose $\chi_{0}=1$. If $E_{f}=\mathbb{Q}$, then $A_{f}$ is an elliptic curve, and $P_{f}(1) \in A_{f, \mathbb{Q}}(K)$ is nontrivial. By Kolyvagin's theorem [1990], we have $\operatorname{rank}_{\mathbb{Z}} A_{f}(K)=1$.

2.3. Main results. In the case where $f \in S_{k}\left(\Gamma_{0}(N), \varepsilon_{f}\right)$ is a normalized newform with partial Eisenstein descent (see Definition 31), i.e., a newform whose Fourier coefficients are congruent to those of an Eisenstein series except possibly at the constant term, we will establish a congruence between Bertolini, Darmon, and Prasanna's anticyclotomic $p$-adic $L$-function and the Katz two-variable $p$-adic $L$-function which holds wherever the former 1-variable $p$-adic $L$-function is defined. A more precise statement is given in our main theorem below. Fix the following assumptions.

Assumptions 1. (1) Let $f \in S_{k}\left(\Gamma_{0}(N), \varepsilon_{f}\right)$ be a normalized newform of weight $k \geq 2$ and level $N>4$.

(2) Let $p \nmid N$ be a rational prime.

(3) Let $K / \mathbb{Q}$ be an imaginary quadratic extension with odd fundamental discriminant $D_{K}<-4$ and $p$ split in $K$.

(4) (Heegner hypothesis) For any prime $\ell \mid N, \ell$ is split in $K / \mathbb{Q}$.

Note that assumption (4) guarantees the existence of an integral ideal $\mathfrak{N} \mid N$ of $\mathcal{O}_{K}$ with

$$
\mathcal{O}_{K} / \mathfrak{N}=\mathbb{Z} / N .
$$

Fix such an $\mathfrak{N}$ and write $\mathfrak{N}=\prod_{\ell \mid N} v$ as a product of primes $v$ of $\mathcal{O}_{K}$ with $v \mid \ell$ for rational primes $\ell \mid N$.

Remark 2. The assumption that $D_{K}<-4$ is odd is made for calculational convenience in [Bertolini et al. 2013, Remark 4.7] and can most likely be removed. See Liu, Zhang, and Zhang's recent generalization of Bertolini, Darmon, and Prasanna's $L$-function and their $p$-adic Waldspurger formula [Liu et al. 2014].

Note that our fixed embedding $i_{p}: \overline{\mathbb{Q}} \hookrightarrow \mathbb{C}_{p}$ determines a prime ideal $\mathfrak{p}$ of $\mathcal{O}_{K}$ above $p$. Let $\mathcal{L}_{p}(f, \chi)$ denote Bertolini, Darmon, and Prasanna's anticyclotomic $p$-adic $L$-function described in Section 3.4, and let $L_{p}(\chi, 0)$ denote the Katz $p$-adic $L$-function described in Section 3.5. Let $F^{\prime}$ and $H_{\mathfrak{N}}$ be the fields defined in Section 3.4, and let $\mathfrak{p}^{\prime}$ be the prime ideal of $\mathcal{O}_{F^{\prime}}$ above $\mathfrak{p}$ determined by $i_{p}$.

Theorem 3 (main theorem). Let $(f, p, K)$ be as in Assumptions 1. Suppose $f$ has Eisenstein descent of type $\left(\psi_{1}, \psi_{2}, N_{+}, N_{-}, N_{0}\right) \bmod \mathfrak{m}$ (see Definition 31) for 
some integral ideal $\mathfrak{m}$ of the ring of integers of a $p$-adic local field $M$ containing $E_{f}$, and suppose $\psi_{1}$ and $\psi_{2}$ are Dirichlet characters over $\mathbb{Q}$ with $\psi_{1} \psi_{2}=\varepsilon_{f}$. Let $\mathfrak{t}$ be an integral ideal of $\mathcal{O}_{K}$ with $\mathcal{O}_{K} / \mathfrak{t}=\mathbb{Z} / \mathfrak{f}\left(\psi_{2}\right)$ and $\mathfrak{t} \mid \mathfrak{N}$. For all $\chi \in \hat{\Sigma}_{\mathrm{cc}}(\mathfrak{N})$ (see Section 3.4 for a precise definition), we have the congruence $\mathcal{L}_{p}(f, \chi) \equiv \psi_{1}^{-1}\left(D_{K}\right)\left(\frac{\left|\mathfrak{f}\left(\psi_{2}\right)\right|^{k} \chi^{-1}(\overline{\mathfrak{t}})}{4 \mathfrak{g}\left(\psi_{2}^{-1}\right)(2 \pi i)^{k+2 j}} \cdot \Xi \cdot L_{p}\left(\psi_{1 / K} \chi^{-1}, 0\right)\right)^{2}\left(\bmod \mathfrak{m} \mathcal{O}_{F_{\mathfrak{p}^{\prime}}, M}\right)$, where $k+2 j \in \mathbb{Z} /(p-1) \times \mathbb{Z}_{p}$ is the signature of $\chi \in \hat{\Sigma}_{\mathrm{cc}}(\mathfrak{N})$ (see Definition 23) and $\begin{aligned} \Xi=\prod_{\ell \mid N_{+}}\left(1-\left(\psi_{2 / K} \chi^{-1}\right)(\bar{v}) \ell^{k-1}\right) & \prod_{\ell \mid N_{-}}\left(1-\left(\psi_{1 / K} \chi^{-1}\right)(\bar{v})\right) \\ & \times \prod_{\ell \mid N_{0}}\left(1-\left(\psi_{2 / K} \chi^{-1}\right)(\bar{v}) \ell^{k-1}\right)\left(1-\left(\psi_{1 / K} \chi^{-1}\right)(\bar{v})\right) .\end{aligned}$

Invoking Bertolini, Darmon, and Prasanna's $p$-adic Waldspurger formula, given as Theorem 25, we can identify the left-hand side of Theorem 3 with a $p$-adic Abel-Jacobi image of a generalized Heegner cycle, and thus derive the following congruence.

Corollary 4. Suppose $\chi \in \Sigma_{\mathrm{cc}}^{(1)}(\mathfrak{N})$ (see Section 3.4 for a precise definition) with infinity type $(k-1-j, 1+j)$, where $0 \leq j \leq k-2$. In the setting of Theorem 3 , we have, for $0 \leq j \leq k-2$,

$$
\begin{array}{r}
\Omega_{p}^{2(k-2-2 j)}\left(\frac{1-\chi^{-1}(\overline{\mathfrak{p}}) a_{p}(f)+\chi^{-2}(\overline{\mathfrak{p}}) \varepsilon_{f}(p) p^{k-1}}{\Gamma(j+1)}\right)^{2}\left(\mathrm{AJ}_{F_{\mathfrak{p}^{\prime}}}\left(\Delta\left(\chi \mathbb{N}_{K}\right)\right)\left(\omega_{f} \wedge \omega_{A}^{j} \eta_{A}^{k-2-j}\right)\right)^{2} \\
\equiv \psi_{1}^{-1}\left(D_{K}\right)\left(\frac{\left|\mathfrak{f}\left(\psi_{2}\right)\right|^{k} \chi^{-1}(\overline{\mathfrak{t}})}{4 \mathfrak{g}\left(\psi_{2}^{-1}\right)(2 \pi i)^{k-2-2 j}} \cdot \Xi \cdot L_{p}\left(\psi_{1 / K} \chi^{-1}, 0\right)\right)^{2}\left(\bmod \mathfrak{m} \mathcal{O}_{F_{\mathfrak{p}^{\prime}} M}\right)
\end{array}
$$

Here $\Omega_{p}$ is the p-adic period defined in Section 3.4, and

$$
\Delta\left(\chi \mathbb{N}_{K}\right):=\sum_{[\mathfrak{a}] \in \operatorname{Cl}\left(\mathcal{O}_{K}\right)}\left(\chi \mathbb{N}_{K}\right)^{-1}(\mathfrak{a}) \cdot \Delta_{\varphi_{\mathfrak{a}} \varphi_{0}} \in \mathrm{CH}^{k-1}\left(X_{k-2}\right)_{0, E_{\chi}}\left(H_{\mathfrak{N}}\right),
$$

where $\Delta_{\varphi_{\mathrm{a}} \varphi_{0}}$ is defined as in Section 3.4.

Remark 5. Corollary 4 can be viewed as providing a new method for verifying a consequence of the Beilinson-Bloch conjecture. In light of the discussion in Section 2.2, we note that for $\chi \in \Sigma_{\mathrm{cc}}^{(1)}(\mathfrak{N})$ we have $L\left(\pi_{f} \times \pi_{\chi^{-1}}, \frac{1}{2}\right)=0$, which implies $L\left(\pi_{f} \times \pi_{\left(\chi^{-1}\right)_{/ F}}, \frac{1}{2}\right)=0$ for any $F / H_{\mathfrak{N}}$ in situation $(S)$. Hence, the BeilinsonBloch conjecture predicts that $\operatorname{dim}_{E_{f, \chi}} \epsilon_{(f, \chi)_{/ F}} \mathrm{CH}^{k-1}\left(X_{k-2}\right)(F) \geq 1$. Corollary 4 shows that, in the setting where $f$ has Eisenstein descent $\bmod \mathfrak{m}$, if

$$
\Xi \cdot L_{p}\left(\psi_{1 / K} \chi^{-1}, 0\right) \not \equiv 0\left(\bmod \mathfrak{m} \mathcal{O}_{F_{p^{\prime}}^{\prime}}\right)
$$


then the generalized Heegner cycle $\Delta\left(\chi \mathbb{N}_{K}\right)$ induces the ostensibly nontrivial algebraic cycle class. We are thus reduced to verifying the $p$-nondivisibility of the above special values of the Katz $p$-adic $L$-function. In Theorem 7 we explicitly evaluate Katz $L$-values corresponding to classical Heegner cycles (see Remark 8) using a theorem of Gross in order to find explicit non- $p$-divisibility criteria. One might expect to be able to carry out studies of $p$-nondivisibility for more general Katz $L$-values in order to establish similar explicit nontriviality criteria for more general Heegner cycles, but we do not do this here.

Suppose that $\varepsilon_{f}=1$ and that $k$ is even. Note that the anticyclotomic line intersects with the cyclotomic line when $j=k / 2-1$, in the notation of Corollary 4. For this $j$, we can take the particular character $\chi=\mathbb{N}_{K}^{-k / 2} \in \Sigma_{\mathrm{cc}}^{(1)}(\mathfrak{N})$. Applying Gross's factorization (Theorem 28) of the Katz $L$-function on the cyclotomic line to the righthand side of Theorem 3, we get the following explicit congruences between special values of $p$-adic $L$-functions. First, fix the following notation for convenience.

Definition 6. Suppose we are given an imaginary quadratic field $K$. For any Dirichlet character $\psi$ over $\mathbb{Q}$, let

$$
\psi_{0}:= \begin{cases}\psi & \text { if } \psi \text { even } \\ \psi \varepsilon_{K} & \text { if } \psi \text { odd }\end{cases}
$$

Theorem 7. In the setting of Theorem 3, specializing to $\varepsilon_{f}=1, k$ even, and $\psi_{1}=\psi_{2}^{-1}=\psi$,

$$
\left(\frac{p^{k / 2}-a_{p}(f)+p^{k / 2-1}}{p^{k / 2} \Gamma\left(\frac{k}{2}\right)}\right)^{2} \mathrm{AJ}_{F_{\mathfrak{p}^{\prime}}}\left(\Delta\left(\mathbb{N}_{K}^{1-k / 2}\right)\right)^{2}\left(\omega_{f} \wedge \omega_{A}^{k / 2-1} \eta_{A}^{k / 2-1}\right)
$$

is congruent to

$$
\frac{\Xi^{2}}{4}\left(\frac{1}{k}\left(1-\psi^{-1}(p) p^{k / 2-1}\right) B_{\frac{k}{2}, \psi_{0}^{-1} \varepsilon_{K}^{k / 2}} \cdot L_{p}\left(\psi_{0}\left(\varepsilon_{K} \omega\right)^{1-k / 2}, \frac{k}{2}\right)\right)^{2}\left(\bmod \mathfrak{m} \mathcal{O}_{F_{p^{\prime}}^{\prime} M}\right)
$$

if $\psi_{0}\left(\varepsilon_{K} \omega\right)^{1-k / 2} \neq 1$ or $k>2$, and

$$
\frac{\Xi^{2}}{4}\left(\frac{p-1}{p} \log _{p} \bar{\alpha}\right)^{2}\left(\bmod \mathfrak{m} \mathcal{O}_{F_{p^{\prime}}^{\prime} M}\right)
$$

otherwise.

If $\mathfrak{m}=\lambda$, where $\lambda$ is some prime ideal of $\mathcal{O}_{M}$ above $p$, then more explicitly

$$
\left(\frac{p^{k / 2}-a_{p}(f)+p^{k / 2-1}}{p^{k / 2} \Gamma\left(\frac{k}{2}\right)}\right)^{2} \mathrm{AJ}_{F_{\mathfrak{p}^{\prime}}}\left(\Delta_{f}\left(\mathbb{N}_{K}^{1-k / 2}\right)\right)^{2}\left(\omega_{f} \wedge \omega_{A}^{k / 2-1} \eta_{A}^{k / 2-1}\right)
$$


is congruent to

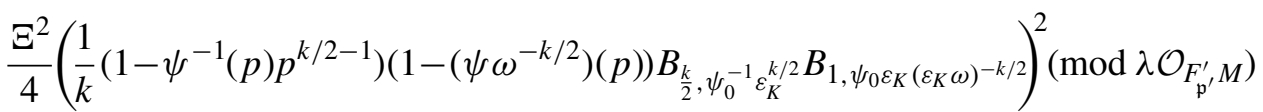
if $\psi_{0}\left(\varepsilon_{K} \omega\right)^{1-k / 2} \neq 1$,

$$
\frac{\Xi^{2}}{4}\left(\frac{1}{k}\left(1-\psi^{-1}(p) p^{k / 2-1}\right) B_{\frac{k}{2}, \psi_{0}^{-1} \varepsilon_{K}^{k / 2}} \cdot L_{p}\left(1, \frac{k}{2}\right)\right)^{2}\left(\bmod \lambda \mathcal{O}_{F_{\mathfrak{p}^{\prime}}^{\prime} M}\right)
$$

if $\psi_{0}\left(\varepsilon_{K} \omega\right)^{1-k / 2}=1, k>2$, and

$$
\frac{\Xi^{2}}{4}\left(\frac{p-1}{p} \log _{p} \bar{\alpha}\right)^{2}\left(\bmod \lambda \mathcal{O}_{F_{\mathfrak{p}^{\prime}}^{\prime} M}\right)
$$

otherwise.

Here

$$
\Delta\left(\mathbb{N}_{K}^{1-k / 2}\right):=\sum_{[\mathfrak{a}] \in \operatorname{Cl}\left(\mathcal{O}_{K}\right)} \mathbb{N}_{K}^{k / 2-1}(\mathfrak{a}) \cdot \Delta_{\varphi_{\mathfrak{a}} \varphi_{0}} \in \mathrm{CH}^{k-1}\left(X_{k-2}\right)_{0, \mathbb{Q}}\left(H_{\mathfrak{N}}\right),
$$

where $\Delta_{\varphi_{\mathfrak{a}} \varphi_{0}}$ is defined as in Section 3.4,

$$
\Xi=\prod_{\ell \mid N_{+}}\left(1-\frac{\psi^{-1}(\ell)}{\ell^{1-k / 2}}\right) \prod_{\ell \mid N_{-}}\left(1-\frac{\psi(\ell)}{\ell^{k / 2}}\right) \prod_{\ell \mid N_{0}}\left(1-\frac{\psi^{-1}(\ell)}{\ell^{1-k / 2}}\right)\left(1-\frac{\psi(\ell)}{\ell^{k / 2}}\right),
$$

$\log _{p}$ is the Iwasawa $p$-adic logarithm (i.e., with branch $\log _{p} p=0$ ), and $\bar{\alpha} \in \mathcal{O}_{K}$ such that $(\bar{\alpha})=\overline{\mathfrak{p}}^{h_{K}}$.

Remark 8. In the setting of Theorem 7, the generalized Heegner cycle $\Delta\left(\mathbb{N}_{K}^{1-k / 2}\right)$ can be mapped via a correspondence to a classical Heegner cycle arising on the classical Kuga-Sato variety $W_{k-2}$ defined in Section 2.2; see, for example, [Bertolini et al. 2015, Proposition 4.1.1, Theorem 4.1.3]. Therefore, in view of Remark 5, Theorem 7 gives an explicit congruence criterion for showing the nontriviality of a classical Heegner cycle class and thus verifying the aforementioned consequence of the Beilinson-Bloch conjecture.

Remark 9. For any $x \in \overline{\mathfrak{p}}$, we have $\operatorname{ord}_{p}(x)=0$, so we can write $x=a+2 \pi$ for $\pi \in \mathfrak{p} \mathcal{O}_{K_{\mathfrak{p}}}$ and $a \in \mathcal{O}_{K_{\mathfrak{p}}}^{\times}$. Then $x^{h_{K}}=a^{h_{K}}+h_{K} a^{h_{K}-1} 2 \pi+\cdots$, so

$$
\operatorname{ord}_{p}\left(\frac{p-1}{p} \log _{p} \bar{\alpha}\right)=\operatorname{ord}_{p}\left(2 h_{K}\right)
$$

Remark 10. In certain situations, one can use explicit special value formulas to further evaluate the right-hand side of the congruences in Theorem 7. For example, 
when $k=2$ and $\psi_{0} \neq 1$, by Leopoldt's formula and the fact that $\varepsilon_{K}(p)=1$ since $p$ is split in $K$, we have

$$
L_{p}\left(\psi_{0}, 1\right)=\left(1-\frac{\psi(p)}{p}\right) \frac{\mathfrak{g}\left(\psi_{0}\right)}{\left|\mathfrak{f}\left(\psi_{0}\right)\right|} \sum_{a=1}^{\left|\mathfrak{f}\left(\psi_{0}\right)\right|} \psi_{0}^{-1}(a) \log _{p}\left(1-\exp \left(\frac{2 \pi i a}{\left|\mathfrak{f}\left(\psi_{0}\right)\right|}\right)\right) .
$$

Furthermore, Diamond computes explicit formulas for the values of $L_{p}(\chi, n)$ when $n$ is a positive integer; see [Diamond 1979].

Definition 11. Attached to any normalized newform $f \in S_{k}\left(\Gamma_{0}(N), \varepsilon_{f}\right)$ and prime $\lambda$ above $p$ of a number field $M$ containing $E_{f}$ is a unique semisimple $\lambda$-adic Galois representation $\rho_{f}: \operatorname{Gal}(\overline{\mathbb{Q}} / \mathbb{Q}) \rightarrow \mathrm{GL}_{2}\left(M_{\lambda}\right)$ (see Section 3.6). Choosing a $\operatorname{Gal}(\overline{\mathbb{Q}} / \mathbb{Q})$-invariant lattice in $M_{\lambda}^{2}$, one obtains an associated semisimple mod- $\lambda$ Galois representation $\bar{\rho}_{f}$ (which turns out to be independent of the choice of lattice). If $p \nmid N$ and if $\bar{\rho}_{f}$ is reducible, we will see (in Theorem 34) that $N=N_{+} N_{-} N_{0}$, where $N_{+} N_{-}$is squarefree and $N_{0}$ is squarefull, such that there are Dirichlet characters $\psi_{1}$ and $\psi_{2}$ over $\mathbb{Q}$ with $\psi_{1} \psi_{2}=\varepsilon_{f}$, and where $\ell \mid N_{+}$implies $a_{\ell} \equiv \psi_{1}(\ell)(\bmod \lambda)$, $\ell \mid N_{-}$implies $a_{\ell} \equiv \psi_{2}(\ell) \ell^{k-1}(\bmod \lambda)$, and $\ell \mid N_{0}$ implies $a_{\ell} \equiv 0(\bmod \lambda)$. We then say that $\bar{\rho}_{f}$ is reducible of type $\left(\psi_{1}, \psi_{2}, N_{+}, N_{-}, N_{0}\right)$.

By Theorem 34, we immediately get the following corollary.

Corollary 12. Let $(f, p, K)$ be as in Assumptions 1 , and suppose $\bar{\rho}_{f}$ is reducible of type $\left(\psi_{1}, \psi_{2}, N_{+}, N_{-}, N_{0}\right)$, where $\bar{\rho}_{f}$ is the semisimple mod- $\lambda$ residual representation of $\rho_{f}$ for some prime ideal $\lambda$ of the ring of integers of a $p$-adic local field $M$ containing $E_{f}$. Then the conclusions of Theorems 3 and 7 hold with $\mathfrak{m}=\lambda$.

Applying Theorem 7 to the case when $k$ is 2 and $f$ is defined over $\mathbb{Q}$, and is thus associated with an elliptic curve $E / \mathbb{Q}$ of conductor $N$, we can show the Heegner point $P_{E}(K)$ is nontorsion when the primes dividing $p N$ satisfy certain congruence conditions and $p$ does not divide certain Bernoulli numbers. First, decompose the conductor $N=N_{\text {split }} N_{\text {nonsplit }} N_{\text {add }}$ into factors such that $\ell \mid N_{\text {split }}$ implies $\ell$ is of split multiplicative reduction, $\ell \mid N_{\text {nonsplit }}$ implies $\ell$ is of nonsplit multiplicative reduction, and $\ell \mid N_{\text {add }}$ implies $\ell$ is of additive reduction.

Theorem 13. Suppose $E / \mathbb{Q}$ is any elliptic curve of conductor $N$ with reducible mod-p Galois representation $E[p]$, or equivalently, $E[p]^{\mathrm{ss}} \cong \mathbb{F}_{p}(\psi) \oplus \mathbb{F}_{p}\left(\psi^{-1} \omega\right)$, where $p>2$ is a prime of good reduction for $E$ and $\psi$ is some Dirichlet character with $\mathfrak{f}(\psi)^{2} \mid N_{\text {add. }}$. Suppose further that

(1) $\psi(p) \neq 1$,

(2) $N_{\text {split }}=1$ (i.e., E has no primes of split multiplicative reduction),

(3) $\ell \mid N_{\text {add }}$ implies either $\psi(\ell) \neq 1$ and $\ell \not \equiv-1(\bmod p)$, or $\psi(\ell)=0$. 
Then, for any imaginary quadratic field $K$ in which $p$ splits, such that $K$ satisfies the Heegner hypothesis with respect to $E$, and $p \nmid B_{1, \psi_{0}^{-1} \varepsilon_{K}} B_{1, \psi_{0} \omega^{-1}}$, the associated Heegner point $P_{E}(K) \in E(K)$ (see Section 3.4) is nontorsion. In particular, $\operatorname{rank}_{\mathbb{Z}} E(K)=1$.

Remark 14. When $\psi$ is quadratic, the condition $p \nmid B_{1, \psi_{0} \varepsilon_{K}}$ of Theorem 13 is equivalent to $p \nmid h_{K_{\psi_{0}} \cdot K}$. (This follows because $B_{1, \psi_{0}^{-1} \varepsilon_{K}}=-2 h_{K_{\psi_{0}} \cdot K} /\left|\mathcal{O}_{K_{\psi_{0}} \cdot K}^{\times}\right|$by the functional equation and analytic class number formula.)

Remark 15. In light of Theorems 34 and 35, which imply that $a_{\ell}(E) \equiv \psi(\ell)$ or $\psi^{-1}(\ell) \ell(\bmod p)$ for $\ell \| N$ (i.e., $\left.\ell \mid N_{\text {split }} N_{\text {nonsplit }}\right)$ when $E[p]^{\mathrm{ss}} \cong \mathbb{F}_{p}(\psi) \oplus \mathbb{F}_{p}\left(\psi^{-1} \omega\right)$, condition (2) can be phrased as

$(2)^{\prime}$ for every $\ell \| N, \psi(\ell) \equiv-1$ or $-\ell(\bmod p)$.

In terms of local root numbers $w_{\ell}(E)$, since $w_{\ell}(E)=-a_{\ell}(E)$ for $\ell \| N$, conditions (2) and (2) can also be viewed as requiring the corresponding local root numbers to all be +1 .

Remark 16. Theorem 13 (at least partially) recovers a much earlier result of Mazur [1979, Theorem, p. 231], which considers the case $N=$ prime $\neq p$. In Mazur's setting, we suppose that $E[p]^{\mathrm{ss}} \cong \mathbb{F}_{p} \oplus \mathbb{F}_{p}(\omega)$, where $N=\ell \neq p$, let $\psi$ be an even quadratic character and choose an imaginary quadratic field $K$. (In Mazur's notation, we are taking $\chi=\psi \varepsilon_{K}$ and $K_{\chi}=K_{\psi} \cdot K=K_{\psi \varepsilon_{K}}$.) The case of Mazur's theorem we recover is as follows.

Suppose $(E, p, \psi, K)$ as above are such that

(1) $K$ satisfies the Heegner hypothesis with respect to $E \otimes \psi$,

(2) $p$ splits in $K$ and $D_{K}<-4$,

(3) $\psi(p)=-1$,

(4) $p \nmid B_{1, \psi \omega^{-1}}$,

(5) $\operatorname{lcm}\left(\ell,|\mathfrak{f}(\psi)|^{2}\right)>4, \psi(\ell) \neq 1$ and $p \nmid h_{K_{\psi} \cdot K}$.

Then $\operatorname{rank}_{\mathbb{Z}}\left(E \otimes \psi \varepsilon_{K}\right)(\mathbb{Q})=0$.

This follows from Theorem 13 since the latter implies $\operatorname{rank}_{\mathbb{Z}}(E \otimes \psi)(K)=1$, and then $\operatorname{rank}_{\mathbb{Z}}\left(E \otimes \psi \varepsilon_{K}\right)(\mathbb{Q})=0$ follows from root number considerations (see Proposition 50).

Assumptions (1)-(4) above are extraneous in the full generality of Mazur's theorem, with (5) being the pertinent hypothesis. These assumptions have the following effects: (1) is part (4) of Assumptions 1 and guarantees that $L((E \otimes \psi) / K, 1)=0$; (2) is part (3) of Assumptions 1; (3) excludes the possibility of a "trivial zero" from the Kubota-Leopoldt factor $L_{p}\left(\psi \varepsilon_{K} \omega, 0\right)$, which shows up in the congruence of Theorem 7; (4) essentially controls the Selmer group $\operatorname{Sel}_{p}((E \otimes \psi) / \mathbb{Q}$ ) (see Remark 45) so that $\operatorname{rank}_{\mathbb{Z}}(E \otimes \psi)(\mathbb{Q}) \leq 1$. Mazur's original statement also allows 
for $p \mid \mathfrak{f}(\psi)$, which is ruled out by part (2) of Assumptions 1. We should note, however, that Mazur requires the additional assumptions

(6) $\ell \geq 11$,

(7) $p$ divides the numerator of $\left(\frac{\ell-1}{12}\right)$,

(8) $\left(p,\left|\mathfrak{f}\left(\psi \varepsilon_{K}\right)\right|\right) \neq(3,3)$.

Assumption (6) is a technical assumption and is stronger than our assumption $\operatorname{lcm}\left(\ell,|\mathfrak{f}(\psi)|^{2}\right)>4$ on the level of $E \otimes \psi$, which in turn originates from part (1) of Assumptions 1; (7) means that the newform $f_{E} \in S_{2}\left(\Gamma_{0}(\ell)\right)$ of $E$ has full Eisenstein descent (see Definition 31), and is automatically satisfied when $E[p]$ is reducible and $p>3$ (see Remark 33); (8) is taken care of by our assumption $D_{K}<-4$ from part (3) of Assumptions 1.

Perhaps more interesting is the connection between Theorem 13 and the "supplement" to Mazur's theorem, which states that a Heegner point is nontorsion when " $\psi(\ell) \neq 1$ " in Assumption (5) above is replaced with " $\psi(\ell)=1$ " (see [Mazur 1979, Theorem, p. 237]). In particular, $E$ satisfies the Heegner hypothesis with respect to both $K$ and $K_{\psi} \cdot K$, and $E \otimes \psi \varepsilon_{K}$ has root number -1 so that $P_{E \otimes \psi}(K) \in\left(E \otimes \psi \varepsilon_{K}\right)(\mathbb{Q})$. As is pointed out in [Mazur 1979], under this assumption, the Kubota-Leopoldt factor $L_{p}\left(\psi^{-1} \varepsilon_{K} \omega, 0\right)$ from Theorem 7 can be related $\bmod p$ to $(1 / p) \frac{d}{d s} L\left(E, \psi \varepsilon_{K}, s\right)_{\mid s=1}$, itself being related to the $p$-adic height $\bmod p$ of

$$
P_{E}\left(K_{\psi} \cdot K\right)^{-}:=P_{E}\left(K_{\psi} \cdot K\right)-\overline{P_{E}\left(K_{\psi} \cdot K\right)} \in\left(E \otimes \psi \varepsilon_{K}\right)(\mathbb{Q}) .
$$

Note that $\psi(\ell)=1$ places $E \otimes \psi$ outside the scope of Theorem 13, but perhaps this descent result in tandem with Mazur's observation and the mod- $p$ factorization of Theorem 7 suggests an identity (in certain cases) relating the formal logarithm of $P_{E \otimes \psi}(K) \in\left(E \otimes \psi \varepsilon_{K}\right)(\mathbb{Q})$ with the $p$-adic height of $P_{E}\left(K_{\psi} \cdot K\right)^{-} \in\left(E \otimes \psi \varepsilon_{K}\right)(\mathbb{Q})$ times another quantity, perhaps related to the order of $\amalg(E \otimes \psi / \mathbb{Q})\left[p^{\infty}\right]$. A deeper comparison between the results of this paper and Mazur's would be an interesting direction for further investigation.

Remark 17. One of the referees has also pointed out an analogy between Mazur's result and Theorem 13 and the two "reciprocity laws" which Bertolini and Darmon [2005] established in their work on the anticyclotomic Iwasawa main conjecture for elliptic curves. Let $E / \mathbb{Q}$ be an elliptic curve with newform $f_{E}$. Mazur's descent result assumes $E \otimes \varepsilon_{K}$ has root number -1 over $\mathbb{Q}$ and computes the image of $P_{E}(K)^{-}$inside the $p$-primary part of the component group of the special fiber of a Néron model of $E$ over $\mathbb{Z}_{N}$. Using an Eisenstein congruence at $p$, Mazur shows that nonvanishing of this image is equivalent to $p \nmid h_{K}$, i.e., the $p$-nondivisibility of a special value of a certain Kubota-Leopoldt $p$-adic $L$-function. Bertolini and 
Darmon's first reciprocity law also assumes $E$ has root number -1 over $K$ and computes the image inside the $p$-primary part of the component group of the special fiber of a Néron model over $\mathbb{Z}_{\ell^{2}}$ of some abelian variety (arising as a quotient of $J_{1}(N \ell)$ for some prime $\ell \nmid N$ which is inert in $K$ ). (Here $\mathbb{Z}_{\ell^{2}}$ is the unramified quadratic extension of $\mathbb{Z}_{\ell}$.) Using a level-raising congruence modulo some power of $p$ of $f_{E}$ with an eigenform $g$ of level $N \ell$ (so that $g$ has root number +1 over $K$ ), Bertolini and Darmon equate this image (using a Waldspurger-type formula) with a special value of an anticyclotomic $p$-adic $L$-function attached to $g$.

In Theorem 7 applied to $E$, we assume that $E$ has root number -1 over $K$ and computes the $p$-adic formal logarithm of $P_{E}(K)$ modulo some power of $p$. We then use an Eisenstein congruence at $p$ to equate this with a special value of a Katz $p$-adic $L$-function. Bertolini and Darmon's second reciprocity law similarly assumes $E$ has root number -1 over $K$ and computes the image of $P_{E}(K)$ inside the finite part of some power-of-p-(Bloch-Kato) Selmer group of $E$. The formal logarithm at $p$ modulo this same power of $p$ in particular maps into this Selmer group. Using another level-raising congruence to an eigenform $g$ of level $N \ell_{1} \ell_{2}$ for distinct "admissible" primes $\ell_{1}, \ell_{2} \nmid N$, Bertolini and Darmon again equate this image with a special value of an anticyclotomic $p$-adic $L$-function of $g$. It should be noted that the $p$-adic Waldspurger formula of Bertolini, Darmon, and Prasanna (Theorem 25) is itself established through exploiting yet another "sign change" congruence, namely by considering congruences with a newform $f$ and its various anticyclotomic twists, considering these twists in a $p$-adic family, varying the twists through the interpolation region where the global root number is +1 , and taking a $p$-adic limit into a region where the root number is -1 .

Let us return to the setting of Theorem 13. Considering quadratic twists of $E$ when $E[p]^{\mathrm{ss}} \cong \mathbb{F}_{p}(\psi) \oplus \mathbb{F}_{p}\left(\psi^{-1} \omega\right)$ (which amounts to varying $\psi$ through quadratic characters), we obtain congruence criteria for the $\mathfrak{f}(\psi)$ which, when satisfied, imply $\operatorname{rank}_{\mathbb{Z}}(E \otimes \psi)(K)=1$. In Section 5.5, we consider $p=3$ and use quadratic class number 3-divisibility results to show the following.

Corollary 18. Suppose $E / \mathbb{Q}$ has reducible mod-3 Galois representation $E[3]$. Then we have:

(1) If $E$ is reducible of type ( $\left.\psi, \psi^{-1}, N_{+}, N_{-}, N_{0}\right)$ for some quadratic character $\psi$, then a positive proportion of quadratic twists of $E$, ordered by absolute value of discriminant, have rank 0 or 1 .

(2) If $E$ is semistable, then a positive proportion of real quadratic twists of E, ordered by absolute value of discriminant, have rank 1 and a positive proportion of imaginary quadratic twists of $E$, likewise ordered, have rank 0 .

Remark 19. For $E$ as in the statement of Corollary 18, explicit lower bounds for these proportions are given in the statement of Theorems 53 and 54, respectively. 
One can also use congruence criteria provided by Theorem 13 to find explicit examples of quadratic twists $E \otimes \psi$ with $\operatorname{rank}_{\mathbb{Z}}(E \otimes \psi)(K)=1$.

Remark 20. Recent work of Harron and Snowden [2015] in particular shows that

$$
\lim _{X \rightarrow \infty} \frac{N_{\mathbb{Z} / 3}(X)}{X^{1 / 3}} \approx 1.5221
$$

where $N_{G}(X)$ denotes the number of (isomorphism classes of) elliptic curves $E$ over $\mathbb{Q}$ up to (naive) height $X$ with $E(\mathbb{Q})^{\text {tor }} \cong G$. Corollary 18 applies to all curves with nontrivial rational 3-torsion, and so applies to at least a number of curves on the order of $X^{1 / 3}$ up to height $X$.

The organization of this paper is as follows. In Section 3, we review some preliminaries, including definitions pertinent to our discussion and a review of algebraic and $p$-adic modular forms. In Section 3.4, we recall Bertolini, Darmon, and Prasanna's anticyclotomic $p$-adic $L$-function as well as their $p$-adic Waldspurger formula, which gives the value of the image of a certain generalized Heegner cycle under the $p$-adic Abel-Jacobi in terms of a special value of their $p$-adic $L$-function. In Section 3.5, we recall Katz's $p$-adic $L$-function attached to Hecke characters over imaginary quadratic fields $K$ as well as recall Gross's factorization of the $L$-function on the cyclotomic line. In Section 3.6, we define the notion of a normalized newform $f$ having Eisenstein descent, and relate it to the reducibility of the residual $p$-adic Galois representation of $f$.

In Section 4, we prove our main congruence by showing that certain twisted traces over CM points of Maass-Shimura derivatives $\partial^{j}$ of Eisenstein series represent special values of complex $L$-functions of Hecke characters. The twisted traces considered in [Bertolini et al. 2013] are exactly of the same kind, with the sole difference being that the Eisenstein series is replaced by a cusp form. Interpolating these twisted traces yields the Katz $p$-adic $L$-function and Bertolini, Darmon, and Prasanna's anticyclotomic $p$-adic $L$-functions, respectively. When $f$ has partial Eisenstein descent with associated Eisenstein series $G$, we use the corresponding congruence between the twisted traces of $\partial^{j} f$ and $\partial^{j} G$ to derive the congruence between $p$-adic $L$-functions.

In Section 5, we apply our congruence formula to the problem of finding nontrivial algebraic cycles whose existence is predicted by the Beilinson-Bloch conjecture. We also give examples of Eisenstein descent, including ways to construct such newforms (see Constructions 38 and 40), and applications of our main theorem to showing nontriviality of algebraic cycle classes. In Section 5.4, we address the particular case of showing nontriviality of generalized Heegner cycles attached to quadratic twists of the Ramanujan $\Delta$ function. In Section 5.5, we examine the case of elliptic curves and derive an explicit criterion to show $\operatorname{rank}_{\mathbb{Z}}(E(K))=1$ for elliptic curves $E / \mathbb{Q}$ with reducible mod- $p$ Galois representation. 
In Section 5.6, we show that when $E$ has reducible mod-3 Galois representation, a positive proportion of quadratic twists of $E$ have rank 0 or 1 . These results in certain cases extend those of [Vatsal 1999], who exhibited an infinite family of elliptic curves (namely, those which are semistable with rational 3-torsion), for each member of which a positive proportion of its imaginary quadratic twists have rank 0 . Our result gives a larger infinite family of elliptic curves (namely, those which are semistable with reducible mod-3 Galois representation), for each member of which a positive proportion of its imaginary quadratic twists have rank 0 and a positive proportion of its real quadratic twists have rank 1.

\section{Preliminaries}

In this section, we review some preliminaries relating to Bertolini, Darmon, and Prasanna's and Katz's $p$-adic $L$-functions. Our discussion follows [Bertolini et al. 2013].

\subsection{Algebraic modular forms. Recall}

$$
\begin{aligned}
& \Gamma_{1}(N):=\left\{\gamma \in \mathrm{SL}_{2}(\mathbb{Z}): \gamma \equiv\left(\begin{array}{ll}
1 & * \\
0 & 1
\end{array}\right)(\bmod N)\right\}, \\
& \Gamma_{0}(N):=\left\{\gamma \in \mathrm{SL}_{2}(\mathbb{Z}): \gamma \equiv\left(\begin{array}{ll}
* & * \\
0 & *
\end{array}\right)(\bmod N)\right\},
\end{aligned}
$$

and note that $\Gamma_{1}(N) \subset \Gamma_{0}(N)$. From now on, let $\Gamma$ be $\Gamma_{1}(N)$ or $\Gamma_{0}(N)$. For $i=0,1$, let $Y_{i}(N)$ be the associated modular curve whose complex points are in bijection with the Riemann surface $\Gamma_{i}(N) \backslash \mathcal{H}^{+}$, and which classifies pairs $(E, t)$ consisting of an elliptic curve $E$ and a $\Gamma_{i}(N)$-level structure $t$ on $E$ (so $\mathbb{Z} / N \cong t \subset E[N]$ for $i=0$, and $t \in E[N]$ of exact order $N$ for $i=1)$. Let $X_{i}(N)$ denote the compactification of $Y_{i}(N)$. Let $\pi: \mathcal{E} \rightarrow Y_{1}(N)$ denote the universal elliptic curve with $\Gamma_{1}(N)$-level structure. Throughout the paper, we shall use the interpretation of algebraic modular forms as global sections of powers $\bar{\omega}^{k}$ of the Hodge bundle $\bar{\omega}:=\pi_{*} \Omega_{\mathcal{E} / Y_{1}(N)}^{1}$ of relative differentials $\mathcal{E} / Y_{1}(N)$ (which we may extend to $X_{1}(N)$ when $N>4$ ), so that an algebraic modular form can be thought of as a function on isomorphism classes of triples $[(E, t, \omega)]$ satisfying base-change compatibility and homogeneity conditions; here $E$ is an elliptic curve, $t$ a $\Gamma$-level structure, and $\omega$ an invariant differential on $E$. For details, see [Bertolini et al. 2013, Section 1.1]. Denote by

$$
S_{k}(\Gamma, R) \subset M_{k}(\Gamma, R) \subset M_{k}^{*}(\Gamma, R)
$$

the space of weight- $k$ algebraic $\Gamma$-cuspforms over a ring $R$, the space of weight- $k$ algebraic $\Gamma$-modular forms, and the space of weight $k$ weakly holomorphic $\Gamma$-modular forms, respectively. (See Section 1.1 of loc. cit. for precise definitions.) We have 
similar inclusions for the corresponding spaces considered with nebentypus $\varepsilon$. We will suppress the base ring " $R$ " when it is obvious from context.

Suppose $F \subset \mathbb{C}$ is a field containing the values of a Dirichlet character $\varepsilon$, and suppose $f \in M_{k}^{*}\left(\Gamma_{0}(N), F, \varepsilon\right)$. Then we have an associated holomorphic function on $\mathcal{H}^{+}$given by

$$
f(\tau):=f\left(\mathbb{C} /(\mathbb{Z}+\mathbb{Z} \tau), \frac{1}{N}, 2 \pi i d z\right),
$$

where $d z$ is the standard differential on $\mathbb{C} / \Lambda$ with period lattice equal to $\Lambda$. Note that $f(\tau)$ satisfies the usual weight- $k, \epsilon$-twisted modularity condition for $\Gamma_{0}(N)$,

$$
f(\gamma \cdot \tau)=\varepsilon(d)(c \tau+d)^{k} f(\tau)
$$

for $\gamma=\left(\begin{array}{ll}a & b \\ c & d\end{array}\right) \in \Gamma_{0}(N)$. The function $f(\tau)$ in fact completely determines the weakly holomorphic modular form $f \in M_{k}^{*}\left(\Gamma_{0}(N), F, \varepsilon\right)$.

Denote the space of weight- $k$ nearly holomorphic $\Gamma$-modular forms over $F$ and nebentypus $\varepsilon$ by $N_{k}(\Gamma, F, \varepsilon)$. (See Section 1.2 of loc. cit. for precise definitions.) We have the classical Maass-Shimura operator $\partial_{k}: N_{k}(\Gamma, F, \varepsilon) \rightarrow N_{k+2}(\Gamma, F, \varepsilon)$ acting on nearly holomorphic $\Gamma$-modular forms by the formula

$$
\partial_{k} f(\tau):=\frac{1}{2 \pi i}\left(\frac{d}{d \tau}+\frac{k}{\tau-\bar{\tau}}\right) f(\tau) .
$$

Note that $\partial_{k}$ does not preserve holomorphy, but preserves near-holomorphy. We let $\partial_{k}^{j}:=\partial_{k+2(j-1)} \circ \cdots \circ \partial_{k}: N_{k}(\Gamma, F, \varepsilon) \rightarrow N_{k+2 j}(\Gamma, F, \varepsilon)$, and omit $k$ when it is obvious from context.

3.2. $p$-adic modular forms. Fix a rational prime $p$. We will interpret $p$-adic modular forms analogously to the previous definition of algebraic modular forms, i.e., as global sections of a rigid analytic line bundle over the ordinary locus of $X_{i}(N) / \mathbb{C}_{p}$, or equivalently as functions on isomorphism classes of triples $[(E, t, \omega)]$ defined over $p$-adic rings satisfying certain homogeneity and base-change properties. (For details, see [Bertolini et al. 2013, Section 1.3].)

We have the classical Atkin-Serre operator $\theta: M_{k}^{(p)}(\Gamma, F, \varepsilon) \rightarrow M_{k+2}^{(p)}(\Gamma, F, \varepsilon)$ acting on $p$-adic modular forms, whose effect on $q$-expansions is given by

$$
\theta\left(\sum_{n=0}^{\infty} a_{n} q^{n}\right)=q \frac{d}{d q} \sum_{n=0}^{\infty} a_{n} q^{n}=\sum_{n=1}^{\infty} n a_{n} q^{n} .
$$

Now recall our complex and $p$-adic embeddings $i_{\infty}: F \hookrightarrow \mathbb{C}$ and $i_{p}: F \hookrightarrow \mathbb{C}_{p}$, as well as our field isomorphism $i: \mathbb{C} \stackrel{\sim}{\longrightarrow} \mathbb{C}_{p}$ such that $i_{p}=i \circ i_{\infty}$. Let $K$ be an imaginary quadratic field and let $H$ be the Hilbert class field over $K$. Suppose $E / F$ is a curve with complex multiplication (i.e., such that $\operatorname{End}_{H}(E)$ is isomorphic to an order of $\left.\mathcal{O}_{K}\right)$, and let $(E, t, \omega)$ be a triple defined over $F$. Using $i_{\infty}$ and $i_{p}$, we 
can view $(E, t, \omega)$ as a triple over $\mathbb{C}$ and $\mathbb{C}_{p}$, respectively. Using $i$, we can view $f \in M_{k}^{*}(\Gamma, F, \varepsilon)$ as an element of $M_{k}^{(p)}(\Gamma, F, \varepsilon)$ (after possibly rescaling). The following theorem, due to Shimura and Katz, states that the values of $\partial^{j} f$ and $\theta^{j} f$ coincide on ordinary CM triples.

Theorem 21 (see [Bertolini et al. 2013, Proposition 1.12]). Suppose $(E, t, \omega)$ is a triple defined over $F$, where $E$ has complex multiplication by an order of $\mathcal{O}_{K}$, and assume that $E$, viewed as an elliptic curve over $\mathcal{O}_{\mathbb{C}_{p}}$, is ordinary. Suppose $f \in M_{k}^{*}(\Gamma, F, \varepsilon)$. Then, for $j \geq 0$, we have:

(1) $\partial^{j} f(E, t, \omega) \in i_{\infty}(F)$.

(2) $\theta^{j} f(E, t, \omega) \in i_{p}(F)$.

(3) Viewing these quantities as elements of $F$ (i.e., identifying $i_{\infty}(F) \stackrel{i_{p} \circ i_{\infty}^{-1}}{\longrightarrow} i_{p}(F)$ ), we have

$$
\partial^{j} f(E, t, \omega)=\theta^{j} f(E, t, \omega) .
$$

Denote by b the " $p$-depletion" operator on $M_{k}^{(p)}(\Gamma, F, \varepsilon)$ (see [Bertolini et al. 2013, Section 3.8]), whose action on $q$-expansions $f(q)=\sum_{n=0}^{\infty} a_{n}(f) q^{n}$ is given by

$$
f^{b}(q)=f(q)-a_{p}(f) f\left(q^{p}\right)+\varepsilon(p) p^{k-1} f\left(q^{p^{2}}\right),
$$

so that $a_{n}\left(f^{b}\right)=0$ for all $n>0$ with $(n, p) \neq 1$.

3.3. Isogenies and generalized Heegner cycles. Recall our assumption that the imaginary quadratic field $K$ satisfies the Heegner hypothesis with respect to $N$ :

$$
\text { for all primes } \ell \mid N, \ell \text { is split in } K / \mathbb{Q} \text {. }
$$

This condition implies that there exists an integral ideal $\mathfrak{N}$ of $\mathcal{O}_{K}$ such that $\mathcal{O}_{K} / \mathfrak{N}=$ $\mathbb{Z} / N$. Fix such an $\mathfrak{N}$ and fix a $C M$ elliptic curve $A$ with $\operatorname{End}_{H}(A)=\mathcal{O}_{K}$. Let $H_{\mathfrak{N}} / H$ denote the extension over which the individual $\mathfrak{N}$-torsion points of $A$ are defined, which is an abelian extension of $K$. A choice of $t_{A} \in A[\mathfrak{N}]$ of order $N$ determines a $\Gamma_{1}(N)$-level structure on $A$ defined over any $F / H_{\mathfrak{N}}$. Fix a choice of $t_{A}$ once and for all.

Now consider the set

$$
\operatorname{Isog}(A):=\left\{\left(\varphi, A^{\prime}\right)\right\} / \text { Isomorphism, }
$$

where $A^{\prime}$ is an elliptic curve and $\varphi: A \rightarrow A^{\prime}$ is an isogeny defined over $\bar{K}$, and two pairs $\left(\varphi_{1}, A_{1}^{\prime}\right)$ and $\left(\varphi_{2}, A_{2}^{\prime}\right)$ are isomorphic if there is an isomorphism $\iota: A_{1}^{\prime} \rightarrow A_{2}^{\prime}$ over $\bar{K}$ such that $\iota \varphi_{1}=\varphi_{2}$. Let $\operatorname{Isog}^{\mathfrak{N}}(A) \subset \operatorname{Isog}(A)$ be the subset consisting of isomorphism classes of $\left(\varphi, A^{\prime}\right)$ such that $\operatorname{ker}(\varphi) \cap A[\mathfrak{N}]=\{0\}$. Note that the group $\square^{\mathfrak{N}}$ of $\mathcal{O}_{K}$-ideals relatively prime to $\mathfrak{N}$ act on $\operatorname{Isog}^{\mathfrak{N}}(A)$ via $\mathfrak{a} \star\left(\varphi, A^{\prime}\right)=\left(\varphi_{\mathfrak{a}} \varphi, A^{\prime} / A^{\prime}[\mathfrak{a}]\right)$, where

$$
\varphi_{\mathfrak{a}}: A^{\prime} \rightarrow A^{\prime} / A^{\prime}[\mathfrak{a}]
$$


is the natural surjection. Given triples $\left(A_{1}, t_{1}, \omega_{1}\right)$ and $\left(A_{2}, t_{2}, \omega_{2}\right)$, we define an isogeny from $\left(A_{1}, t_{1}, \omega_{1}\right)$ to $\left(A_{2}, t_{2}, \omega_{2}\right)$ to be an isogeny

$$
\varphi: A_{1} \rightarrow A_{2} \quad \text { such that } \varphi\left(t_{1}\right)=t_{2} \text { and } \varphi^{*}\left(\omega_{2}\right)=\omega_{1} .
$$

We have an action of $\square^{\mathfrak{N}}$ on (isomorphism classes of) triples $\left(A^{\prime}, t^{\prime}, \omega^{\prime}\right)$ with $\operatorname{End}\left(A^{\prime}\right)=\mathcal{O}_{K}$ and $t^{\prime} \in A^{\prime}[\mathfrak{N}]$ given by

$$
\mathfrak{a} \star\left(A^{\prime}, t^{\prime}, \omega^{\prime}\right)=\left(A^{\prime} / A^{\prime}[\mathfrak{a}], \varphi_{\mathfrak{a}}\left(t^{\prime}\right), \omega_{\mathfrak{a}}^{\prime}\right), \quad \text { where } \varphi_{\mathfrak{a}}^{*}\left(\omega_{\mathfrak{a}}^{\prime}\right)=\omega^{\prime} .
$$

Definition 22. Note that a pair $\left(\varphi, A^{\prime}\right) \in \operatorname{Isog}^{\mathfrak{N}}(A)$ determines a point $\left(A^{\prime}, \varphi\left(t_{A}\right)\right) \in$ $Y_{1}(N)\left(H_{\mathfrak{N}}\right) \subset Y_{1}(N)(F)$ and, for any integer $r \geq 0$, an embedding $\left(A^{\prime}\right)^{r} \hookrightarrow W_{r}$. (For the definition of $W_{r}$, see Section 2.2.) Using this we view the graph as being embedded in $X_{r}:=W_{r} \times A^{r}$ :

$$
\Gamma_{\varphi} \subset A^{r} \times\left(A^{\prime}\right)^{r} \subset A^{r} \times W_{r} \cong X_{r} .
$$

We define the generalized Heegner cycle associated with $(\varphi, A)$ as $\Delta_{\varphi}:=\epsilon_{X} \Gamma_{\varphi}$, where $\epsilon_{X}$ is the projector defined in [Bertolini et al. 2013, Section 2.2]. Note that $\Gamma_{\varphi}$ is defined over $H_{\mathfrak{N}}$, and thus determines a class in the Chow group $\mathrm{CH}_{0}^{r+1}\left(X_{r}\right)\left(H_{\mathfrak{N}}\right)$, as defined in Section 2.2.

3.4. Bertolini, Darmon, and Prasanna's p-adic L-function and p-adic Waldspurger formula. Fix a normalized newform $f \in S_{k}\left(\Gamma_{0}(N), \varepsilon_{f}\right)$. Given a Hecke character $\chi$ of infinity type $\left(j_{1}, j_{2}\right)$, recall that the central character $\varepsilon_{\chi}$ of $\chi$ is the finite order character defined by

We define

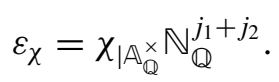

$$
L\left(f, \chi^{-1}, 0\right):=L\left(\pi_{f} \times \pi_{\chi^{-1}}, s-\frac{1}{2}\left(k-1-j_{1}-j_{2}\right)\right),
$$

where $\pi_{f}$ and $\pi_{\chi^{-1}}$ are the (unitarily normalized) automorphic representations associated with $f$ and $\chi$, respectively. The Hecke character $\chi$ is said to be central critical with respect to $f$ if $j_{1}+j_{2}=k$ and either

(1) $1 \leq j_{1}, j_{2} \leq k-1$, or

(2) $j_{1} \geq k$ and $j_{2} \leq 0$,

and if the following condition on the central character of $\chi$ is satisfied:

$$
\varepsilon_{\chi}=\varepsilon_{f}
$$

(Note, since we assume the Heegner hypothesis, this implies that $\chi$ is unramified outside of $\mathfrak{f}\left(\varepsilon_{f}\right)$.) Central criticality of $\chi$ is equivalent to requiring that $\pi_{f} \times \pi_{\chi^{-1}}$ is self-dual and that the point of symmetry for the functional equation of $L\left(f, \chi^{-1}, s\right)$ is $s=0$. 
For central critical $\chi$ with infinity type in range (1) (resp. range (2)) above, the root number satisfies $\epsilon_{\infty}\left(f, \chi^{-1}\right)=-1$ (resp. $\left.\epsilon_{\infty}\left(f, \chi^{-1}\right)=+1\right)$. We henceforth make the auxiliary assumption that

the local root numbers of $L\left(f, \chi^{-1}, s\right)$ satisfy $\epsilon_{\ell}\left(f, \chi^{-1}\right)=+1$ at all finite primes $\ell$.

This assumption is automatic for $\ell \in S_{f}=\left\{\ell: \ell \mid\left(N, D_{K}\right), \ell \nmid \mathfrak{f}\left(\varepsilon_{f}\right)\right\}$ since we assume the Heegner hypothesis, and thus is automatically satisfied when $\left(N, D_{K}\right)=1$, as we assume in Assumptions 1. These conditions on the local root numbers of $L\left(f, \chi^{-1}, s\right)$ imply that the global root number is $\epsilon\left(f, \chi^{-1}\right)=-1$ for $\chi$ in infinity type range (1) above, and $\epsilon\left(f, \chi^{-1}\right)=+1$ for $\chi$ in range (2) above. Thus we have $L\left(f, \chi^{-1}, 0\right)=0$ in range (1) and expect $L\left(f, \chi^{-1}, 0\right) \neq 0$ (generically) in range (2).

For any Hecke character $\varepsilon$ over $\mathbb{Q}$ of conductor $N_{\varepsilon} \mid N$, define $\mathfrak{N}_{\varepsilon}$ to be the unique ideal in $\mathcal{O}_{K}$ that divides $\mathfrak{N}$ and has norm equal to $N_{\varepsilon}$. For a Hecke character $\chi$ over $K$, we say that $\chi$ is of finite type $(\mathfrak{N}, \varepsilon)$ if

$$
\chi_{\mid \hat{\mathcal{O}}_{K}^{\times}}=\psi_{\varepsilon}
$$

where $\psi_{\varepsilon}$ is the composite map

$$
\hat{\mathcal{O}}_{K}^{\times} \rightarrow \prod_{v \mid \mathfrak{N}_{\varepsilon}}\left(\mathcal{O}_{K, v} / \mathfrak{N}_{\varepsilon} \mathcal{O}_{K, v}\right)^{\times} \cong \prod_{\ell \mid N_{\varepsilon}}\left(\mathbb{Z}_{\ell} / N_{\varepsilon} \mathbb{Z}_{\ell}\right)^{\times} \stackrel{\prod_{\ell \mid N_{\varepsilon}} \varepsilon_{\ell}}{\longrightarrow} \mathbb{C}^{\times},
$$

or equivalently,

$$
\hat{\mathcal{O}}_{K}^{\times} \rightarrow\left(\hat{\mathcal{O}}_{K} / \mathfrak{N}_{\varepsilon} \hat{\mathcal{O}}_{K}\right)^{\times} \cong\left(\mathcal{O}_{K} / \mathfrak{N}_{\varepsilon_{K}} \mathcal{O}_{K}\right)^{\times} \cong\left(\mathbb{Z} / N_{\varepsilon} \mathbb{Z}\right)^{\times} \stackrel{\varepsilon^{-1}}{\longrightarrow} \mathbb{C}^{\times} .
$$

Let $\Sigma_{\mathrm{cc}}(\mathfrak{N})$ denote the set of central critical characters of finite type $(\mathfrak{N}, \varepsilon)$ satisfying the above auxiliary condition on local root numbers, with $\mathfrak{f}(\chi) \mid \mathfrak{N}$, and let $\Sigma_{\mathrm{cc}}^{(1)}(\mathfrak{N})$ and $\Sigma_{\mathrm{cc}}^{(2)}(\mathfrak{N})$ denote the subsets of such characters with infinity type $(k+j,-j)$, where $1-k \leq j \leq-1$ and $j \geq 0$, respectively, so that we have $\Sigma_{\mathrm{cc}}(\mathfrak{N})=\Sigma_{\mathrm{cc}}^{(1)}(\mathfrak{N}) \sqcup \Sigma_{\mathrm{cc}}^{(2)}(\mathfrak{N})$.

We now define a field $F^{\prime}$ as follows. Note that $\operatorname{Gal}\left(H_{\mathfrak{N}} / H\right) \cong(\mathbb{Z} / N)^{\times}$, and let $H_{\mathfrak{N}}^{\prime} \subset H_{\mathfrak{N}}$ be the subfield fixed by $\operatorname{ker}\left(\varepsilon_{f}\right)$. The values $\chi(\mathfrak{a})$ generate a finite extension as $\chi$ ranges over $\Sigma_{\mathrm{cc}}^{(2)}(\mathfrak{N})$ and $\mathfrak{a}$ ranges over $\mathbb{A}_{K, f}^{\times}$. Let $F^{\prime}$ be the field which is the compositum of this latter extension with $E_{f}$ and $H_{\mathfrak{N}}^{\prime}$, and let $\mathfrak{p}^{\prime}$ be the prime ideal of $\mathcal{O}_{F^{\prime}}$ above $p$ determined by our embedding $i_{p}$. The set $\Sigma_{\mathrm{cc}}^{(2)}(\mathfrak{N})$ has a natural topology, namely, the topology of uniform convergence induced by the $p$-adic metric on the completion of $F^{\prime}$ in $\overline{\mathbb{Q}}_{p}$, when $\Sigma_{\mathrm{cc}}^{(2)}(\mathfrak{N})$ is viewed as a space of functions on finite idèles prime to $p$. (See [Bertolini et al. 2013, Section 5.2] for details.) Let $\hat{\Sigma}_{\mathrm{cc}}(\mathfrak{N})$ denote the completion of $\Sigma_{\mathrm{cc}}^{(2)}(\mathfrak{N})$ in this topology. As explained in Section 5.3 of loc. cit., we may view $\Sigma_{\mathrm{cc}}^{(1)}(\mathfrak{N})$ as subset of $\hat{\Sigma}_{\mathrm{cc}}(\mathfrak{N})$. 
Definition 23. For a Hecke character over $K$ of infinity type $(a, b)$, we define the signature of $\chi$ as $a-b \in \mathbb{Z}$. For $\chi \in \Sigma_{\mathrm{cc}}^{(2)}(\mathfrak{N})$ of infinity type $(k+j,-j)$, this is $k+2 j$. Given $\chi \in \hat{\Sigma}_{\mathrm{cc}}(\mathfrak{N})$, we choose a Cauchy sequence $\left\{\chi_{i}\right\}_{i=1}^{\infty} \subset \Sigma_{\mathrm{cc}}^{(2)}(\mathfrak{N})$ converging to $\chi$, where $\chi_{i}$ has infinity type $\left(k+j_{i},-j_{i}\right)$. Given $M \in \mathbb{Z}$, there exists an $i(M)$ such that, for all $i_{1}, i_{2} \geq i(M)$, we have $\chi_{i_{1}}(x) \equiv \chi_{i_{2}}(x)\left(\bmod \left(\mathfrak{p}^{\prime}\right)^{M}\right)$ for all $x \in \mathbb{A}_{K, f}^{\times, p}$. Evaluating on $x \in \mathbb{A}_{K, f}^{\times, p}$ congruent to $1(\bmod \mathfrak{N})$, we see that $j_{i_{1}} \equiv j_{i_{2}}\left(\bmod (p-1) p^{M-1}\right)$. Hence, the Cauchy sequence $\left\{j_{i}\right\}_{i=1}^{\infty} \subset \mathbb{Z}$ converges to an element $j \in \mathbb{Z} /(p-1) \times \mathbb{Z}_{p}$. Then the signature of $\chi$ is $k+2 j \in \mathbb{Z} /(p-1) \times \mathbb{Z}_{p}$.

The elliptic curve $A_{0}=\mathbb{C} / \mathcal{O}_{K}$ over $\mathbb{C}$ has complex multiplication by $\mathcal{O}_{K}$, and hence is defined over $H$. A choice of invariant differential $\omega_{0} \in \Omega_{A_{0} / H}^{1}$ determines a complex period $\Omega_{\infty} \in \mathbb{C}^{\times}$via

$$
\omega_{0}=\Omega_{\infty} \cdot 2 \pi i d z
$$

where $z$ is the standard coordinate on $\mathbb{C}$. We now make the assumption that

$p$ is split in $K / \mathbb{Q}$,

so that in particular $i_{p}(K) \subset \mathbb{Q}_{p}$. Let $\mathfrak{p}$ be a prime of $K$ above $p$. Let $\mathcal{A}_{0}$ be a good integral model over $A_{0}$. The completion of $\mathcal{A}_{0}$ along its identity section $\hat{\mathcal{A}}_{0}$ is (noncanonically) isomorphic to $\hat{\mathbb{G}}_{m}$ over $\mathcal{O}_{\mathbb{C}_{p}}$. Fix an isomorphism $\iota: \hat{\mathcal{A}}_{0} \stackrel{\sim}{\longrightarrow} \hat{\mathbb{G}}_{m}$ (which is equivalent to fixing an isomorphism $\mathcal{A}_{0}\left[\mathfrak{p}^{\infty}\right]:=\lim _{\underline{n}} \mathcal{A}_{0}\left[\mathfrak{p}^{n}\right] \stackrel{\sim}{\longrightarrow} \lim \underset{n}{\longrightarrow} \mu_{p^{n}}=: \mu_{p^{\infty}}$, which is determined up to multiplication by a scalar in $\mathbb{Z}_{p}^{\times}$). Let $\omega_{\text {can }}:=\iota^{*} \frac{d u}{u}$. Now we define a $p$-adic period $\Omega_{p} \in \mathbb{C}_{p}^{\times}$via

$$
\omega_{0}=\Omega_{p} \cdot \omega_{\text {can }} .
$$

Bertolini, Darmon, and Prasanna [Bertolini et al. 2013] define an anticyclotomic $p$-adic L-function associated with $f$ and $\chi$, interpolating a twisted trace over CM triples attached to $(f, \chi)$ for $\chi \in \Sigma_{\mathrm{cc}}^{(2)}(\mathfrak{N})$. Recall our fixed triple $\left(A, t_{A}, \omega_{\text {can }}\right)$. Then there is an isogeny $\varphi_{0}: A \rightarrow A_{0}$, and we let $\left(A_{0}, t_{0}, \omega_{0}\right)$ denote the unique triple fitting into an induced isogeny of triples $\varphi_{0}:\left(A, t_{A}, \omega_{\text {can }}\right) \rightarrow\left(A_{0}, t_{0}, \omega_{0}\right)$ in the sense of Section 3.3. Henceforth, fix a complex period $\Omega_{\infty}$ and a $p$-adic period $\Omega_{p}$ associated with $\omega_{0}$. For $\mathfrak{a} \in \square^{\mathfrak{N}}$, let $\varphi_{\mathfrak{a}}: A_{0} \rightarrow \mathfrak{a} \star A_{0}$ be the natural isogeny, so that $\left(\varphi_{\mathfrak{a}} \varphi_{0}, A\right) \in \operatorname{Isog}^{\mathfrak{N}}(A)$.

Theorem 24 [Bertolini et al. 2013, Theorem 5.9]. Fix a normalized newform $f$ in $S_{k}\left(\Gamma_{0}(N), \varepsilon_{f}\right)$. Suppose $p$ is a prime split in $K / \mathbb{Q}$. Let $\{\mathfrak{a}\}$ be a set of representatives of $\mathrm{Cl}\left(\mathcal{O}_{K}\right)$ which are prime to $\mathfrak{N}$. Then there exists a unique continuous function $\hat{\Sigma}_{\mathrm{cc}}(\mathfrak{N}) \rightarrow \mathbb{C}_{p}: \chi \mapsto \mathcal{L}_{p}(f, \chi)$ satisfying

$$
\mathcal{L}_{p}(f, \chi)=\left(\sum_{[\mathfrak{a}] \in \operatorname{Cl}\left(\mathcal{O}_{K}\right)}\left(\chi_{j}\right)^{-1}(\mathfrak{a}) \cdot\left(\theta^{j} f\right)^{b}\left(\mathfrak{a} \star\left(A_{0}, t_{0}, \omega_{\text {can }}\right)\right)\right)^{2}
$$


for all $\chi \in \Sigma_{\mathrm{cc}}^{(2)}(\mathfrak{N})$ of infinity type $(k+j,-j)$ with $j \geq 0$.

Bertolini, Darmon, and Prasanna's $p$-adic Waldspurger formula, which relates the special value of $\mathcal{L}_{p}(f, \chi)$ at some $\chi \in \Sigma_{\mathrm{cc}}^{(1)}(\mathfrak{N}) \subset \hat{\Sigma}_{\mathrm{cc}}(\mathfrak{N})$ to the $p$-adic AbelJacobi image of a certain generalized Heegner cycle, is the main result of [Bertolini et al. 2013].

Theorem 25 [Bertolini et al. 2013, Theorem 5.13]. Suppose $\chi \in \Sigma_{\mathrm{cc}}^{(1)}(\mathfrak{N})$ has infinity type $(k-1-j, 1+j)$, where $0 \leq j \leq r=k-2$. Then

$$
\begin{aligned}
\frac{\mathcal{L}_{p}(f, \chi)}{\Omega_{p}^{2(r-2 j)}=(1-} & \left.\chi^{-1}(\overline{\mathfrak{p}}) a_{p}(f)+\chi^{-2}(\overline{\mathfrak{p}}) \varepsilon_{f}(p) p^{k-1}\right)^{2} \\
& \times\left(\frac{1}{\Gamma(j+1)} \sum_{[\mathfrak{a}] \in \operatorname{Cl}\left(\mathcal{O}_{K}\right)}\left(\chi \mathbb{N}_{K}\right)^{-1}(\mathfrak{a}) \cdot \mathrm{AJ}_{F_{\mathfrak{p}^{\prime}}}\left(\Delta_{\varphi_{\mathfrak{a}} \varphi_{0}}\right)\left(\omega_{f} \wedge \omega_{A}^{j} \eta_{A}^{r-j}\right)\right)^{2} .
\end{aligned}
$$

Here $\mathrm{AJ}_{F_{\mathfrak{p}^{\prime}}^{\prime}}$ is the p-adic Abel-Jacobi map defined in [Bertolini et al. 2013, Section $3.4]$, and $\Delta_{\varphi_{\mathfrak{a}} \varphi_{0}}$ is the generalized Heegner cycle attached to $\left(\varphi_{\mathfrak{a}} \varphi_{0}, A\right) \in \operatorname{Isog}^{\mathfrak{N}}(A)$ as defined in Definition 22.

Suppose $\chi$ is a finite order Hecke character on $K$. We define the Heegner point attached to $\chi$ as

$$
P(\chi):=\sum_{\sigma \in \operatorname{Gal}\left(H_{\mathfrak{N}} / K\right)} \chi^{-1}(\sigma) \cdot \Delta^{\sigma} \in J_{1}(N)\left(H_{\mathfrak{N}}\right) \otimes_{\mathcal{O}_{E_{f}}} E_{f, \chi}
$$

where $\Delta$ equals $\left[\left(A_{0}, t, \omega_{0}\right)\right]-(\infty) \in J_{1}(N)\left(H_{\mathfrak{N}}\right)$ and the isomorphism class $\left[\left(A_{0}, t, \omega_{0}\right)\right]$ is viewed as a point in $X_{1}(N)\left(H_{\mathfrak{N}}\right)$. The embedding $i_{p}: \overline{\mathbb{Q}} \hookrightarrow \mathbb{C}_{p}$ allows us to define the formal group logarithm on the formal group by

$$
\log _{\omega_{f}}: \hat{J}_{1}(N)\left(\mathfrak{p}^{\prime} \mathcal{O}_{F_{\mathfrak{p}^{\prime}}^{\prime}}\right) \rightarrow \mathbb{C}_{p},
$$

which we extend to a map

$$
\log _{\omega_{f}}: J_{1}(N)\left(F_{\mathfrak{p}^{\prime}}^{\prime}\right) \otimes_{\mathcal{O}_{E_{f}}} E_{f, \chi} \rightarrow \mathbb{C}_{p}
$$

by linearity.

For $k=2$, we have $\mathrm{AJ}_{F_{\mathfrak{p}^{\prime}}^{\prime}}(P(\chi))\left(\omega_{f}\right)=\log _{\omega_{f}} P(\chi)$. Thus Theorem 25 becomes:

Theorem 26. Suppose $\chi$ is a finite order Hecke character on $K$ with $\chi \mathbb{N}_{K} \in \Sigma_{\mathrm{cc}}^{(1)}(\mathfrak{N})$. Then

$$
\mathcal{L}_{p}\left(f, \chi \mathbb{N}_{K}\right)=\left(1-\chi^{-1}(\overline{\mathfrak{p}}) p^{-1} a_{p}(f)+\chi^{-2}(\overline{\mathfrak{p}}) \varepsilon_{f}(p) p^{-1}\right)^{2} \log _{\omega_{f}}^{2} P(\chi) .
$$

3.5. Katz's p-adic L-function and Gross's factorization on the cyclotomic line. We can view any $p$-adic Hecke character $\chi: K^{\times} \backslash \mathbb{A}_{K}^{\times} \rightarrow \mathbb{C}_{p}^{\times}$as a Galois character $\chi: \operatorname{Gal}(\bar{K} / K) \rightarrow \mathcal{O}_{\mathbb{C}_{p}}^{\times}$via the Artin isomorphism of class field theory. Let $\mathfrak{C}$ be an integral ideal of $\mathcal{O}_{K}$ which is prime to $p$. Let $K\left(\mathfrak{C} p^{r}\right)$ denote the ray class field 
of $K$ of conductor $\mathfrak{C} p^{r}$ and let $K\left(\mathfrak{C} p^{\infty}\right)=\bigcup_{r} K\left(\mathfrak{C} p^{r}\right)$. Given a $p$-adic character $\chi$ arising from a type $A_{0}$ algebraic Hecke character, denote its complex counterpart by $(\chi)_{\infty}$. (Throughout this section only, we will make this distinction between complex and $p$-adic avatars.)

We recall Katz's $p$-adic $L$-function for CM fields (applied to our fixed imaginary quadratic field $K$ ), as well as Hida and Tilouine's extension [1993] to the case of general auxiliary conductor. Here we use the normalization found in [Gross 1980] (with $\delta=\sqrt{D_{K}} / 2$ and so $\mathfrak{c}=1$, in the notation of [Katz 1978, Theorem 5.3.0]). Fix a decomposition $\mathfrak{C}=\mathfrak{F} \mathfrak{F}_{c} \mathfrak{I}$, where $\mathfrak{F F}_{c}$ consists of split primes in $K, \overline{\mathfrak{F}}$ is a subset of $\mathfrak{F}_{c}$, and $\mathfrak{I}$ consists of inert or ramified primes in $K$. Recall our fixed identification $i: \mathbb{C} \stackrel{\sim}{\longrightarrow} \mathbb{C}_{p}$, the prime $\mathfrak{p} \mid p$ determining $K \hookrightarrow \overline{\mathbb{Q}}_{p}$, and the periods $\Omega_{p}$ and $\Omega_{\infty}$ defined in Section 3.4.

Theorem 27 ([Katz 1978]; see also [Hida and Tilouine 1993]). There exists a unique p-adic analytic function $\chi \mapsto L_{p}(\chi, 0)$ for $\chi: \operatorname{Gal}\left(K\left(\mathfrak{C} p^{\infty}\right) / K\right) \rightarrow \mathbb{C}_{p}^{\times}$ which satisfies (under our fixed identification $i: \mathbb{C} \stackrel{\sim}{\longrightarrow} \mathbb{C}_{p}$ )

$$
\begin{aligned}
L_{p}(\chi, 0)=4 \operatorname{Local}_{\mathfrak{p}}(\chi)(-1)^{k_{1}+k_{2}}\left(\frac{\Omega_{p}}{\Omega_{\infty}}\right)^{k_{1}-k_{2}}\left(\frac{2 \pi i}{\sqrt{D_{K}}}\right)^{-k_{2}} \\
\quad \times\left(k_{1}-1\right) !(1-\chi(\overline{\mathfrak{p}}))(1-\check{\chi}(\overline{\mathfrak{p}})) L(\chi, 0) \prod_{v \mid \mathfrak{C}}(1-\chi(v))
\end{aligned}
$$

for characters $\chi$ with infinity type $\left(-k_{1},-k_{2}\right)$, where $k_{1} \geq 1$ and $k_{2} \leq 0$, and such that $\mathfrak{f}(\chi)$ is divisible by all the prime factors of $\mathfrak{F}$. Here $\operatorname{Local}_{\mathfrak{p}}(\chi)$ is a complex scalar associated with our fixed embedding $K \hookrightarrow \overline{\mathbb{Q}}_{p}$, as in [Katz 1978]. (We have in fact that $\operatorname{Local}_{\mathfrak{p}}(\chi)=1$ if $\chi$ is unramified at $\mathfrak{p}$.)

Recall the dual character $\check{\chi}$ defined by $\check{\chi}(\mathfrak{a})=\chi^{-1}(\overline{\mathfrak{a}}) \mathbb{N}_{K}(\mathfrak{a})$. The function $L_{p}(\chi, 0)$ satisfies the functional equation

$$
W\left((\chi)_{\infty}, \sqrt{D_{K}}\right) L_{p}(\check{\chi}, 0)=L_{p}(\chi, 0),
$$

where $W\left((\chi)_{\infty}, \delta\right)$ is given by

$$
\begin{gathered}
W\left((\chi)_{\infty}, \delta\right)=\prod_{v \mid \mathfrak{F}} G\left((\chi)_{\infty, v}^{-1}, \bar{\delta}\right) \prod_{v \mid \mathfrak{F}_{c}} G\left((\chi)_{\infty, \bar{v}}^{-1}, \delta\right) \prod_{v \mid \mathfrak{I}} G\left((\chi)_{\infty, v}^{-1}, \delta\right), \\
G\left((\chi)_{\infty, v}, \delta\right)=(\chi)_{\infty, v}\left(\pi_{v}^{-e}\right) \sum_{u \in\left(\mathcal{O}_{K, v} / \pi_{v}^{e} \mathcal{O}_{K, v}\right)^{\times}}(\chi)_{\infty, v}(u) \Psi_{K, v}\left(-u \pi_{v}^{-e} \delta^{-1}\right),
\end{gathered}
$$

$e$ is the exponent of $v$ in $\mathfrak{f}(\chi), \pi_{v}$ is a local uniformizer of $K_{v}$, and $\Psi_{K}: \mathbb{A}_{K} \rightarrow \mathbb{C}$ is the standard additive character normalized so that $\Psi_{K, \infty}\left(x_{\infty}\right)=\exp \left(2 \pi i \operatorname{Tr}_{\mathbb{C} / \mathbb{R}}\left(x_{\infty}\right)\right)$.

Henceforth, set

$$
G\left((\chi)_{\infty}, \delta\right)=\prod_{v \mid \mathfrak{F}} G\left((\chi)_{\infty, v}, \delta\right)
$$


Now define the $p$-adic $L$-function

$$
L_{p}(\chi, s):=L_{p}\left(\chi\left\langle\mathbb{N}_{K}\right\rangle^{s}, 0\right)
$$

Gross gives the following factorization of $L_{p}$ along the cyclotomic line.

Theorem 28 [Gross 1980]. In the situation of Theorem 27, suppose that $\mathfrak{F}_{c}=\overline{\mathfrak{F}}$, $\mathfrak{I}=1$ and $\mathfrak{C}=f \mathcal{O}_{K}=\mathfrak{F} \overline{\mathfrak{F}}$, where $f \in \mathbb{Z}_{>0}$. Suppose $\chi: \operatorname{Gal}\left(K\left(\mu_{f p^{\infty}}\right) / \mathbb{Q}\right) \rightarrow \mathbb{C}_{p}^{\times}$is a continuous p-adic Galois character which is trivial on complex conjugation (and so corresponds to an even $p$-adic Hecke character), and let $\chi_{/ K}$ be the restriction of $\chi$ to $\operatorname{Gal}\left(K\left(\mu_{f p^{\infty}}\right) / K\right)$. Then

$$
\frac{\langle f\rangle^{s}}{\prod_{\ell \mid f} \chi_{\ell}^{-1}\left(-\sqrt{D_{K}}\right) \mathfrak{g}_{\ell}(\chi)} L_{p}(\chi / K, s)=L_{p}\left(\chi \varepsilon_{K} \omega, s\right) L_{p}\left(\chi^{-1}, 1-s\right)
$$

for $s \in \mathbb{Z}_{p}$, where $f \in \mathbb{Z}_{>0}$ is viewed as $f=\omega(f)\langle f\rangle \in \mu_{2(p-1)} \times\left(1+2 p \mathbb{Z}_{p}\right)=\mathbb{Z}_{p}^{\times}$, and on the right are Kubota-Leopoldt p-adic L-functions for p-adic Hecke characters over $\mathbb{Q}$.

Remark 29. Gross [1980] originally proves Theorem 28 for auxiliary conductor $f=1$, even though his proof translates mutatis mutandis to the case of arbitrary $f$. The general auxiliary conductor version of the theorem seems to be widely present in the literature, although the author has not been able to locate a complete proof. Thus, for the sake of completeness, we give a proof of Theorem 28, following Gross's method. We will first need a lemma.

Lemma 30. In the situation of Theorem 27, suppose that $\mathfrak{F}_{c}=\overline{\mathfrak{F}}, \mathfrak{I}=1$ and $\mathfrak{C}=f \mathcal{O}_{K}=\mathfrak{F} \overline{\mathfrak{F}}$. Suppose $\chi=\tilde{\chi} \circ \mathbb{N}_{K}$ for some Hecke character $\tilde{\chi}$ over $\mathbb{Q}$. Then

$$
W\left((\chi)_{\infty}, \delta\right)=\frac{G\left((\chi)_{\infty}^{-1}, \bar{\delta}\right)}{G\left((\check{\chi})_{\infty}^{-1},-\bar{\delta}\right)}
$$

Proof. Since $\chi=\tilde{\chi} \circ \mathbb{N}_{K}$, we have $\chi_{v}(x)=\chi_{\bar{v}}(\bar{x})$ for any place $v$ of $K$. For each $v \mid \mathfrak{F} \overline{\mathfrak{F}}$, since $K_{v}=\mathbb{Q}_{\ell}$ (where $\ell$ is the rational prime below $v$ ), we can choose representatives $\{u\}$ of $\left(\mathcal{O}_{K} / \pi_{v}^{e} \mathcal{O}_{K}\right)^{\times}=\left(\mathbb{Z} / \ell^{e} \mathbb{Z}\right)^{\times}$such that $\bar{u}=u$ and local uniformizers $\pi_{v}$ such that $\overline{\pi_{v}}=\pi_{v}$. (This is accomplished by replacing $\pi_{v}$ with $\pi_{v} \overline{\pi_{v}}$ if needed.) 
Thus, we have, for any $v \mid \mathfrak{F}$,

$$
\begin{aligned}
& G\left((\check{\chi})_{\infty, v}^{-1}, \delta\right) \\
& \quad=(\check{\chi})_{\infty, v}^{-1}\left(\pi_{v}^{-e}\right) \sum_{u \in\left(\mathcal{O}_{K, v} / \pi_{v}^{e} \mathcal{O}_{K, v}\right)^{\times}}(\check{y})^{-1}(u) \Psi_{K, v}\left(-u \pi_{v}^{-e} \delta^{-1}\right) \\
& \left.\quad=\left(\mathbb{N}_{K}\right)_{\infty, v}^{-1}\left(\pi_{v}^{-e}\right)(\chi)_{\infty, \bar{v}}\left(\overline{\pi_{v}^{-e}}\right) \sum_{u \in\left(\mathcal{O}_{K, v} / \pi_{v}^{e} \mathcal{O}_{K, v}\right)^{\times}}\left(\mathbb{N}_{\infty}\right)_{\infty, v}^{-1}(u)(\chi)_{\infty, \bar{v}}(\bar{u}) \overline{\Psi_{K, v}\left(\overline{u \pi_{v}^{-e} \delta^{-1}}\right.}\right) \\
& \left.\quad=\left(\mathbb{N}_{K}\right)_{\infty, v}^{-1}\left(\pi_{v}^{-e}\right)(\chi)_{\infty, v}\left(\pi_{v}^{-e}\right) \sum_{u \in\left(\mathcal{O}_{K, v} / \pi_{v}^{e} \mathcal{O}_{K, v}\right)^{\times}} \overline{\sum_{\infty, v}(u) \Psi_{K, v}\left(u \pi_{v}^{-e} \overline{\delta^{-1}}\right.}\right) \\
& \quad=(\chi)_{\infty, v}\left(\pi_{v}^{-e}\right)\left(\sum_{u \in\left(\mathcal{O}_{K, v} / \pi_{v}^{e} \mathcal{O}_{K, v}\right)^{\times}}(\chi)_{\infty, v}^{-1}(u) \Psi_{K, v}\left(u \pi_{v}^{-e} \overline{\delta^{-1}}\right)\right)^{-1} \\
& =\left(G\left((\chi)_{\infty, v}^{-1},-\bar{\delta}\right)\right)^{-1},
\end{aligned}
$$

where in the penultimate equality, we have used the fact that

$$
\left|\sum_{u \in\left(\mathcal{O}_{K, v} / \pi_{v}^{e} \mathcal{O}_{K, v}\right)^{\times}}(\chi)_{\infty, v}^{-1}(u) \Psi_{K, v}\left(-u \pi_{v}^{-e} \overline{\delta^{-1}}\right)\right|^{2}=\left(\mathbb{N}_{K}\right)_{\infty, v}\left(\pi_{v}^{-e}\right) .
$$

Now simply note that, by definition of $W\left((\chi)_{\infty}, \delta\right)$ and the above computation,

$$
W\left((\chi)_{\infty}, \delta\right)=\prod_{v \mid \mathfrak{F}} G\left((\chi)_{\infty, v}^{-1}, \bar{\delta}\right) \prod_{v \mid \mathfrak{F}} G\left((\chi)_{\infty, v}^{-1}, \delta\right)=\frac{G\left((\chi)_{\infty}^{-1}, \bar{\delta}\right)}{G\left((\check{\chi})_{\infty}^{-1},-\bar{\delta}\right)}
$$

Proof of Theorem 28. Interpreting the statement as an assertion about $p$-adic measures, and proceeding as in [Gross 1980] using Lemma 1.1 of loc. cit., it suffices to prove the theorem at $s=0$ and when $\chi$ is of finite order.

Let $\chi$ be unramified at each place dividing $D_{K}$. Then $\chi: \operatorname{Gal}\left(K\left(\mu_{f p^{r}}\right) / \mathbb{Q}\right) \rightarrow \mathbb{C}_{p}^{\times}$ is an even Dirichlet character, where $f p^{r} \mathcal{O}_{K}=\mathfrak{f}\left(\chi_{/ K}\right),(f, p)=1$ and $f p^{r}>1$. Let $w_{f p^{r}}$ denote the number of roots of unity in $K^{\times}$congruent to $1\left(\bmod f p^{r}\right)$, and let $\mathrm{Cl}\left(f p^{r}\right)$ denote the ray class group of $\mathcal{O}_{K}$ of modulus $f p^{r} \mathcal{O}_{K}$. Recall our fixed embeddings $i_{\infty}: \overline{\mathbb{Q}} \hookrightarrow \mathbb{C}$ and $i_{p}: \overline{\mathbb{Q}} \hookrightarrow \mathbb{C}_{p}$ compatible with the identification $i: \mathbb{C} \stackrel{\sim}{\longrightarrow} \mathbb{C}_{p}$. For $a \in\left(\mathbb{Z} / f p^{r}\right)^{\times}$, let

$$
C_{f p^{r}}(a)_{\infty}=1-e^{\frac{-2 \pi i a}{f p^{r}}}, \quad C_{f p^{r}}(a)=i_{\infty}^{-1}\left(C_{f p^{r}}(a)_{\infty}\right), \quad C_{f p^{r}}(a)_{p}=i^{-1}\left(C_{f p^{r}}(a)_{\infty}\right) .
$$

Further, let $E_{f p^{r}}(c)_{\infty} \in \overline{\mathbb{Q}}^{\times} \subset \mathbb{C}^{\times}$be the "elliptic units" of [Robert 1973], and let

$$
E_{f p^{r}}(c)=i_{\infty}^{-1}\left(E_{f p^{r}}(c)_{\infty}\right), \quad E_{f p^{r}}(c)_{p}=i\left(E_{f p^{r}}(c)_{\infty}\right) .
$$


Now, for $a \in\left(\mathbb{Z} / f p^{r}\right)^{\times}$, choose $c \in \mathrm{Cl}\left(f p^{r}\right)$ so that $\mathrm{Nm}_{K / \mathbb{Q}}(c) \equiv a\left(\bmod f p^{r}\right)$, and let

$$
F_{f p^{r}}(a)=\mathrm{Nm}_{K\left(f p^{r}\right) / K\left(\mu_{f p^{r}}\right)}\left(E_{f p^{r}}(c)\right) .
$$

Finally, for $a \in A=\left(\mathbb{Z} / f p^{r}\right)^{\times} /\{ \pm 1\}$, let

$$
F^{+}(a)=F_{f p^{r}}(a) F_{f p^{r}}(-a), \quad C^{+}(a)=C_{f p^{r}}(a) C_{f p^{r}}(-a) .
$$

Note that in our situation, for any $s \in \mathbb{Z}_{p}$,

$$
\begin{aligned}
G\left(\left(\chi_{/ K}\left\langle\mathbb{N}_{K}\right\rangle^{s}\right)_{\infty}^{-1},-\sqrt{D_{K}}\right) & =\prod_{v \mid \mathfrak{F}} G\left(\left(\chi_{/ K}\left\langle\mathbb{N}_{K}\right\rangle^{s}\right)_{\infty, v}^{-1},-\sqrt{D_{K}}\right) \\
& =\langle f\rangle^{-s} \prod_{\ell \mid f} \mathfrak{g}_{\ell}(\chi) \chi_{\ell}^{-1}\left(\sqrt{D_{K}}\right)
\end{aligned}
$$

where $f$ is viewed as in $\mathbb{Z}_{p}^{\times}$. Hence, using the special value formulas from [Katz 1976, Formulas 10.4.9-10.4.12] (suitably modified with respect to the normalization of the $p$-adic $L$-function in [Gross 1980]) we have

$\frac{1}{\prod_{\ell \mid f} \chi_{\ell}^{-1}\left(-\sqrt{D_{K}}\right) \mathfrak{g}_{\ell}(\chi)} L_{p}\left(\chi_{/ K}, 0\right)$

$$
\begin{aligned}
& =\frac{\prod_{\ell \mid f} \chi_{\ell}(-1)}{G\left(\left(\chi_{/ K}\right)_{\infty}^{-1},-\sqrt{D_{K}}\right)} L_{p}\left(\chi_{/ K}, 0\right) \\
& =-\frac{1}{3 f p^{r} w_{f p^{r}}}\left(1-\chi_{/ K}(\overline{\mathfrak{p}})\right)\left(1-\frac{\chi_{/ K}^{-1}(\mathfrak{p})}{p}\right) \frac{\mathfrak{g}\left(\chi^{-1}\right)}{f p^{r}} \sum_{a \in A} \chi(a) \log _{p} F^{+}(a)_{p},
\end{aligned}
$$

and

$$
\begin{aligned}
L_{p}\left(\chi \varepsilon_{K} \omega, 0\right) & =-\left(1-\chi \varepsilon_{K}(p)\right) B_{1, \chi \varepsilon_{K}}, \\
L_{p}\left(\chi^{-1}, 1\right) & =-\left(1-\frac{\chi^{-1}(p)}{p}\right) \frac{\mathfrak{g}\left(\chi^{-1}\right)}{f p^{r}} \sum_{a \in A} \chi(a) \log _{p} C^{+}(a)_{p} .
\end{aligned}
$$

On the complex side, we have (by Kronecker's second limit formula; see [Stark 1977])

$$
\begin{aligned}
L^{\prime}\left((\chi / K)_{\infty}, 0\right) & =-\frac{1}{6 f p^{r} w_{f p^{r}}} \sum_{a \in A}(\chi)_{\infty}(a) \log F^{+}(a)_{\infty}, \\
L\left((\chi)_{\infty} \varepsilon_{K}, 0\right) & =-B_{1,(\chi)_{\infty} \varepsilon_{K}} \\
L\left((\chi)_{\infty}^{-1}, 1\right) & =-\frac{\mathfrak{g}\left((\chi)_{\infty}^{-1}\right)}{f p^{r}} \sum_{a \in A}(\chi)_{\infty}(a) \log C^{+}(a)_{\infty},
\end{aligned}
$$


and thus, by the functional equation, we have

$$
L^{\prime}\left((\chi)_{\infty}, 0\right)=-\frac{1}{2} \sum_{a \in A}(\chi)_{\infty}(a) \log C^{+}(a)_{\infty},
$$

where $\log : \mathbb{R}^{\times} \rightarrow \mathbb{R}$ is the map $x \mapsto \log |x|$. Now we claim that

$$
\frac{1}{\prod_{\ell \mid f} \chi_{\ell}^{-1}\left(-\sqrt{D_{K}}\right) \mathfrak{g}_{\ell}(\chi)} L_{p}(\chi / K, 0)=L_{p}\left(\chi \varepsilon_{K} \omega, 0\right) L_{p}\left(\chi^{-1}, 1\right) .
$$

Note that

$$
\left(1-\chi_{/ K}(\overline{\mathfrak{p}})\right)\left(1-\frac{\chi_{/ K}^{-1}(\mathfrak{p})}{p}\right)=\left(1-\chi \varepsilon_{K}(p)\right)\left(1-\frac{\chi^{-1}(p)}{p}\right)
$$

since $\varepsilon_{K}(p)=1$. If this quantity is 0 then the above identity of special values of $p$-adic $L$-functions is trivial, so assume this is not the case. By the above formulas, this identity is equivalent to

$$
-3 f p^{r} w_{f p^{r}} B_{1, \chi \varepsilon_{K}} \sum_{a \in A} \chi(a) \log _{p} C^{+}(a)_{p}=\sum_{a \in A} \chi(a) \log _{p} F^{+}(a)_{p} .
$$

From the complex factorization

$$
L\left(\left(\chi_{/ K}\right)_{\infty}, s\right)=L\left((\chi)_{\infty} \varepsilon_{K}, s\right) L\left((\chi)_{\infty}, s\right),
$$

we get, by taking the derivative at $s=0$,

$$
L^{\prime}\left(\left(\chi_{/ K}\right)_{\infty}, 0\right)=L\left((\chi)_{\infty} \varepsilon_{K}, s\right) L^{\prime}\left((\chi)_{\infty}, 0\right),
$$

which is equivalent, by the above formulas, to

$$
-3 f p^{r} w_{f p^{r}} B_{1,(\chi)_{\infty} \varepsilon_{K}} \sum_{a \in A}(\chi)_{\infty}(a) \log C^{+}(a)_{\infty}=\sum_{a \in A}(\chi)_{\infty}(a) \log F^{+}(a)_{\infty} .
$$

Now $C^{+}(a)_{\infty}$ and $F^{+}(a)_{\infty}$ are $p$-units in the field $M_{\infty}=\mathbb{Q}\left(\cos \frac{2 \pi i}{f p^{r}}\right)$. Let $E\left(M_{\infty}\right)$ denote the group of all $p$-units viewed as a finitely generated subgroup of $\mathbb{R}^{\times}$. Then we claim that the identity

$$
-3 f p^{r} w_{f p^{r}} B_{1,(\chi)_{\infty} \varepsilon_{K}} \sum_{a \in A}(\chi)_{\infty}(a) \otimes C^{+}(a)_{\infty}=\sum_{a \in A}(\chi)_{\infty}(a) \otimes F^{+}(a)_{\infty}
$$

holds in $\mathbb{C} \otimes_{\mathbb{Z}} E\left(M_{\infty}\right)$. The representation of $A=\operatorname{Gal}\left(M_{\infty} / \mathbb{Q}\right)$ on this complex vector space is isomorphic to the regular representation, and the elements

$$
\sum_{a \in A}(\chi)_{\infty}(a) C^{+}(a)_{\infty}, \quad \sum_{a \in A}(\chi)_{\infty}(a) F^{+}(a)_{\infty}
$$


are both in the $(\chi)_{\infty}^{-1}$-eigenspace. Because this eigenspace is one-dimensional, the elements above differ by a complex scalar. Applying the $\mathbb{C}$-linear map

$$
\mathbb{C} \otimes_{\mathbb{Z}} E\left(M_{\infty}\right) \stackrel{1 \otimes \log }{\longrightarrow} \mathbb{R} \otimes \mathbb{C} \stackrel{\text { mult }}{\longrightarrow} \mathbb{C}
$$

and considering the identity above concerning special values of complex $L$-functions, we identify this scalar as $-3 f p^{r} w_{f p^{r}} B_{1,(\chi)_{\infty} \varepsilon_{K}}$. Thus our identity in $\mathbb{C} \otimes_{\mathbb{Z}} E\left(M_{\infty}\right)$ holds. Now applying our identification $i: \mathbb{C} \stackrel{\sim}{\longrightarrow} \mathbb{C}_{p}$, we obtain the identity

$$
-3 f p^{r} w_{f p^{r}} B_{1, \chi \varepsilon_{K}} \sum_{a \in A} \chi(a) \otimes C^{+}(a)_{\infty}=\sum_{a \in A} \chi(a) \otimes F^{+}(a)_{\infty}
$$

in $\mathbb{C}_{p} \otimes E\left(M_{p}\right)$, where $M_{p}=i\left(M_{\infty}\right)$. Finally, applying the homomorphism

$$
\mathbb{C}_{p} \otimes \mathbb{C}_{p} \stackrel{1 \otimes \log _{p}}{\longrightarrow} \mathbb{C}_{p} \otimes \mathbb{C}_{p} \stackrel{\text { mult }}{\longrightarrow} \mathbb{C}_{p},
$$

we obtain our identity of special values of $p$-adic $L$-functions. Now, to extend to general $\chi$ (including when $\chi$ is ramified at places dividing $D_{K}$ ), we use the functional equation of Theorem 27 and Lemma 30:

$$
\begin{aligned}
& \frac{\langle f\rangle^{s}}{\prod_{\ell \mid f} \chi_{\ell}^{-1}\left(-\sqrt{D_{K}}\right) \mathfrak{g}_{\ell}(\chi)} L_{p}\left(\chi_{/ K}, s\right) \\
& =\frac{1}{G\left(\left(\chi_{/ K}\left\langle\mathbb{N}_{K}\right\rangle^{s}\right)_{\infty}^{-1},-\sqrt{D_{K}}\right)} L_{p}\left(\chi_{/ K}, s\right) \\
& =\frac{W\left(\left(\chi_{/ K}\left\langle\mathbb{N}_{K}\right\rangle^{s}\right)_{\infty}, \sqrt{D_{K}}\right)}{G\left(\left(\chi_{/ K}\left\langle\mathbb{N}_{K}\right\rangle^{s}\right)_{\infty}^{-1},-\sqrt{D_{K}}\right)} L_{p}\left(\chi_{/ K}^{-1} \omega_{K}^{-1}, 1-s\right) \\
& =\frac{1}{G\left(\left(\check{\chi}_{/ K}\left\langle\mathbb{N}_{K}\right\rangle^{-s}\right)_{\infty}^{-1}, \sqrt{D_{K}}\right)} L_{p}\left(\left(\chi \omega \varepsilon_{K}\right)_{/ K}^{-1}, 1-s\right) \\
& =\frac{\langle f\rangle^{1-s}}{\prod_{\ell \mid f}\left(\chi \omega \varepsilon_{K}\right)_{\ell}\left(-\sqrt{D_{K}}\right) \mathfrak{g}_{\ell}\left(\left(\chi \omega \varepsilon_{K}\right)^{-1}\right)} L_{p}\left(\left(\chi \omega \varepsilon_{K}\right)_{/ K}^{-1}, 1-s\right) .
\end{aligned}
$$

Here $\check{\chi}_{/ K}$ denotes the dual of $\chi_{/ K}$ and we have used the fact that, for $\ell \mid f$, the characters $\omega$ and $\varepsilon_{K}$ are unramified at $\ell$, as well as the equalities $\varepsilon_{K, \ell}(\ell)=1$, since $\ell$ is split in $K$, and $\left(\varepsilon_{K}\right)_{/ K}=1$.

3.6. Eisenstein descent. We now define the notion of cuspforms which have Eisenstein descent. In this setting, we prove a congruence between the BDP $p$-adic $L$-function and the Katz $p$-adic $L$-function on the anticyclotomic line.

Definition 31. Fix a global or local field $M$ containing $E_{f}$ and fix an integral ideal $\mathrm{m}$ of $\mathcal{O}_{M}$. Suppose $\left(N_{+}, N_{-}, N_{0}\right)$ is a triple of pairwise coprime positive integers, where $N=N_{+} N_{-} N_{0}, N_{+} N_{-}$is squarefree, $N_{0}$ is squarefull, and $\psi_{1}$ and $\psi_{2}$ are Dirichlet characters over $\mathbb{Q}$. We say that a normalized newform $f=\sum_{n=1}^{\infty} a_{n} q^{n} \in$ 
$S_{k}\left(\Gamma_{0}(N), \varepsilon_{f}\right)$, where $k$ is a positive integer, has partial Eisenstein descent of type $\left(\psi_{1}, \psi_{2}, N_{+}, N_{-}, N_{0}\right)($ over $M) \bmod \mathfrak{m}$ if $\psi_{1} \psi_{2}=\varepsilon_{f}$ and if we have

(1) $a_{\ell} \equiv \psi_{1}(\ell)+\psi_{2}(\ell) \ell^{k-1}(\bmod \mathfrak{m})$ for $\ell \nmid N$,

(2) $a_{\ell} \equiv \psi_{1}(\ell)(\bmod \mathfrak{m})$ for $\ell \mid N_{+}$,

(3) $a_{\ell} \equiv \psi_{2}(\ell) \ell^{k-1}(\bmod \mathfrak{m})$ for $\ell \mid N_{-}$,

(4) $a_{\ell} \equiv 0(\bmod \mathfrak{m})$ for $\ell \mid N_{0}$.

If, further, $f$ satisfies

$$
\begin{aligned}
\delta_{\psi_{1}=1} \frac{B_{1, \psi_{2}} B_{k, \psi_{1}}}{k} \prod_{\ell \mid N_{+}}\left(1-\psi_{1}(\ell) \ell^{k-1}\right) \prod_{\ell \mid N_{-}}\left(1-\psi_{2}(\ell)\right) \\
\quad \times \prod_{\ell \mid N_{0}}\left(1-\psi_{1}(\ell) \ell^{k-1}\right)\left(1-\psi_{2}(\ell)\right) \equiv 0(\bmod \mathfrak{m}),
\end{aligned}
$$

where

$$
\delta_{\psi=1}:= \begin{cases}1 & \text { if } \psi=1 \\ 0 & \text { otherwise }\end{cases}
$$

then we say $f$ has (full) Eisenstein descent of type $\left(\psi_{1}, \psi_{2}, N_{+}, N_{-}, N_{0}\right) \bmod \mathfrak{m}$.

Remark 32. Recall, for $k \geq 2$, that the Eisenstein series $E_{k}^{\psi_{1}, \psi_{2},(N)}$ is an element of $N_{k}\left(\Gamma_{0}(N), \psi_{1} \psi_{2}\right)$. (When either $k>2$ or $\psi_{1} \neq 1$ or $\psi_{2} \neq 1$, then in fact $E_{k}^{\psi_{1}, \psi_{2},(N)}$ is an element of $M_{k}\left(\Gamma_{0}(N), \psi_{1} \psi_{2}\right)$.) It has $q$-expansion

$$
\begin{aligned}
& E_{k}^{\psi_{1}, \psi_{2},(N)}(q) \\
& :=-\delta_{\psi_{1}=1} L^{\left(N_{-} N_{0}\right)}\left(\psi_{2}, 0\right) L^{\left(N_{+} N_{0}\right)}\left(\psi_{1}, 1-k\right)+\sum_{n=1}^{\infty} \sigma_{k-1}^{\psi_{1}, \psi_{2},(N)}(n) q^{n} \\
& =-\delta_{\psi_{1}=1} \frac{B_{k, \psi_{1}}}{2 k} \prod_{\ell \mid N_{+}}\left(1-\psi_{1}(\ell) \ell^{k-1}\right) \prod_{\ell \mid N_{-}}\left(1-\psi_{2}(\ell)\right) \prod_{\ell \mid N_{0}}\left(1-\psi_{1}(\ell) \ell^{k-1}\right)\left(1-\psi_{2}(\ell)\right) \\
& \quad+\sum_{n=1}^{\infty} \sigma_{k-1}^{\psi_{1}, \psi_{2},(N)}(n) q^{n},
\end{aligned}
$$

where $L^{(N)}(\psi, s)$ denotes the $L$-function of a Dirichlet character $\psi$ with Euler factors at primes $\ell \mid N$ removed, and where

$$
\sigma_{k-1}^{\psi_{1}, \psi_{2},(N)}(n):=\sum_{\substack{0<d \mid n \\\left(d, N_{+}\right)=1 \\\left(n / d, N_{-}\right)=1 \\\left(n, N_{0}\right)=1}} \psi_{1}(n / d) \psi_{2}(d) d^{k-1} .
$$

Here, in keeping with our conventions, $\psi(m)=0$ if $(m, \mathfrak{f}(\psi)) \neq 1$. Then for $f \in S_{k}\left(\Gamma_{0}(N), \varepsilon_{f}\right)$ to have partial Eisenstein descent of type $\left(\psi_{1}, \psi_{2}, N_{+}, N_{-}, N_{0}\right)$ 
at $\lambda \mid p$ is equivalent to having

$$
\theta^{j} f(q) \equiv \theta^{j} E_{k}^{\psi_{1}, \psi_{2},(N)}(q)(\bmod \mathfrak{m})
$$

for all $j \geq 1$. When $f$ has full Eisenstein descent, this congruence holds for $j \geq 0$.

Remark 33. Suppose we are given $f \in S_{k}\left(\Gamma_{0}(N), \varepsilon_{f}\right)$ with partial Eisenstein descent of type $\left(\psi_{1}, \psi_{2}, N_{+}, N_{-}, N_{0}\right)$ over $M \bmod \mathfrak{m}$, in the sense of Definition 31 . In many situations, (5) is forced to hold a priori so that $f$ automatically has full Eisenstein descent. Suppose $\mathfrak{m} \neq \mathcal{O}_{M}$ (for otherwise, the conditions of Definition 31 are vacuous).

If $\psi_{1}$ is nontrivial, then $\delta_{\psi=1}=0$ and (5) holds. Now suppose that $\psi_{1}=1$. If $k$ is odd, then $B_{k, \psi_{1}}=B_{k}=0$, again forcing (5) to hold. Suppose $k$ is even. Let $\lambda \mid \mathfrak{m}$ be a prime ideal of residual characteristic not equal to 2 . By parts (3) and (1) of Theorem 34, we have $\psi_{1} \psi_{2}=\varepsilon_{f}$; in particular, we have $\psi_{2}(-1)=\varepsilon_{f}(-1)=$ $(-1)^{k}=1$. Hence $\psi_{2}$ is even, and so $B_{1, \psi_{2}}=0$ unless $\psi_{2}=1$. Now further suppose that $\psi_{2}=1$. Then (5) is still forced to hold unless $N_{-} N_{0}=1$. Now suppose $N_{-} N_{0}=1$. Let $\lambda \mid \mathfrak{m}$ be a prime ideal of residual characteristic $p$. Then conditions (1)-(4) still imply

$$
\theta f(q) \equiv \theta E_{k}^{\psi_{1}, \psi_{2},(N)}(q)(\bmod \lambda)
$$

Suppose $p>k+1$, so that, by [Serre 1973, Corollary 3, p. 326], $\theta$ is injective on mod- $\lambda$ modular forms. Then the above congruence implies

$$
f(q) \equiv E_{k}^{\psi_{1}, \psi_{2},(N)}(q)(\bmod \lambda) .
$$

Hence, if $\mathfrak{m}$ has order 0 or 1 at every prime of $\mathcal{O}_{M}$ and if the residual characteristic $p$ of every prime $\lambda \mid \mathfrak{m}$ satisfies $p>k+1$, then (5) is forced to hold. (See [Billerey and Menares 2013, Theorem 4.1] where a similar argument is given.) In particular,

$$
\frac{B_{k}}{2 k} \prod_{\ell \mid N_{+}}\left(1-\ell^{k-1}\right) \equiv 0(\bmod \mathfrak{m}) .
$$

When $k=2$, we have

$$
\frac{1}{24} \prod_{\ell \mid N_{+}}(1-\ell) \equiv 0(\bmod \mathfrak{m}),
$$

and therefore there exists at least one $\ell \mid N_{+}=N$ such that $\ell \equiv 1(\bmod \mathfrak{m})$, i.e., $\ell \equiv 1(\bmod \mathfrak{m} \cap \mathbb{Z})$.

Suppose we have a normalized eigenform $f(q)=\sum_{n=0}^{\infty} a_{n} q^{n} \in M_{k}\left(\Gamma_{0}(N), \varepsilon_{f}\right)$. Again let $M$ be a number field containing $E_{f}$. Let $k_{\lambda}$ denote the residue field of $\mathcal{O}_{M}$ at a prime $\lambda \mid p$. By a construction of Deligne [1971] we can attach a unique 
semisimple $p$-adic Galois representation $\rho_{f}: \operatorname{Gal}(\overline{\mathbb{Q}} / \mathbb{Q}) \rightarrow \mathrm{GL}_{2}\left(M_{\lambda}\right)$ unramified outside $p N$ and such that $\rho_{f}\left(\right.$ Frob $\left._{\ell}\right)$ has characteristic polynomial

$$
T^{2}-a_{\ell} T+\varepsilon_{f}(\ell) \ell^{k-1}
$$

for all $\ell \nmid p N$. Taking a $\operatorname{Gal}(\overline{\mathbb{Q}} / \mathbb{Q})$-stable lattice and the reduction mod $\lambda$, we get a representation $\bar{\rho}: \operatorname{Gal}(\overline{\mathbb{Q}} / \mathbb{Q}) \rightarrow \mathrm{GL}_{2}\left(k_{\lambda}\right)$ (whose semisimplification is independent of the choice of lattice). By Theorem 3 of [Atkin and Lehner 1970], we have $a_{\ell}= \pm \ell^{k / 2-1}$ for $\ell \| N$, and $a_{\ell}=0$ for $\ell^{2} \mid N$.

We have the following characterization of partial Eisenstein descent mod $\lambda$. See [Billerey and Menares 2013, Theorem 4.1] for a similar result to part (2) below. The elliptic curves case of part (1) was essentially done in [Serre 1972].

Theorem 34. Suppose $f \in S_{k}\left(\Gamma_{0}(N), \varepsilon_{f}\right)$ is a normalized newform, and define $\bar{\rho}_{f}: \operatorname{Gal}(\overline{\mathbb{Q}} / \mathbb{Q}) \rightarrow \mathrm{GL}_{2}\left(k_{\lambda}\right)$ to be (the semisimplification of ) the mod- $\lambda$ reduction of the associated semisimple Galois representation $\rho_{f}$. Then the following hold:

(1) Suppose $\bar{\rho}_{f}$ is reducible. Then $\bar{\rho}_{f} \cong k_{\lambda}\left(\tilde{\psi}_{1}\right) \oplus k_{\lambda}\left(\tilde{\psi}_{2} \omega^{k-1}\right)$, where we have $\tilde{\psi}_{i}=\psi_{i}(\bmod \lambda), i=1,2$, for some Dirichlet characters $\psi_{1}$ and $\psi_{2}$ over $\mathbb{Q}$ with $\psi_{1} \psi_{2}=\varepsilon_{f}$.

(2) Suppose $\bar{\rho}_{f}$ is reducible. Then, for all $\ell \| N$, either $a_{\ell} \equiv \psi_{1}(\ell)(\bmod \lambda)$ and $a_{\ell} \equiv \psi_{1}^{-1}(\ell) \ell^{k-2}(\bmod \lambda)$ and $\ell^{k-2} \equiv \psi_{1}^{2}(\ell)(\bmod \lambda)$, or $a_{\ell} \equiv \psi_{2}(\ell) \ell^{k-1}$ $(\bmod \lambda)$ and $a_{\ell} \equiv \psi_{2}^{-1}(\ell) \ell^{-1}(\bmod \lambda)$ and $\ell^{k} \equiv \psi_{2}^{-2}(\ell)(\bmod \lambda)$.

(3) Let $N_{+}$denote any product of all primes $\ell \| N$ satisfying $a_{\ell} \equiv \psi_{1}(\ell)(\bmod \lambda)$ and $N_{-}$any product of $\ell \| N$ satisfying $a_{\ell} \equiv \psi_{2}(\ell) \ell^{k-1}(\bmod \lambda)$, such that $N_{+} N_{-}$is the squarefree part of $N$ (so that, in particular, $\left(N_{+}, N_{-}\right)=1$ ). Let $N_{0}$ be the squarefull part of $N$. Then $\bar{\rho}_{f}$ is reducible if and only if $f$ has partial Eisenstein descent of type $\left(\psi_{1}, \psi_{2}, N_{+}, N_{-}, N_{0}\right)$ over $M_{\lambda} \bmod \lambda$.

Proof. (1) Since $\bar{\rho}_{f}$ is reducible and semisimple, we can write $\bar{\rho}_{f}=k_{\lambda}\left(\chi_{1}\right) \oplus k_{\lambda}\left(\chi_{2}\right)$, where $\chi_{1}, \chi_{2}: \operatorname{Gal}(\overline{\mathbb{Q}} / \mathbb{Q}) \rightarrow k_{\lambda}^{\times}$. Hence $\chi_{1} \chi_{2}=\operatorname{det}\left(\bar{\rho}_{f}\right)=\varepsilon_{f} \omega^{k-1}$, and so for our statement it suffices to show that one of $\chi_{1}, \chi_{2}$ is unramified outside the squarefull part of $N$ (since $\mathfrak{f}\left(\varepsilon_{f}\right)$ divides the squarefull part of $N$ ). Since $\bar{\rho}_{f}$ is unramified outside $p N$, clearly both $\chi_{1}, \chi_{2}$ are unramified outside $p N$. For $\ell \| N$, the local representation $\bar{\rho}_{f, \ell}$ (i.e., the restriction of $\bar{\rho}_{f, \ell}$ to the decomposition group $\operatorname{Gal}\left(\overline{\mathbb{Q}}_{\ell} / \mathbb{Q}_{\ell}\right)$ ) has conductor $\ell$. Thus the corresponding automorphic representation $\pi_{\ell}$ of $\rho_{\ell}$ has conductor $\ell$, and so, by the classification of admissible representations of $\mathrm{GL}_{2}$ over local fields (see [Gelbart 1975, p. 73]), at most one of $\chi_{1}$ and $\chi_{2}$ is ramified at $\ell$. Thus $\chi_{1}, \chi_{2}$ are unramified inside the squarefree part of $N$. Finally, since $\bar{\rho}_{f, p}$ is reducible, by a theorem of Deligne (see [Gross 1990, Introduction]), $a_{p}$ is not 
congruent to $0 \bmod \lambda$ and $\bar{\rho}_{f, p}$ is of the form

$$
\left(\begin{array}{cc}
\omega^{k-1} \mu_{p}\left(\varepsilon_{f}(p) / a_{p}\right) & * \\
0 & \mu_{p}\left(a_{p}\right)
\end{array}\right)
$$

where $\mu_{p}(\alpha)$ is the unramified character of $\operatorname{Gal}\left(\overline{\mathbb{Q}}_{p} / \mathbb{Q}_{p}\right)$ taking Frob $p$ to $\alpha$. Hence exactly one of $\chi_{1}, \chi_{2}$ is ramified at $p$.

Putting this all together, we see that $\left\{\chi_{1}, \chi_{2}\right\}=\left\{\psi_{1} \bmod \lambda, \psi_{2} \omega^{k-1} \bmod \lambda\right\}$ for some Dirichlet characters $\psi_{1}$ and $\psi_{2}$ with $\psi_{1} \psi_{2}=\varepsilon_{f}$, and so we are done.

(2) It is a theorem of Langlands (see [Loeffler and Weinstein 2012, Proposition 2.8]) that, for $\ell \| N, \bar{\rho}_{f, \ell}$ is of the form

$$
\left(\begin{array}{cc}
\omega_{\ell}^{k / 2} \mu_{\ell}\left(\varepsilon_{f}(\ell) \ell^{k / 2-1} / a_{\ell}\right) & * \\
0 & \omega_{\ell}^{k / 2-1} \mu_{\ell}\left(a_{\ell} / \ell^{k / 2-1}\right)
\end{array}\right)
$$

where $\omega_{\ell}$ is the localization of $\omega$ to $\ell$. Thus by (1), we have

$$
\left\{\psi_{1, \ell} \bmod \lambda, \psi_{2, \ell} \omega_{\ell}^{k-1} \bmod \lambda\right\}=\left\{\omega_{\ell}^{k / 2} \mu_{\ell}\left(\varepsilon_{f}(\ell) \ell^{k / 2-1} / a_{\ell}\right), \omega_{\ell}^{k / 2} \mu_{\ell}\left(a_{\ell} / \ell^{k / 2-1}\right)\right\} .
$$

Note that $\mu_{\ell}\left(\ell^{k / 2-1} / a_{\ell}\right)$ is a quadratic character.

Suppose first that $\psi_{1, \ell} \equiv \omega_{\ell}^{k / 2} \mu_{\ell}\left(\varepsilon_{f}(\ell) \ell^{k / 2-1} / a_{\ell}\right)(\bmod \lambda)$, and that $\psi_{2, \ell} \omega_{\ell}^{k-1} \equiv$ $\omega_{\ell}^{k / 2-1} \mu_{\ell}\left(\ell^{k / 2-1} / a_{\ell}\right)(\bmod \lambda)$. Plugging in Frob ${ }_{\ell}$ to both congruences, the first congruence implies $a_{\ell} \equiv \psi_{2, \ell}(\ell) \ell^{k-1}=\psi_{2}(\ell) \ell^{k-1}(\bmod \lambda)$, and the second congruence implies $a_{\ell} \equiv \psi_{2, \ell}^{-1}(\ell) \ell^{-1}=\psi_{2}^{-1}(\ell) \ell^{-1}(\bmod \lambda)$.

Suppose next that $\psi_{1, \ell} \equiv \omega_{\ell}^{k / 2-1} \mu_{\ell}\left(a_{\ell} / \ell^{k / 2-1}\right)(\bmod \lambda)$, and that $\psi_{2, \ell} \omega_{\ell}^{k-1} \equiv$ $\omega_{\ell}^{k / 2} \mu_{\ell}\left(\varepsilon_{f}(\ell) \ell^{k / 2-1} / a_{\ell}\right)(\bmod \lambda)$. Plugging in Frob $_{\ell}$ to both congruences, the first congruence implies both $a_{\ell} \equiv \psi_{1, \ell}(\ell)=\psi_{1}(\ell)(\bmod \lambda)$ and $a_{\ell} \equiv \psi_{1, \ell}^{-1}(\ell) \ell^{k-2}=$ $\psi_{1}^{-1}(\ell) \ell^{k-2}(\bmod \lambda)\left(\right.$ we get both since we have $\left.\mu_{\ell}\left(a_{\ell} / \ell^{k / 2-1}\right)=\mu_{\ell}^{-1}\left(a_{\ell} / \ell^{k / 2-1}\right)\right)$, and the second congruence gives no new congruences.

(3) For $\ell \nmid p N$ we have $a_{\ell}=\operatorname{trace}\left(\bar{\rho}_{f}\right)\left(\operatorname{Frob}_{\ell}\right) \equiv \psi_{1}(\ell)+\psi_{2}(\ell) \ell^{k-1}(\bmod \lambda)$. For $\ell \mid N_{+}$we have $a_{\ell} \equiv \psi_{1}(\ell)(\bmod \lambda)$, for $\ell \mid N_{-}$we have $a_{\ell} \equiv \psi_{2}(\ell) \ell^{k-1}$ $(\bmod \lambda)$, and for $\ell \mid N_{0}$ we have $a_{\ell} \equiv 0(\bmod \lambda)$. Finally, by the Cebotarev density theorem and continuity of $\operatorname{trace}\left(\bar{\rho}_{f}\right)$, we have $a_{p}=\operatorname{trace}\left(\bar{\rho}_{f}\left(\operatorname{Frob}_{p}\right)\right) \equiv$ $\psi_{1}(p)+\psi_{2}(p) p^{k-1} \equiv \psi_{1}(p)(\bmod \lambda)$. Hence $f$ has partial Eisenstein descent of type $\left(\psi_{1}, \psi_{2}, N_{+}, N_{-}, N_{0}\right)$ at $\lambda \mid p$.

If $f$ has partial Eisenstein descent of type $\left(\psi_{1}, \psi_{2}, N_{+}, N_{-}, N_{0}\right)$ at $\lambda \mid p$, then for $\ell \nmid N$ we have $\operatorname{trace}\left(\bar{\rho}_{f}\right)\left(\operatorname{Frob}_{\ell}\right) \equiv \psi_{1}(\ell)+\psi_{2}(\ell) \ell^{k-1}(\bmod \lambda)$. Hence if $\bar{\rho}:=$ $k_{\lambda}\left(\psi_{1}\right) \oplus k_{\lambda}\left(\psi_{2} \omega^{k-1}\right)$, then $\operatorname{trace}\left(\bar{\rho}_{f}\right)\left(\operatorname{Frob}_{\ell}\right) \equiv \operatorname{trace}(\bar{\rho})\left(\operatorname{Frob}_{\ell}\right)$ for all $\ell \nmid N$. Thus by the Cebotarev density theorem and continuity of trace, $\operatorname{trace}\left(\bar{\rho}_{f}\right)(g) \equiv \operatorname{trace}(\bar{\rho})(g)$ for all $g \in \operatorname{Gal}(\overline{\mathbb{Q}} / \mathbb{Q})$. Hence, by the Brauer-Nesbitt theorem, $\bar{\rho}_{f} \cong \bar{\rho}$.

For $k=2$ and $M=E_{f}=\mathbb{Q}$, we note the following corollary. 
Theorem 35. Suppose $E / \mathbb{Q}$ is an elliptic curve and $p$ is a prime such that $E[p]$ is a reducible mod-p Galois representation. Then the associated normalized newform $f_{E} \in S_{2}\left(\Gamma_{0}(N)\right)$ has partial Eisenstein descent over $\mathbb{Q}_{p} \bmod p \mathbb{Z}_{p}$.

\section{Proof of the main theorem}

First, we examine complex $L$-values arising from twisted traces of Eisenstein series evaluated at CM points, analogous to such traces for normalized newforms interpolated by Bertolini, Darmon, and Prasanna's $p$-adic $L$-function (see Theorem 24). Parts of the calculation in Section 4.1 are implicit in [Hida and Tilouine 1993], but for our purposes which require explicit identities, and for the sake of completeness, we include the full calculation here.

4.1. Twisted traces of Eisenstein series over CM points. Let $k \geq 2$ be an integer, and let $\psi_{1}, \psi_{2}$ be two Dirichlet characters over $\mathbb{Q}$ with conductors $u$ and $t$, respectively. (Here and throughout this section, for simplicity, we identify these ideals in $\mathbb{Z}$ with their unique positive rational integer generators.) We also assume that $u t=N^{\prime}$ (where $u$ and $t$ are not necessarily coprime), and that $\left(\psi_{1} \psi_{2}\right)(-1)=(-1)^{k}$. Recall our Eisenstein series (see Remark 32)

$$
E_{k}^{\psi_{1}, \psi_{2}}(\tau):=\delta_{\psi_{1}=1} \frac{1}{2} L\left(\psi_{1}, 1-k\right)+\sum_{n=1}^{\infty} \sigma_{k-1}^{\psi_{1}, \psi_{2}}(n) q^{n},
$$

where $q=e^{2 \pi i \tau}$ and

$$
\sigma_{k-1}^{\psi_{1}, \psi_{2}}(n)=\sum_{0<d \mid n} \psi_{1}(n / d) \psi_{2}(d) d^{k-1}
$$

Note that the nebentypus of $E_{k}^{\psi_{1}, \psi_{2}}$ is $\varepsilon_{E_{k}, \psi_{2}}=\psi_{1} \psi_{2}$.

Recalling the Maass-Shimura derivative $\partial$ defined in Section 3.1, one checks by direct computation that

$$
\partial^{j} E_{k}^{\psi_{1}, \psi_{2}}(\tau)=\frac{t^{k} \Gamma(k+j)}{2(2 \pi i)^{k+j} \mathfrak{g}\left(\psi_{2}^{-1}\right)} \sum_{c=0}^{u-1} \sum_{d=0}^{t-1} \sum_{e=0}^{u-1} \sum_{\substack{(m, n) \equiv \\(c t, d+e t)\left(N^{\prime}\right)}} \frac{\psi_{1}(c) \psi_{2}^{-1}(d)}{(m \tau+n)^{k+2 j}}\left(\frac{|m \tau+n|^{2}}{\tau-\bar{\tau}}\right)^{j},
$$

with the last sum over $(m, n) \in \mathbb{Z}^{2} \backslash\{0\}$ satisfying $(m, n) \equiv(c t, d+e t)\left(\bmod N^{\prime}\right)$.

Recall that under our Assumptions $1, D_{K}$ is taken to be odd. (The calculations for the even case are entirely analogous to those of the odd case, but we do not explicitly write them out here.) Given an integral primitive (i.e., having no rational integral divisors other than \pm 1 ) ideal $\mathfrak{a}$ of $\mathcal{O}_{K}$, we can write

$$
\mathfrak{a}=\mathbb{Z} \frac{b+\sqrt{D_{K}}}{2}+\mathbb{Z} a,
$$


where $a=\left|\mathrm{Nm}_{K / \mathbb{Q}}(\mathfrak{a})\right|$ and $b^{2}-4 a c=D_{K}$; the triple $(a,-b, c)$ determines the primitive positive definite binary quadratic form associated with the ideal class of $\mathfrak{a}$. We set

$$
\tau_{\mathfrak{a}}:=\frac{b+\sqrt{D_{K}}}{2 a}
$$

so that $\tau_{\mathfrak{a}} \in \mathcal{H}^{+}$is the root of the dehomogenized quadratic form $a \tau^{2}-b \tau+c=0$ with positive imaginary part. We call $\tau_{\mathfrak{a}}$ a $C M$ point. Note that $\left\langle\tau_{\mathfrak{a}}, 1\right\rangle$ generates $\overline{\mathfrak{a}}^{-1}$. (Here, for $\tau \in \mathcal{H}^{+}$, we have $\langle\tau, 1\rangle=\mathbb{Z} \tau+\mathbb{Z}$.)

Suppose that $K$ satisfies the Heegner hypothesis with respect to $N^{\prime}$. Fix an ideal $\mathfrak{N}^{\prime}$ such that $\mathcal{O}_{K} / \mathfrak{N}^{\prime}=\mathbb{Z} / N^{\prime}$, and write

$$
\mathfrak{N}^{\prime}=\mathbb{Z} \frac{b_{N^{\prime}}+\sqrt{D_{K}}}{2}+\mathbb{Z} N^{\prime}, \quad \mathfrak{a} \mathfrak{N}^{\prime}=\mathbb{Z} \frac{b_{a N^{\prime}}+\sqrt{D_{K}}}{2}+\mathbb{Z} a N^{\prime},
$$

where $b_{a N^{\prime}}^{2}-4 a N^{\prime} c=D_{K}$ for some $c \in \mathbb{Z}$. Write $\mathfrak{N}^{\prime}=\mathfrak{u t}$, where $\mathcal{O}_{K} / \mathfrak{u}=\mathbb{Z} / u$ and $\mathcal{O}_{K} / \mathfrak{t}=\mathbb{Z} / t$.

Suppose we have a Dirichlet character $\phi:\left(\mathbb{Z} / N^{\prime}\right)^{\times} \rightarrow \mathbb{C}^{\times}$. By the identification $\mathcal{O}_{K} / \mathfrak{N}^{\prime}=\mathbb{Z} / N^{\prime}$, we can view $\phi$ as a character $\phi: \mathbb{A}_{K}^{\times, \mathfrak{N}^{\prime}} \rightarrow \prod_{v \mid \mathfrak{N}^{\prime}}\left(\mathcal{O}_{K, v} / \mathfrak{N}^{\prime} \mathcal{O}_{K, v}\right)^{\times} \cong$ $\left(\mathcal{O}_{K} / \mathfrak{N}^{\prime}\right)^{\times} \rightarrow \mathbb{C}^{\times}$, where $\mathbb{A}_{K}^{\times, \mathfrak{N}^{\prime}}$ denotes the idèles prime to $\mathfrak{N}^{\prime}$; when we view $\phi$ in this way, we will write $\phi\left(x \bmod \mathfrak{N}^{\prime}\right)$ for its value at $x \in \mathbb{A}_{K}^{\times, \mathfrak{N}^{\prime}}$. Given a Hecke character $\chi$ over $K$ of finite type $\left(\mathfrak{N}^{\prime}, \phi\right)$ and infinity type $\left(j_{1}, j_{2}\right)$, recall that the associated Grossencharacter on ideals $\mathfrak{a}$ prime to $\mathfrak{N}^{\prime}$ is given by

$$
\chi(\mathfrak{a})=\chi(x) \phi\left(x \bmod \mathfrak{N}^{\prime}\right) x_{\infty}^{j_{1}} \bar{x}_{\infty}^{j_{2}},
$$

where $x \in \mathbb{A}_{K}^{\times, \mathfrak{N}^{\prime}}$ is such that $\operatorname{ord}_{v}(x)=\operatorname{ord}_{v}(\mathfrak{a})$ for all finite places $v$. We consider the twisted trace

$$
\sum_{[\mathfrak{a}] \in \operatorname{Cl}\left(\mathcal{O}_{K}\right)}\left(\chi_{j}\right)^{-1}\left(\overline{\mathfrak{a} \mathfrak{N}^{\prime}}\right) \partial^{j} E_{k}^{\psi_{1}, \psi_{2}}\left(\tau_{\mathfrak{a} \mathfrak{N}^{\prime}}\right)
$$

where $\mathfrak{a}$ ranges over a set of primitive integral ideal representatives of $\operatorname{Cl}\left(\mathcal{O}_{K}\right)$ chosen to be prime to $N^{\prime}$, and where $\chi$ is of infinity type $(k+j,-j)$ and of finite type $\left(\mathfrak{N}^{\prime}, \psi_{1} \psi_{2}\right)$ (so that the above summands depend only on the ideal classes of the $\left.\overline{\mathfrak{a} \mathfrak{N}^{\prime}}\right)$.

Suppose $\alpha=m\left(b_{a N^{\prime}}+\sqrt{\overline{D_{K}}}\right) /(2 a u t)+n \in(\overline{\mathfrak{a} \mathfrak{u}})^{-1}$ with $(m, n) \equiv(c t, d+e t)$ $\left(\bmod N^{\prime}\right)$. Then $a u \alpha=m\left(b_{a N^{\prime}}+\sqrt{D_{K}}\right) /(2 t)+a u n \in \mathfrak{a u} \subset \mathcal{O}_{K}$ is mapped to $b_{a N^{\prime}} c \in \mathbb{Z} / u$ under the identification $\mathcal{O}_{K} / \overline{\mathfrak{u}}=\mathbb{Z} / u$. We see this by writing $m=c t+q N^{\prime}$ for some $q \in \mathbb{Z}$ and

$$
\begin{aligned}
a u \alpha & =\frac{m b_{a N^{\prime}}}{t}-m \frac{b_{a N^{\prime}}-\sqrt{D_{K}}}{2}+a u n \\
& =b_{a N^{\prime}} c-m \frac{b_{a N^{\prime}}-\sqrt{D_{K}}}{2}+q u \equiv b_{a N^{\prime}} c(\bmod \overline{\mathfrak{u}}) .
\end{aligned}
$$


We thus have $\psi_{1}((a u \alpha) \bmod \overline{\mathfrak{u}})=\psi_{1}\left(b_{a N^{\prime}} c\right)$. Since $b_{a N^{\prime}}+\sqrt{D_{K}} \in \mathfrak{a} \mathfrak{N}^{\prime}$, in particular we have $b_{a N^{\prime}} \equiv-\sqrt{D_{K}}(\bmod \mathfrak{u})$, meaning $\psi_{1}\left(b_{a N^{\prime}}\right)=\psi_{1}\left(-\sqrt{D_{K}} \bmod \mathfrak{u}\right)$; henceforth we will write $\psi_{1}\left(-\sqrt{D_{K}}\right)=\psi_{1}\left(-\sqrt{D_{K}} \bmod \mathfrak{u}\right)$ for simplicity. On the other hand, note that $\alpha$ is mapped to $d \in \mathbb{Z} / t$ under the isomorphism $(\overline{\mathfrak{a} \mathfrak{u}})^{-1} /(\overline{\mathfrak{a u}})^{-1} \mathfrak{t} \cong$ $\mathcal{O}_{K} / \mathfrak{t}=\mathbb{Z} / t$. Thus, $\psi_{2}(\overline{\mathfrak{a} \mathfrak{u}}(\alpha) \bmod \mathfrak{t})=\psi_{2}(d)$ (since $\overline{\mathfrak{a} \mathfrak{u}}$ is prime to $\left.\mathfrak{t}\right)$. In all,

$$
\begin{aligned}
& \frac{\psi_{1}(c) \overline{\psi_{2}}(d)}{\left(m \tau_{\mathfrak{a} \mathfrak{N}^{\prime}}+n\right)^{k+2 j}}\left(\frac{\left|m \tau_{\mathfrak{a} \mathfrak{N}^{\prime}}+n\right|^{2}}{\left.\tau_{\mathfrak{a} \mathfrak{N}^{\prime}}-\overline{\tau_{\mathfrak{a} \mathfrak{N}^{\prime}}}\right)^{j}}\right. \\
& \quad=\frac{\psi_{1}^{-1}\left(-\sqrt{D_{K}}\right) \psi_{1}((a u \alpha) \bmod \overline{\mathfrak{u}}) \psi_{2}^{-1}(\overline{\mathfrak{a} \mathfrak{u}}(\alpha) \bmod \mathfrak{t})}{\alpha^{k+2 j}}\left(\frac{\operatorname{Nm}_{K / \mathbb{Q}}\left(\overline{\mathfrak{a} \mathfrak{N}^{\prime}}(\alpha)\right)}{\sqrt{D_{K}}}\right)^{j} .
\end{aligned}
$$

Suppose $k>2$. Then we can rewrite each summand in the twisted trace as $\left(\chi_{j}\right)^{-1}\left(\overline{\mathfrak{a N N}^{\prime}}\right) \partial^{j} E_{k}^{\psi_{1}, \psi_{2}}\left(\tau_{\mathfrak{a} \mathfrak{N}^{\prime}}\right)$

$$
\begin{aligned}
& =\frac{t^{k} \Gamma(k+j) \psi_{1}^{-1}\left(-\sqrt{D_{K}}\right)}{2(2 \pi i)^{k+j} \mathfrak{g}\left(\psi_{2}^{-1}\right)}\left(\chi_{j}\right)^{-1}\left(\overline{\mathfrak{a} \mathfrak{N}^{\prime}}\right) \\
& \times \sum_{\alpha \in\left(\overline{\mathfrak{a N}^{\prime}}\right)^{-1}} \frac{\psi_{1}((\text { au } \alpha) \bmod \overline{\mathfrak{u}}) \psi_{2}^{-1}(\overline{\mathfrak{a} \mathfrak{u}}(\alpha) \bmod \mathfrak{t})}{\alpha^{k+2 j}}\left(\frac{\left|\mathrm{Nm}_{K / \mathbb{Q}}\left(\overline{\mathfrak{a} \mathfrak{N}^{\prime}}(\alpha)\right)\right|}{\sqrt{D_{K}}}\right)^{j} \\
& \left(\overline{\mathfrak{a} \mathfrak{N}^{\prime}}(\alpha), \overline{\mathfrak{u}} \mathfrak{N}^{\prime}\right)=1
\end{aligned}
$$

$$
\begin{aligned}
& =\frac{t^{k} \Gamma(k+j) \psi_{1}^{-1}\left(-\sqrt{D_{K}}\right)}{2(2 \pi i)^{k+j} \mathfrak{g}\left(\psi_{2}^{-1}\right){\sqrt{D_{K}}}^{j}} \frac{\chi^{-1}(\overline{\mathfrak{t}})\left(\chi_{-k / 2}\right)^{-1}(\overline{\mathfrak{a} \mathfrak{u}})}{\left|\mathrm{Nm}_{K / \mathbb{Q}}(\overline{\mathfrak{a} \mathfrak{u}})\right|^{k / 2}} \\
& \quad \times \sum_{\substack{\alpha \in(\overline{\mathfrak{a u}})^{-1} \\
\left(\overline{\mathfrak{a} \mathfrak{u}}(\alpha), \overline{\mathfrak{u}} \mathfrak{T}^{\prime}\right)=1}}\left(\frac{\bar{\alpha}}{\alpha}\right)^{k / 2+j} \frac{\psi_{1}((a u \alpha) \bmod \overline{\mathfrak{u}}) \psi_{2}^{-1}(\overline{\mathfrak{a} \mathfrak{u}}(\alpha) \bmod \mathfrak{t})}{\left|\operatorname{Nm}_{K / \mathbb{Q}}(\alpha)\right|^{k / 2}}
\end{aligned}
$$$$
=\frac{t^{k} \Gamma(k+j) \psi_{1}^{-1}\left(-\sqrt{D_{K}}\right) \chi^{-1}(\overline{\mathfrak{t}})}{2(2 \pi i)^{k+j} \mathfrak{g}\left(\psi_{2}^{-1}\right){\sqrt{D_{K}}}^{j}}
$$$$
\times \sum\left(\chi_{-k / 2}\right)^{-1}(\overline{\mathfrak{a} u}(\alpha)) \psi_{1}(\overline{\mathfrak{a} \mathfrak{u}}(\alpha) \bmod \mathfrak{u}) \psi_{2}(\overline{\mathfrak{a} u}(\alpha) \bmod \mathfrak{t})
$$$$
\alpha \in(\overline{\mathfrak{a u}})^{-1}
$$$$
\left(\overline{\mathfrak{a u}}(\alpha), \overline{\mathrm{u}} \mathfrak{N}^{\prime}\right)=1
$$

$$
\begin{aligned}
& \times \frac{\psi_{1}((\text { au } \alpha) \bmod \overline{\mathfrak{u}}) \psi_{2}^{-1}(\overline{\mathfrak{a} \mathfrak{u}}(\alpha) \bmod \mathfrak{t})}{\left|\mathrm{Nm}_{K / \mathbb{Q}}(\overline{\mathfrak{a} \mathfrak{u}}(\alpha))\right|^{k / 2}} \\
& =\frac{t^{k} \Gamma(k+j) \psi_{1}^{-1}\left(-\sqrt{D_{K}}\right) \chi^{-1}(\overline{\mathfrak{t}})}{(2 \pi i)^{k+j} \mathfrak{g}\left(\psi_{2}^{-1}\right){\sqrt{D_{K}}}^{j}} \\
& \times \sum_{\substack{\alpha \in(\overline{\mathfrak{a} u})^{-1} \\
\left(\overline{\mathfrak{a} u}(\alpha), \overline{\mathfrak{u}} \mathfrak{N}^{\prime}\right)=1}}\left(\chi_{-k / 2}\right)^{-1}(\overline{\mathfrak{a} \mathfrak{u}}(\alpha)) \frac{\psi_{1}\left(\left(a u \operatorname{Nm}_{K / \mathbb{Q}}(\alpha)\right) \bmod \mathfrak{u}\right)}{\left|\operatorname{Nm}_{K / \mathbb{Q}}(\overline{\mathfrak{a} \mathfrak{u}}(\alpha))\right|^{k / 2}} \\
& =\frac{\left|\mathcal{O}_{K}^{\times}\right|}{2} \frac{t^{k} \Gamma(k+j) \psi_{1}^{-1}\left(-\sqrt{D_{K}}\right) \chi^{-1}(\overline{\mathfrak{t}})}{(2 \pi i)^{k+j} \mathfrak{g}\left(\psi_{2}^{-1}\right){\sqrt{D_{K}}}^{j}} \sum_{\substack{\mathfrak{b} \subset \mathcal{O}_{K} \\
[\mathfrak{b}]=\left[\cos _{\mathfrak{a} \mathfrak{u}]}\right] \in \operatorname{Cl}\left(\mathcal{O}_{K}\right)}} \frac{\left(\psi_{1 / K}\left(\chi_{-k / 2}\right)^{-1}\right)(\mathfrak{b})}{\left|\operatorname{Nm}_{K}(\mathfrak{b})\right|^{k} / 2} . \\
& \left(\mathfrak{b}, \overline{\mathfrak{u}} \mathfrak{N}^{\prime}\right)=1
\end{aligned}
$$


The penultimate equality is justified since $\psi_{1}((a u \alpha) \bmod \overline{\mathfrak{u}})=\psi_{1}(\overline{\mathfrak{a} \mathfrak{u}}(\alpha) \bmod \overline{\mathfrak{u}})=$ $\psi_{1}(\mathfrak{a} \mathfrak{u}(\bar{\alpha}) \bmod \mathfrak{u})($ the first equality here follows since $\mathfrak{a} \mathfrak{u}$ is prime to $\overline{\mathfrak{u}})$. The factor of $\left|\mathcal{O}_{K}^{\times}\right|$appears because any integral ideal $\mathfrak{b} \subset \mathcal{O}_{K}$ can be written as $\overline{\mathfrak{a u}}(\alpha)$ for some ideal class representative $\mathfrak{a}$ and some element $\alpha \in(\overline{\mathfrak{a} u})^{-1}$ determined uniquely up to an element of $\mathcal{O}_{K}^{\times}=\{ \pm 1\}$. Since $\left(\psi_{1} \psi_{2}\right)(-1)=(-1)^{k}=(-1)^{k+2 j}$, we see that each summand of the second line has the same value for $\alpha$ or $-\alpha$. Now since $\left|\mathcal{O}_{K}^{\times}\right|=2$, we finally have, after summing both sides of the above equality over all our ideal class representatives $\mathfrak{a}$,

$\sum_{[\mathfrak{a} \in \in \mathcal{O}}\left(\chi_{j}\right)^{-1}\left(\overline{\mathfrak{a} \mathfrak{N}^{\prime}}\right) \partial^{j} E_{k}^{\psi_{1}, \psi_{2}}\left(\tau_{\mathfrak{a} \mathfrak{N}^{\prime}}\right)$

$[\mathfrak{a}] \in \operatorname{Cl}\left(\mathcal{O}_{K}\right)$

$$
=\frac{t^{k} \Gamma(k+j) \psi_{1}^{-1}\left(-\sqrt{D_{K}}\right) \chi^{-1}(\overline{\mathfrak{t}})}{(2 \pi i)^{k+j} \mathfrak{g}\left(\psi_{2}^{-1}\right){\sqrt{D_{K}}}^{j}} L\left(\psi_{1 / K}\left(\chi_{-k / 2}\right)^{-1}, \frac{k}{2}\right) .
$$

For $k=2$ note that, by the same calculation as above, we have, for all $s \in \mathbb{C}$ with $\Re(s)>0$,

$\sum\left(\chi_{j}\right)^{-1}\left(\overline{\mathfrak{a} \mathfrak{N}^{\prime}}\right)$

$[\mathfrak{a}] \in \operatorname{Cl}\left(\mathcal{O}_{K}\right)$

$$
\sum_{\alpha \in(\overline{\mathfrak{a u}})^{-1}} \frac{\psi_{1}^{-1}\left(-\sqrt{D_{K}}\right) \psi_{1}(\overline{\mathfrak{a}}(a u \alpha) \bmod \overline{\mathfrak{u}}) \psi_{2}^{-1}(\overline{\mathfrak{a} \mathfrak{u}}(\alpha) \bmod \mathfrak{t})}{\alpha^{2+2 j}}\left(\frac{\left|\operatorname{Nm}_{K} / \mathbb{Q}\left(\overline{\mathfrak{a} \mathfrak{N}^{\prime}}(\alpha)\right)\right|}{\sqrt{D_{K}}}\right)^{j-s}
$$

$\left(\overline{\mathfrak{a u}}(\alpha), \overline{\mathrm{u}} \mathfrak{N}^{\prime}\right)=1$

$$
=\frac{\psi_{1}^{-1}\left(-\sqrt{D_{K}}\right) \chi^{-1}(\overline{\mathfrak{t}})}{{\sqrt{D_{K}}}^{j-s} t^{s}} L\left(\psi_{1 / K}\left(\chi_{-1}\right)^{-1}, 1+s\right) .
$$

Note that each summand of the inner sum can be written as the special value of a real analytic Eisenstein series at a certain CM point:

$$
\sum_{c=0}^{u-1} \sum_{d=0}^{t-1} \sum_{e=0}^{u-1} \sum_{\begin{array}{c}
(m, n) \in \mathbb{Z}^{2} \backslash\{0\} \\
(m, n) \equiv \\
(c t, d+e t)\left(N^{\prime}\right)
\end{array}} \frac{\psi_{1}(c) \psi_{2}^{-1}(d)}{\left(m \tau_{\mathfrak{a} \mathfrak{N}^{\prime}}+n\right)^{2+2 j}}\left(\frac{\left|m \tau_{\mathfrak{a} \mathfrak{N}^{\prime}}+n\right|^{2}}{\tau_{\mathfrak{a} \mathfrak{N}^{\prime}}-\overline{\tau_{\mathfrak{a} \mathfrak{N}^{\prime}}}}\right)^{j-s} .
$$

Using standard analytic arguments, one can show that the above expression tends to $\partial^{j} E_{2}^{\psi_{1}, \psi_{2}}\left(\tau_{\mathfrak{a} \mathfrak{N}^{\prime}}\right)$ as $s \rightarrow 0$. Hence, combining the above, we have

$$
\begin{aligned}
\sum_{[\mathfrak{a}] \in \operatorname{Cl}\left(\mathcal{O}_{K}\right)}\left(\chi_{j}\right)^{-1}\left(\overline{\mathfrak{a} \mathfrak{N}^{\prime}}\right) \partial^{j} E_{2}^{\psi_{1}, \psi_{2}}\left(\tau_{\left.\mathfrak{a} \mathfrak{\mathfrak { N } ^ { \prime }}\right)}\right. & \\
& =\lim _{s \rightarrow 0} \frac{t^{2} \Gamma(2+j) \psi_{1}^{-1}\left(-\sqrt{D_{K}}\right) \chi^{-1}(\overline{\mathfrak{t}})}{(2 \pi i)^{2+j} \mathfrak{g}\left(\psi_{2}^{-1}\right){\sqrt{D_{K}}}^{j-s} t^{s}} L\left(\psi_{1 / K}\left(\chi_{-1}\right)^{-1}, 1+s\right) \\
& =\frac{t^{2} \Gamma(2+j) \psi_{1}^{-1}\left(-\sqrt{D_{K}}\right) \chi^{-1}(\overline{\mathfrak{t}})}{(2 \pi i)^{2+j} \mathfrak{g}\left(\psi_{2}^{-1}\right){\sqrt{D_{K}}}^{j}} L\left(\psi_{1 / K}\left(\chi_{-1}\right)^{-1}, 1\right) .
\end{aligned}
$$


Proposition 36. Let $k \geq 2$ be an integer. Suppose $\chi$ is of infinity type $(k+j,-j)$ and $\chi_{-k / 2}$ has trivial central character and is of finite type $\left(\mathfrak{N}^{\prime}, \psi_{1} \psi_{2}\right)$. Suppose also that $\psi_{1}$ and $\psi_{2}$ are Dirichlet characters over $\mathbb{Q}$ with conductors $u$ and $t$, respectively, such that $u t=N$ and $\left(\psi_{1} \psi_{2}\right)(-1)=(-1)^{k}$. Then

$$
\sum_{[\mathfrak{a}] \in \operatorname{Cl}\left(\mathcal{O}_{K}\right)}\left(\chi_{j}\right)^{-1}(\mathfrak{a}) \partial^{j} E_{k}^{\psi_{1}, \psi_{2}}\left(\tau_{\mathfrak{a}}\right)=\frac{t^{k} \Gamma(k+j) \psi_{1}^{-1}\left(-\sqrt{D_{K}}\right) \chi^{-1}(\overline{\mathfrak{t}})}{(2 \pi i)^{k+j} \mathfrak{g}\left(\psi_{2}^{-1}\right){\sqrt{D_{K}}}^{j}} L\left(\psi_{1 / K} \chi^{-1}, 0\right) .
$$

4.2. Stabilization operators. We have the following "stabilization operators" acting on normalized $\Gamma_{0}\left(N^{\prime}\right)$-eigenforms $F$ with character $\varepsilon_{F}$. Given a rational prime $\ell \nmid N^{\prime}$, let $a_{\ell}$ denote the eigenvalue of $F$ under the Hecke operator $T_{\ell}$, let $\left(\alpha_{\ell}, \beta_{\ell}\right)$ denote (some henceforth fixed ordering of) the algebraic numbers such that $\alpha_{\ell}+\beta_{\ell}=a_{\ell}$, $\alpha_{\ell} \beta_{\ell}=\ell^{k-1} \varepsilon_{F}(\ell)$, and $\operatorname{ord}_{\ell}\left(\alpha_{\ell}\right) \leq \operatorname{ord}_{\ell}\left(\beta_{\ell}\right)$. Now define

$$
\begin{aligned}
& F(q) \mapsto F^{\left(\ell^{+}\right)}(q):=F(q)-\beta_{\ell} F\left(q^{\ell}\right) \in M_{k}\left(\Gamma_{0}\left(N^{\prime} \ell\right), \varepsilon_{F}\right), \\
& F(q) \mapsto F^{\left(\ell^{-}\right)}(q):=F(q)-\alpha_{\ell} F\left(q^{\ell}\right) \in M_{k}\left(\Gamma_{0}\left(N^{\prime} \ell\right), \varepsilon_{F}\right), \\
& F(q) \mapsto F^{\left(\ell^{0}\right)}(q):=F(q)-a_{\ell} F\left(q^{\ell}\right)+\varepsilon_{F}(\ell) \ell^{k-1} F\left(q^{\ell^{2}}\right) \in M_{k}\left(\Gamma_{0}\left(N^{\prime} \ell^{2}\right), \varepsilon_{F}\right) .
\end{aligned}
$$

Note that, for $\ell_{1} \neq \ell_{2}$, the stabilization operators $F \mapsto F^{\left(\ell_{1}^{\epsilon_{1}}\right)}$ and $F \mapsto F^{\left(\ell_{2}^{\epsilon_{2}}\right)}$ commute for any $\epsilon_{1}, \epsilon_{2} \in\{+,-, 0\}$. Then we define, for integers $S=\prod_{i} \ell_{i}^{e_{i}}$, $\epsilon, \epsilon_{1}, \epsilon_{2} \in\{+,-, 0\}$,

$$
F^{\left(S^{\epsilon}\right)}:=F^{\prod_{i}\left(\ell_{i}^{\epsilon}\right)}, F^{\left(S_{1}^{\epsilon 1} S_{2}^{\epsilon_{2}}\right)}:=F^{\left(S_{1}^{\epsilon 1}\right),\left(S_{2}^{\epsilon 2}\right)} .
$$

These operators clearly extend to $p$-adic modular forms. On the $p$-adic modular forms $\theta^{j} E_{k}^{\psi_{1}, \psi_{2}}$ from Section 4.1, we explicitly have

$$
\begin{aligned}
\theta^{j} E_{k}^{\psi_{1}, \psi_{2},\left(\ell^{+}\right)}(q) & :=\delta_{\psi_{1}=1, j=0} L\left(\psi_{1}, 1-k\right)\left(1-\psi_{2}(\ell) \ell^{k-1}\right)+2 \sum_{n=1}^{\infty} n^{j} \sigma_{k-1}^{\left.\psi_{1}, \psi_{2}, \ell^{+}\right)}(n) q^{n} \\
& =\theta^{j} E_{k}^{\psi_{1}, \psi_{2}}(q)-\psi_{2}(\ell) \ell^{k-1+j} \theta^{j} E_{k}^{\psi_{1}, \psi_{2}}\left(q^{\ell}\right),
\end{aligned}
$$

$\theta^{j} E_{k}^{\psi_{1}, \psi_{2},\left(\ell^{-}\right)}(q):=\delta_{\psi_{1}=1, j=0} L\left(\psi_{1}, 1-k\right)\left(1-\psi_{2}(\ell)\right)+2 \sum_{n=1}^{\infty} n^{j} \sigma_{k-1}^{\psi_{1}, \psi_{2},\left(\ell^{-}\right)}(n) q^{n}$ $=\theta^{j} E_{k}^{\psi_{1}, \psi_{2}}(q)-\psi_{1}(\ell) \ell^{j} \theta^{j} E_{k}^{\psi_{1}, \psi_{2}}\left(q^{\ell}\right)$,

$\theta^{j} E_{k}^{\psi_{1}, \psi_{2},\left(\ell^{0}\right)}(q):=\delta_{\psi_{1}=1, j=0} L\left(\psi_{1}, 1-k\right)\left(1-\psi_{1}(\ell)-\psi_{2}(\ell) \ell^{k-1}+\psi_{2}\left(\ell^{2}\right) \ell^{k-1}\right)$

$$
\begin{gathered}
+2 \sum_{n=1}^{\infty} n^{j} \sigma_{k-1}^{\psi_{1}, \psi_{2},\left(\ell^{0}\right)}(n) q^{n} \\
=\theta^{j} E_{k}^{\psi_{1}, \psi_{2}}(q)-\ell^{j}\left(\psi_{1}(\ell)+\psi_{2}(\ell) \ell^{k-1}\right) \theta^{j} E_{k}^{\psi_{1}, \psi_{2}}\left(q^{\ell}\right) \\
+\left(\psi_{1} \psi_{2}\right)(\ell) \ell^{k-1+2 j} \theta^{j} E\left(q^{\ell^{2}}\right),
\end{gathered}
$$


where $\delta_{\psi=1, j=0}$ equals 1 if $\psi$ is trivial and $j=0$, and 0 otherwise, and

$$
\begin{aligned}
\sigma_{k-1}^{\psi_{1}, \psi_{2},\left(\ell^{+}\right)}(n) & :=\sum_{\substack{0<d \mid n \\
(d, \ell)=1}} \psi_{1}(n / d) \psi_{2}(d) d^{k-1}, \\
\sigma_{k-1}^{\psi_{1}, \psi_{2},\left(\ell^{-}\right)}(n) & :=\sum_{\substack{0<d \mid n \\
(n / d, \ell)=1}} \psi_{1}(n / d) \psi_{2}(d) d^{k-1}, \\
\sigma_{k-1}^{\psi_{1}, \psi_{2},\left(\ell^{0}\right)}(n): & : \sum_{\substack{0<d \mid n \\
(n, \ell)=1}} \psi_{1}(n / d) \psi_{2}(d) d^{k-1} .
\end{aligned}
$$

Let $N^{\prime}$ be as in Section 4.1, let $N^{\prime \prime}=N_{+}^{\prime \prime} N_{-}^{\prime \prime} N_{0}^{\prime \prime}$ be prime to $N^{\prime}$, and suppose additionally that $K$ satisfies the Heegner hypothesis with respect to $N^{\prime \prime}$ so that every prime dividing $N:=N^{\prime \prime} N^{\prime}$ is split in $K$. Let $\mathfrak{N}=\mathfrak{N}^{\prime \prime} \mathfrak{N}^{\prime}$, where $\mathfrak{N}^{\prime}$ is as in Section 4.1 and $\mathfrak{N}^{\prime \prime}$ is some choice of integral ideal such that $\mathcal{O}_{K} / \mathfrak{N}^{\prime \prime}=\mathbb{Z} / N^{\prime \prime}$. Write $\mathfrak{N}$ as a product of (distinct) primes $\prod_{\ell \mid N} v$, where $v \mid \ell$; in other words, for each $\ell \mid N$, we can write

$$
v=\mathbb{Z} \frac{b_{\ell}+\sqrt{D_{K}}}{2}+\mathbb{Z} \ell
$$

for some $b_{\ell} \in \mathbb{Z}$ such that $b_{\ell}^{2} \equiv D_{K}(\bmod 4 \ell)$.

For any integral primitive ideal $\mathfrak{a}$ of $\mathcal{O}_{K}$ coprime to $\mathfrak{N}$, recall the associated point

$$
\tau_{\mathfrak{a} \mathfrak{N}}=\frac{b_{a N}+\sqrt{D_{K}}}{2 a N} \in \mathcal{H}^{+}
$$

such that $(\overline{\mathfrak{a} \mathfrak{N}})^{-1}=\mathbb{Z} \tau_{\mathfrak{a} \mathfrak{N}}+\mathbb{Z}$. Note that, for $v \mid \mathfrak{N}^{\prime \prime}$, where $v \mid \ell$, we have

$$
\bar{v}(\overline{\mathfrak{a} \mathfrak{N}})^{-1}=\left(\overline{\mathfrak{a} \prod_{v^{\prime} \neq v} v^{\prime}}\right)^{-1}=\mathbb{Z} \ell \frac{b_{a N}+\sqrt{D_{K}}}{2 a N}+\mathbb{Z},
$$

and hence

$$
\bar{v}^{-1} \star\left(\overline{\mathfrak{a} \mathfrak{N}} \star \mathbb{C} / \mathcal{O}_{K}\right)=\mathbb{C} /\left(\bar{v}(\overline{\mathfrak{a} \mathfrak{N}})^{-1}\right)=\mathbb{C} /\left(\mathbb{Z} \ell \tau_{\mathfrak{a} \mathfrak{N}}+\mathbb{Z}\right) .
$$

In terms of the action of $\square^{\mathfrak{N}}$ on CM triples $\left(A^{\prime}, t^{\prime}, \omega^{\prime}\right)$, for any $F \in M_{k}\left(\Gamma_{0}(N), \varepsilon_{F}\right)$, we have

$$
\begin{aligned}
F\left(\ell \tau_{\mathfrak{a} \mathfrak{N}}\right) & =F\left(\bar{v}^{-1} \star\left(\mathbb{C} /\left(\mathbb{Z} \tau_{\mathfrak{a} \mathfrak{N}}+\mathbb{Z}\right), t, 2 \pi i d z\right)\right)=F\left(\bar{v}^{-1} \overline{\mathfrak{a} \mathfrak{N}} \star\left(\mathbb{C} / \mathcal{O}_{K}, t, 2 \pi i d z\right)\right), \\
F\left(\ell^{2} \tau_{\mathfrak{a} \mathfrak{N}}\right) & =F\left(\bar{v}^{-2} \star\left(\mathbb{C} /\left(\mathbb{Z} \tau_{\mathfrak{a} \mathfrak{N}}+\mathbb{Z}\right), t, 2 \pi i d z\right)\right)=F\left(\bar{v}^{-2} \overline{\mathfrak{a} \mathfrak{N}} \star\left(\mathbb{C} / \mathcal{O}_{K}, t, 2 \pi i d z\right)\right) .
\end{aligned}
$$

Thus, for any normalized eigenform $F$, viewed as a $p$-adic modular form, recalling our notation $A_{0}=\mathbb{C} / \mathcal{O}_{K}, t_{0} \in A_{0}[\mathfrak{N}]$ from Section 3.4, and with $\omega_{\text {can }}$ as our 
"canonical" differential on $\hat{\mathcal{A}}_{0}$ under our fixed isomorphism $i: \hat{\mathcal{A}}_{0} \stackrel{\sim}{\longrightarrow} \hat{\mathbb{G}}_{m}$ (see Section 3.4), we have for $\ell \nmid N^{\prime}$

$\theta^{j} F^{\left(\ell^{+}\right)}\left(\overline{\mathfrak{a} \mathfrak{N}} \star\left(A_{0}, t_{0}, \omega_{\text {can }}\right)\right)$

$$
=\theta^{j} F\left(\overline{\mathfrak{a} \mathfrak{N}} \star\left(A_{0}, t_{0}, \omega_{\text {can }}\right)\right)-\beta_{\ell} \ell^{j} \theta^{j} F\left(\bar{v}^{-1} \overline{\mathfrak{a} \mathfrak{N}} \star\left(A_{0}, t_{0}, \omega_{\text {can }}\right)\right),
$$

$\theta^{j} F^{\left(\ell^{-}\right)}\left(\overline{\mathfrak{a} \mathfrak{N}} \star\left(A_{0}, t_{0}, \omega_{\text {can }}\right)\right)$

$$
=\theta^{j} F\left(\overline{\mathfrak{a} \mathfrak{N}} \star\left(A_{0}, t_{0}, \omega_{\text {can }}\right)\right)-\alpha_{\ell} \ell^{j} \theta^{j} F\left(\bar{v}^{-1} \overline{\mathfrak{a} \mathfrak{N}} \star\left(A_{0}, t_{0}, \omega_{\text {can }}\right)\right),
$$

$\theta^{j} F^{\left(\ell^{0}\right)}\left(\overline{\mathfrak{a} \mathfrak{N}} \star\left(A_{0}, t_{0}, \omega_{\text {can }}\right)\right)$

$$
\begin{gathered}
=\theta^{j} F\left(\overline{\mathfrak{a} \mathfrak{N}} \star\left(A_{0}, t_{0}, \omega_{\text {can }}\right)\right)-\ell^{j} a_{\ell} \theta^{j} F\left(\bar{v}^{-1} \overline{\mathfrak{a} \mathfrak{N}} \star\left(A_{0}, t_{0}, \omega_{\text {can }}\right)\right) \\
+\varepsilon_{F}(\ell) \ell^{k-1+2 j} \theta^{j} F\left(\bar{v}^{-2} \overline{\mathfrak{a} \mathfrak{N}} \star\left(A_{0}, t_{0}, \omega_{\text {can }}\right)\right) .
\end{gathered}
$$

Choosing some set of representatives $\mathfrak{a}$ prime to $\mathfrak{N}$, we thus have

$\sum_{[\mathfrak{a}] \in \operatorname{Cl}\left(\mathcal{O}_{K}\right)}\left(\chi_{j}\right)^{-1}(\mathfrak{a}) \theta^{j} F^{\left(\ell^{+}\right)}\left(\mathfrak{a} \star\left(A_{0}, t_{0}, \omega_{\text {can }}\right)\right)$

$=\sum_{[\mathfrak{a}] \in \operatorname{Cl}\left(\mathcal{O}_{K}\right)}\left(\chi_{j}\right)^{-1}(\mathfrak{a}) \theta^{j} F\left(\mathfrak{a} \star\left(A_{0}, t_{0}, \omega_{\text {can }}\right)\right)$

$$
-\beta_{\ell} \ell^{j} \sum_{\left[\mathrm{a} \in \mathrm{Cl}\left(\mathcal{O}_{K}\right)\right.}\left(\chi_{j}\right)^{-1}(\mathfrak{a}) \theta^{j} F\left(\bar{v}^{-1} \mathfrak{a} \star\left(A_{0}, t_{0}, \omega_{\text {can }}\right)\right)
$$

$[\mathfrak{a}] \in \mathrm{Cl}\left(\mathcal{O}_{K}\right)$

$=\left(1-\beta_{\ell}\left(\chi_{j}\right)^{-1}(\bar{v}) \ell^{j}\right) \sum_{[\mathfrak{a}] \in \operatorname{Cl}\left(\mathcal{O}_{K}\right)}\left(\chi_{j}\right)^{-1}(\mathfrak{a}) \theta^{j} F\left(\mathfrak{a} \star\left(A_{0}, t_{0}, \omega_{\text {can }}\right)\right)$,

$\sum_{\left[\mathfrak{a} \in \mathbb{C}\left(\mathcal{O}_{K}\right)\right.}\left(\chi_{j}\right)^{-1}(\mathfrak{a}) \theta^{j} F^{\left(\ell^{-}\right)}\left(\mathfrak{a} \star\left(A_{0}, t_{0}, \omega_{\text {can }}\right)\right)$

$[\mathfrak{a}] \in \mathrm{Cl}\left(\mathcal{O}_{K}\right)$

$=\sum_{[\mathfrak{a}] \in \operatorname{Cl}\left(\mathcal{O}_{K}\right)}\left(\chi_{j}\right)^{-1}(\mathfrak{a}) \theta^{j} F\left(\mathfrak{a} \star\left(A_{0}, t_{0}, \omega_{\text {can }}\right)\right)$

$$
-\alpha_{\ell} \ell^{j} \sum_{[\mathfrak{a}] \in \operatorname{Cl}\left(\mathcal{O}_{K}\right)}\left(\chi_{j}\right)^{-1}(\mathfrak{a}) \theta^{j} F\left(\bar{v}^{-1} \mathfrak{a} \star\left(A_{0}, t_{0}, \omega_{\text {can }}\right)\right)
$$

$=\left(1-\alpha_{\ell}\left(\chi_{j}\right)^{-1}(\bar{v}) \ell^{j}\right) \sum\left(\chi_{j}\right)^{-1}(\mathfrak{a}) \theta^{j} F\left(\mathfrak{a} \star\left(A_{0}, t_{0}, \omega_{\text {can }}\right)\right)$, $[\mathfrak{a}] \in \operatorname{Cl}\left(\mathcal{O}_{K}\right)$

$[\mathfrak{a}] \in \operatorname{Cl}\left(\mathcal{O}_{K}\right)$

$$
\begin{aligned}
=\sum_{[\mathfrak{a}] \in \operatorname{Cl}\left(\mathcal{O}_{K}\right)}\left(\chi_{j}\right)^{-1}(\mathfrak{a}) \theta^{j} F\left(\mathfrak{a} \star\left(A_{0}, t_{0}, \omega_{\text {can }}\right)\right) \\
\quad-\ell^{j} a_{\ell} \sum_{[\mathfrak{a}] \in \operatorname{Cl}\left(\mathcal{O}_{K}\right)}\left(\chi_{j}\right)^{-1}(\mathfrak{a}) \theta^{j} F\left(\bar{v}^{-1} \mathfrak{a} \star\left(A_{0}, t_{0}, \omega_{\text {can }}\right)\right) \\
\quad+\varepsilon_{F}(\ell) \ell^{k-1+2 j} \sum_{[\mathfrak{a}] \in \operatorname{Cl}\left(\mathcal{O}_{K}\right)}\left(\chi_{j}\right)^{-1}(\mathfrak{a}) \theta^{j} F\left(\bar{v}^{-2} \mathfrak{a} \star\left(A_{0}, t_{0}, \omega_{\text {can }}\right)\right)
\end{aligned}
$$




$$
\begin{aligned}
=(1 & -a_{\ell}\left(\chi_{j}\right)^{-1}(\bar{v}) \ell^{j} \\
& \left.+\varepsilon_{F}(\ell)\left(\chi_{j}\right)^{-2}(\bar{v}) \ell^{k-1+2 j}\right) \sum_{[\mathfrak{a}] \in \operatorname{Cl}\left(\mathcal{O}_{K}\right)}\left(\chi_{j}\right)^{-1}(\mathfrak{a}) \theta^{j} F\left(\mathfrak{a} \star\left(A_{0}, t_{0}, \omega_{\text {can }}\right)\right) .
\end{aligned}
$$

Thus, rewriting the definitions of $E_{k}^{\psi_{1}, \psi_{2},\left(\left(N_{+}^{\prime \prime}\right)^{+}\left(N_{-}^{\prime \prime}\right)^{-}\left(N_{0}^{\prime \prime}\right)^{0}\right)}$ in terms of triples, and using the above general identities for $p$-adic modular forms and induction, we have

$$
\begin{aligned}
\sum_{[\mathfrak{a}] \in \operatorname{Cl}\left(\mathcal{O}_{K}\right)}\left(\chi_{j}\right)^{-1}(\mathfrak{a}) \theta^{j} E_{k}^{\psi_{1}, \psi_{2},\left(\left(N_{+}^{\prime \prime}\right)^{+}\left(N_{-}^{\prime \prime}\right)^{-}\left(N_{0}^{\prime \prime}\right)^{0}\right)}\left(\mathfrak{a} \star\left(A_{0}, t_{0}, \omega_{\text {can }}\right)\right) \\
=\Xi_{\chi}\left(\psi_{1}, \psi_{2}, N_{+}, N_{-}, N_{0}\right) \sum_{[\mathfrak{a}] \in \operatorname{Cl}\left(\mathcal{O}_{K}\right)}\left(\chi_{j}\right)^{-1}(\mathfrak{a}) \theta^{j} E_{k}^{\psi_{1}, \psi_{2}}\left(\mathfrak{a} \star\left(A_{0}, t_{0}, \omega_{\text {can }}\right)\right),
\end{aligned}
$$

where $N_{+}=N_{+}^{\prime \prime} \cdot t /(u, t), N_{-}=N_{-}^{\prime \prime} \cdot u /(u, t), N_{0}=N_{0}^{\prime \prime} \cdot(u, t)^{2}$, and

$$
\begin{aligned}
& \Xi_{\chi}\left(\psi_{1}, \psi_{2}, N_{+}, N_{-}, N_{0}\right) \\
& =\prod_{\ell \mid N_{+}}\left(1-\left(\psi_{2 / K} \chi^{-1}\right)(\bar{v}) \ell^{k-1}\right) \prod_{\ell \mid N_{-}}\left(1-\left(\psi_{1 / K} \chi^{-1}\right)(\bar{v})\right) \\
& \quad \times \prod_{\ell \mid N_{0}}\left(1-\left(\psi_{2 / K} \chi^{-1}\right)(\bar{v}) \ell^{k-1}\right)\left(1-\left(\psi_{1 / K} \chi^{-1}\right)(\bar{v})\right) .
\end{aligned}
$$

Henceforth, let $E_{k}^{\left(\psi_{1}, \psi_{2}, N_{+}, N_{-}, N_{0}\right)}:=E_{k}^{\psi_{1}, \psi_{2},\left(\left(N_{+}^{\prime \prime}\right)^{+}\left(N_{-}^{\prime \prime}\right)^{-}\left(N_{0}^{\prime \prime}\right)^{0}\right)}$. Now, using the above calculation, Proposition 36, the fact that

$$
\omega_{\mathrm{can}}=\frac{\Omega_{\infty}}{\Omega_{p}} \cdot 2 \pi i d z
$$

(where $\Omega_{\infty}$ and $\Omega_{p}$ are the complex and $p$-adic periods defined in Section 3.4) and part (3) of Theorem 21, we have:

Proposition 37. Let $k \geq 2$ be an integer. Suppose $\chi$ is of infinity type $(k+j,-j)$ and $\chi_{-k / 2}$ has trivial central character and is of finite type $\left(\mathfrak{N}, \psi_{1} \psi_{2}\right)$. Suppose also that $\psi_{1}$ and $\psi_{2}$ are Dirichlet characters over $\mathbb{Q}$ with conductors $u$ and $t$, respectively, such that $u t=N$ and $\left(\psi_{1} \psi_{2}\right)(-1)=(-1)^{k}$. Then

$$
\begin{aligned}
& \sum_{[\mathfrak{a}] \in \operatorname{Cl}\left(\mathcal{O}_{K}\right)}\left(\chi_{j}\right)^{-1}(\mathfrak{a}) \theta^{j} E_{k}^{\left(\psi_{1}, \psi_{2}, N_{+}, N_{-}, N_{0}\right)}\left(\mathfrak{a} \star\left(A_{0}, t_{0}, \omega_{\text {can }}\right)\right) \\
& =\left(\frac{\Omega_{p}}{\Omega_{\infty}}\right)^{k+2 j} \frac{t^{k} \Gamma(k+j) \psi_{1}^{-1}\left(-\sqrt{D_{K}}\right) \chi^{-1}(\overline{\mathfrak{t}})}{(2 \pi i)^{k+j} \mathfrak{g}\left(\psi_{2}^{-1}\right){\sqrt{D_{K}}}^{j}} \\
& \quad \times \Xi_{\chi}\left(\psi_{1}, \psi_{2}, N_{+}, N_{-}, N_{0}\right) L\left(\psi_{1 / K} \chi^{-1}, 0\right),
\end{aligned}
$$


where

$$
\begin{aligned}
& \Xi_{\chi}\left(\psi_{1}, \psi_{2}, N_{+}, N_{-}, N_{0}\right) \\
& =\prod_{\ell \mid N_{+}}\left(1-\left(\psi_{2 / K} \chi^{-1}\right)(\bar{v}) \ell^{k-1}\right) \prod_{\ell \mid N_{-}}\left(1-\left(\psi_{1 / K} \chi^{-1}\right)(\bar{v})\right) \\
& \prod_{\ell \mid N_{0}}\left(1-\left(\psi_{2 / K} \chi^{-1}\right)(\bar{v}) \ell^{k-1}\right)\left(1-\left(\psi_{1 / K} \chi^{-1}\right)(\bar{v})\right) .
\end{aligned}
$$

Finally, since $p \nmid N$, note that the " $p$-depletion" operator b of Section 3.2 coincides with the $\left(\left(p^{2}\right)^{0}\right)$-stabilization operator on our family of Eisenstein series, i.e.,

$$
\left(\theta^{j} E_{k}^{\left(\psi_{1}, \psi_{2}, N_{+}, N_{-}, N_{0}\right)}\right)^{\mathrm{b}}=\theta^{j} E_{k}^{\left(\psi_{1}, \psi_{2}, N_{+}, N_{-}, p^{2} N_{0}\right)} .
$$

(Note that all the above stabilization operators on $p$-adic modular forms commute with $\theta$, so the above notation is unambiguous.)

\subsection{Proof of main theorem.}

Proof of Theorem 3. Suppose, as in our assumptions, that $f \in S_{k}\left(\Gamma_{0}(N), \varepsilon_{f}\right)$ has partial Eisenstein descent of type $\left(\psi_{1}, \psi_{2}, N_{+}, N_{-}, N_{0}\right)$ over $M \bmod \mathfrak{m}$, where $M$ is a $p$-adic field containing $E_{f}$. Recall the field $F^{\prime}$ defined in Section 3.4, with $\mathfrak{p}^{\prime}$ being the prime ideal of $\mathcal{O}_{F^{\prime}}$ above $p$ determined by our embedding $i_{p}: \overline{\mathbb{Q}}_{\hookrightarrow} \overline{\mathbb{Q}}_{p}$. Thus,

$$
\theta^{j} f(q) \equiv \theta^{j} E_{k}^{\left(\psi_{1}, \psi_{2}, N_{+}, N_{-}, N_{0}\right)}(q)\left(\bmod \mathfrak{m} \mathcal{O}_{F_{\mathfrak{p}^{\prime}} M}\right)
$$

viewed as $p$-adic modular forms for all $j \geq 1$. Moreover,

$$
\theta^{j} f^{b}(q) \equiv \theta^{j} E_{k}^{\left(\psi_{1}, \psi_{2}, N_{+}, N_{-}, p^{2} N_{0}\right)}(q)\left(\bmod \mathfrak{m} \mathcal{O}_{F_{\mathfrak{p}^{\prime}}^{\prime} M}\right)
$$

Henceforth, let $E_{k, \psi_{1}, \psi_{2}}^{(N)}=E_{k}^{\left(\psi_{1}, \psi_{2}, N_{+}, N_{-}, N_{0}\right)}$ and $E_{k, \psi_{1}, \psi_{2}}^{(p N)}=E_{k}^{\left(\psi_{1}, \psi_{2}, N_{+}, N_{-}, p^{2} N_{0}\right)}$, and recall our notation $A_{0}=\mathbb{C} / \mathcal{O}_{K}, t_{0} \in A_{0}[\mathfrak{N}]$ (see Section 3.4). Since $p$ being split in $K$ implies that the curves $i_{p}\left(\mathfrak{a} \star A_{0}\right)$ are ordinary (viewed as curves over $\mathbb{C}_{p}$ ), by the congruence above and the $q$-expansion principle [Gouvêa 1988, Sections I.3.2 and I.3.5] we have

$$
\begin{aligned}
& \sum_{\mathfrak{a} \in \operatorname{Cl}\left(\mathcal{O}_{K}\right)}\left(\chi_{j}\right)^{-1}(\mathfrak{a})\left(\theta^{j} f\right)^{\mathfrak{b}}\left(\mathfrak{a} \star\left(A_{0}, t_{0}, \omega_{\text {can }}\right)\right) \\
& \quad \equiv \sum_{\mathfrak{a} \in \operatorname{Cl}\left(\mathcal{O}_{K}\right)}\left(\chi_{j}\right)^{-1}(\mathfrak{a})\left(\theta^{j} E_{k, \psi_{1}, \psi_{2}}^{(p N)}\right)\left(\mathfrak{a} \star\left(A_{0}, t_{0}, \omega_{\text {can }}\right)\right)\left(\bmod \mathfrak{m} \mathcal{O}_{F_{\mathfrak{p}^{\prime}}, M}\right) .
\end{aligned}
$$


By assumption, $\varepsilon_{f}$ equals $\psi_{1} \psi_{2}$ and $\chi$ is of finite type $\left(\mathfrak{N}, \varepsilon_{f}\right)=\left(\mathfrak{N}, \psi_{1} \psi_{2}\right)$. Now by Proposition 37, we have

$$
\begin{aligned}
& \sum_{\mathfrak{a} \in \mathrm{Cl}\left(\mathcal{O}_{K}\right)}\left(\chi_{j}\right)^{-1}(\mathfrak{a})\left(\theta^{j}\right.\left.E_{k, \psi_{1}, \psi_{2}}^{(p N)}\right)\left(\mathfrak{a} \star\left(A_{0}, t_{0}, \omega_{\text {can }}\right)\right) \\
&=\left(\frac{\Omega_{p}}{\Omega_{\infty}}\right)^{k+2 j} \frac{t^{k} \Gamma(k+j) \psi_{1}^{-1}\left(-\sqrt{D_{K}}\right) \chi^{-1}(\overline{\mathfrak{t}})}{(2 \pi i)^{k+j} \mathfrak{g}\left(\psi_{2}^{-1}\right){\sqrt{D_{K}}}^{j}} \\
& \times \Xi_{\chi}\left(\psi_{1}, \psi_{2}, N_{+}, N_{-}, p^{2} N_{0}\right) L\left(\psi_{1 / K} \chi^{-1}, 0\right),
\end{aligned}
$$

where $\Xi_{\chi}\left(\psi_{1}, \psi_{2}, N_{+}, N_{-}, p^{2} N_{0}\right)$ is defined as in Proposition 37.

Since $\chi$ is of finite type $\left(\mathfrak{N}, \varepsilon_{f}\right)$, it is unramified at $\mathfrak{p}$. Moreover, since $\mathfrak{p} \nmid u \mathcal{O}_{K}$, $\psi_{1 / K}$ is unramified at $\mathfrak{p}$. Hence, by [Katz 1978, 5.2.27 Lemma, 5.2.28], we have $\operatorname{Local}_{\mathfrak{p}}\left(\psi_{1 / K} \chi^{-1}\right)=1$ and $\operatorname{Local}_{\mathfrak{p}}\left(\psi_{1 / K} \chi^{-1} \mathbb{N}_{K}^{1-k}\right)=1$.

Now using Theorems 24 and 27 with $\mathfrak{C}=\mathfrak{f}\left(\psi_{1 / K} \chi^{-1}\right)=\operatorname{lcm}\left(u \mathcal{O}_{K}, \mathfrak{t}\right)$, where $\mathfrak{F}=\operatorname{lcm}(\mathfrak{u}, \mathfrak{t}), \mathfrak{F}_{c}=\overline{\mathfrak{u}}$ and $\mathfrak{I}=1$, we can write $\mathcal{L}_{p}(f, \chi)$

$$
\begin{aligned}
& =\left(\sum_{\mathfrak{a} \in \operatorname{Cl}\left(\mathcal{O}_{K}\right)}\left(\chi_{j}\right)^{-1}(\mathfrak{a})\left(\theta^{j} f\right)^{\mathfrak{b}}\left(\mathfrak{a} \star\left(A_{0}, t_{0}, \omega_{\text {can }}\right)\right)\right)^{2} \\
& \equiv\left(\sum_{\mathfrak{a} \in \operatorname{Cl}\left(\mathcal{O}_{K}\right)}\left(\chi_{j}\right)^{-1}(\mathfrak{a})\left(\theta^{j} E_{k, \psi_{1}, \psi_{2}}^{(p N)}\right)\left(\mathfrak{a} \star\left(A_{0}, t_{0}, \omega_{\mathrm{can}}\right)\right)\right)^{2} \\
& =\left(\left(\frac{\Omega_{p}}{\Omega_{\infty}}\right)^{k+2 j} \frac{t^{k} \Gamma(k+j) \psi_{1}^{-1}\left(-\sqrt{D_{K}}\right) \chi^{-1}(\overline{\mathfrak{t}})}{(2 \pi i)^{k+j} \mathfrak{g}\left(\psi_{2}^{-1}\right){\sqrt{D_{K}}}^{j}}\right. \\
& \left.\quad \times \Xi_{\chi}\left(\psi_{1}, \psi_{2}, N_{+}, N_{-}, p^{2} N_{0}\right) L\left(\psi_{1 / K} \chi^{-1}, 0\right)\right)^{2} \\
& =\psi_{1}^{-1}\left(D_{K}\right)\left(\frac{t^{k} \chi^{-1}(\overline{\mathfrak{t}})}{4 \mathfrak{g}\left(\psi_{2}^{-1}\right)(2 \pi i)^{k+2 j}} \Xi_{\chi}\left(\psi_{1}, \psi_{2}, N_{+}, N_{-}, N_{0}\right) L_{p}\left(\psi_{1 / K} \chi^{-1}, 0\right)\right)^{2} \\
& \quad\left(\bmod \mathfrak{m} \mathcal{O}_{F_{\mathfrak{p}^{\prime}} M}\right) .
\end{aligned}
$$

The above congruence now extends by $p$-adic continuity from $\Sigma_{\mathrm{cc}}^{(2)}(\mathfrak{N})$ to $\hat{\Sigma}_{\mathrm{cc}}(\mathfrak{N})$ (with respect to the topology described in Section 3.4). Thus the congruence holds on $\hat{\Sigma}_{\mathrm{cc}}(\mathfrak{N})$, and the theorem follows after setting $\Xi:=\Xi_{\chi}\left(\psi_{1}, \psi_{2}, N_{+}, N_{-}, N_{0}\right)$.

Proof of Theorem 7. In this more specialized situation, we have $\psi_{1}=\psi_{2}^{-1}=\psi$ and $u=t=|\mathfrak{f}(\psi)|$. Thus $\psi_{1} \psi_{2}$ equals 1 and $\chi$ is of finite type $(\mathfrak{N}, 1)$. Recall from Bertolini, Darmon, and Prasanna's $p$-adic Waldspurger formula (Theorem 25) that

$$
\mathcal{L}_{p}\left(f, \mathbb{N}_{K}^{-k / 2}\right)=\left(\frac{p^{k / 2}-a_{p}(f)+p^{k / 2-1}}{p^{k / 2} \Gamma\left(\frac{k}{2}\right)}\right)^{2}\left(\mathrm{AJ}_{F_{\mathfrak{p}^{\prime}}}\left(\Delta_{f}(K)\right)\left(\omega_{f} \wedge \omega_{A}^{k / 2-1} \eta_{A}^{k / 2-1}\right)\right)^{2} .
$$


The proof proceeds essentially by plugging $\chi=\mathbb{N}_{K}^{-k / 2}$ into Theorem 3 . We have, by Gross's factorization formula (Theorem 28) applied to $\left(\psi \omega^{-k / 2}\right)_{0}$ (see Definition 6),

$$
\begin{aligned}
& \frac{|\mathfrak{f}(\psi)|^{k / 2}}{\psi\left(-\sqrt{D_{K}}\right) \mathfrak{g}(\psi)} L_{p}\left(\psi_{/ K} \mathbb{N}_{K}^{k / 2}, 0\right) \\
& =\frac{\langle|\mathfrak{f}(\psi)|\rangle^{k / 2}}{\prod_{\ell \mid \mathfrak{f}(\psi)}\left(\psi^{-1} \omega^{k / 2}\right)_{0, \ell}\left(-\sqrt{D_{K}}\right) \omega_{\ell}^{-k / 2}\left(\ell^{\operatorname{ord}_{\ell}(\mathfrak{f}(\psi))}\right) \mathfrak{g}_{\ell}(\psi)} L_{p}\left(\left(\psi \omega^{-k / 2}\right)_{0 / K}, \frac{k}{2}\right) \\
& =\frac{\langle|\mathfrak{f}(\psi)|\rangle^{k / 2}}{\prod_{\ell \mid \mathfrak{f}(\psi)}\left(\psi^{-1} \omega^{k / 2}\right)_{0, \ell}\left(-\sqrt{D_{K}}\right) \mathfrak{g}_{\ell}\left(\left(\psi \omega^{-k / 2}\right)_{0}\right)} L_{p}\left(\left(\psi \omega^{-k / 2}\right)_{0 / K}, \frac{k}{2}\right) \\
& =L_{p}\left(\psi_{0}\left(\varepsilon_{K} \omega\right)^{1-k / 2}, \frac{k}{2}\right) L_{p}\left(\psi_{0}^{-1}\left(\varepsilon_{K} \omega\right)^{k / 2}, 1-\frac{k}{2}\right) .
\end{aligned}
$$

Using the interpolation property of the Kubota-Leopoldt $p$-adic $L$-function, we have

$$
\begin{aligned}
L_{p}\left(\psi_{0}^{-1}\left(\varepsilon_{K} \omega\right)^{k / 2}, 1-\frac{k}{2}\right) & =-\left(1-\left(\psi_{0}^{-1} \varepsilon_{K}^{k / 2}\right)(p) p^{k / 2-1}\right) \frac{2}{k} B_{\frac{k}{2}, \psi_{0}^{-1} \varepsilon_{K}^{k / 2}} \\
& =-\left(1-\psi_{0}^{-1}(p) p^{k / 2-1}\right) \frac{2}{k} B_{\frac{k}{2}, \psi_{0}^{-1} \varepsilon_{K}^{k / 2},}
\end{aligned}
$$

where the last equality follows since $p$ is split in $K$ and so $\varepsilon_{K}(p)=1$.

Suppose $\psi_{0}\left(\varepsilon_{K} \omega\right)^{1-k / 2} \neq 1$. In this case, since $p^{2} \nmid \mathfrak{f}\left(\psi_{0}\left(\varepsilon_{K} \omega\right)^{1-k / 2}\right) \mid p f$, we have by Corollary 5.13 of [Washington 1997] that

$$
\begin{aligned}
& L_{p}\left(\psi_{0}\left(\varepsilon_{K} \omega\right)^{1-k / 2}, \frac{k}{2}\right) \equiv L_{p}\left(\psi_{0}\left(\varepsilon_{K} \omega\right)^{1-k / 2}, 0\right) \\
& =-\left(1-\left(\psi_{0} \varepsilon_{K}\left(\varepsilon_{K} \omega\right)^{-k / 2}\right)(p)\right) B_{1, \psi_{0} \varepsilon_{K}\left(\varepsilon_{K} \omega\right)^{-k / 2}} \\
& =-B_{1, \psi_{0} \varepsilon_{K}\left(\varepsilon_{K} \omega\right)^{-k / 2}}(\bmod p) \text {, }
\end{aligned}
$$

where the last equality holds since $p \mid \mathfrak{f}\left(\psi_{0} \varepsilon_{K}\left(\varepsilon_{K} \omega\right)^{1-k / 2}\right)$. Note that this congruence also holds mod $\lambda$ for any prime $\lambda \mid p$.

Now suppose $\psi_{0}\left(\varepsilon_{K} \omega\right)^{1-k / 2}=1$ and $k=2$, i.e., $\psi_{0}=1$. From [Gross 1980, p. 90], the Katz $p$-adic $L$-function at $\psi_{0 / K}=1_{/ K}$ has the special value

$$
L_{p}\left(1_{/ K}, 0\right)=\frac{4}{\left|\mathcal{O}_{K}^{\times}\right|} \frac{p-1}{p} \log _{p} \bar{\alpha}=2 \frac{p-1}{p} \log _{p} \bar{\alpha},
$$

where $\bar{\alpha} \in \mathcal{O}_{K}$ such that $(\bar{\alpha})=\overline{\mathfrak{p}}^{h_{K}}$. (Recall that $\left|\mathcal{O}_{K}^{\times}\right|=2$ since we assume that $D_{K}<-4$.) The statement now follows directly from Theorem 3 .

\section{Concrete applications of the main theorem}

In this section, we apply Theorem 7 to computations with algebraic cycles, in certain instances verifying a weak form of the Beilinson-Bloch conjecture (as described 
in Section 2.2). In Section 5.5, we apply our results to the case of elliptic curves with reducible mod- $p$ Galois representation, in particular deriving criteria to show that Heegner points on certain quadratic twists are nontorsion. In Section 5.6, we use this criterion to show that for semistable curves with reducible mod-3 Galois representation, a positive proportion of real quadratic twists have rank 1 and a positive proportion of imaginary quadratic twists have rank 0 .

5.1. Construction of newforms with Eisenstein descent of type $(1,1,1,1,1)$. We now give a procedure for constructing newforms $f \in S_{k}\left(\mathrm{SL}_{2}(\mathbb{Z})\right)$ which have Eisenstein descent of type $(1,1,1,1,1)$.

Construction 38 (Eisenstein descent of type $(1,1,1,1,1)$ ). Fix an even integer $k \geq 4$ and a prime $p \mid B_{k}$. Recall the classical holomorphic Eisenstein series of weight $k$ :

$$
E_{k}(q)=\frac{\zeta(1-k)}{2}+\sum_{n=1}^{\infty} \sigma_{k-1}(n) q^{n}=-\frac{B_{k}}{2 k}+\sum_{n=1}^{\infty} \sigma_{k-1}(n) q^{n} .
$$

(See Remark 32.) The Eisenstein series $E_{4}$ and $E_{6}$ generate $M_{k}\left(\mathrm{SL}_{2}(\mathbb{Z})\right) / S_{k}\left(\mathrm{SL}_{2}(\mathbb{Z})\right)$ as an algebra, and therefore if we normalize $E_{4}$ and $E_{6}$ to $G_{4}=240 E_{4}$ and $G_{6}=-504 E_{6}$ to have constant term 1 , then we have a cuspform

$$
f_{k}:=E_{k}+\frac{B_{k}}{2 k} G_{4}^{a} G_{6}^{b} \in S_{k}\left(\mathrm{SL}_{2}(\mathbb{Z}), \mathbb{Q}\right)
$$

for some $a, b \in \mathbb{Z}_{\geq 0}$ with $k=4 a+6 b$.

Under our assumption $p \mid B_{k}$, we see immediately that $f_{k} \equiv E_{k}(\bmod p)$. However, there is no guarantee that $f_{k}$ is a newform, a problem we can remedy as follows. Consider the Hecke algebra $\mathbb{T} \subset \operatorname{End}_{\mathbb{Z}_{p}}\left(S_{k}\left(\mathrm{SL}_{2}(\mathbb{Z}), \mathbb{Z}_{p}\right)\right)$ generated by the classical Hecke operators $T_{\ell}$ at primes $\ell$. Since $\mathbb{T}$ is a finite torsion-free $\mathbb{Z}_{p}$-algebra, it is flat. Minimal primes $\mathfrak{p}$ of $\mathbb{T}$ correspond to $\operatorname{Gal}\left(\overline{\mathbb{Q}}_{p} / \mathbb{Q}_{p}\right)$-conjugacy classes of weight- $k$ eigenforms in $S_{k}\left(\mathrm{SL}_{2}(\mathbb{Z})\right)$, which also correspond to $\mathbb{Z}_{p}$-algebra homomorphisms $\phi: \mathbb{T} \rightarrow \overline{\mathbb{Q}}_{p}($ with $\operatorname{ker}(\phi)=\mathfrak{p})$, which in turn correspond to eigenforms $f_{\phi}$ such that $T_{\ell} f_{\phi}=\phi\left(T_{\ell}\right) f_{\phi}$.

Now write $f_{k}(q)=\sum_{n=1}^{\infty} a_{n} q^{n}$ so that $a_{n} \equiv \sigma_{k-1}(n)(\bmod p)$, and define a homomorphism $\varphi: \mathbb{T} \rightarrow \mathbb{F}_{p}$ given by $\varphi\left(T_{\ell}\right)=a_{\ell} \equiv 1+\ell^{k-1}(\bmod p)$. Since $\mathbb{F}_{p}$ is a field, $\mathfrak{m}:=\operatorname{ker}(\varphi)$ is maximal, and there exists a minimal prime $\mathfrak{p} \subset \mathfrak{m}$ above $p$ since $\mathbb{T}$ is flat over $\mathbb{Z}_{p}$. Then the minimal prime $\mathfrak{p}$ corresponds to a map $\phi: \mathbb{T} \rightarrow \overline{\mathbb{Q}}_{p}$ with $\operatorname{ker}(\phi)=\mathfrak{p}$ that satisfies $\phi \equiv \varphi(\bmod p)$. Let $F=\operatorname{Frac}(\phi(\mathbb{\mathbb { T }}))$ denote the field of fractions of $\phi(\mathbb{T})$, and note that $\phi(\mathbb{T}) \subset \mathcal{O}_{F}$; since $\mathbb{T}$ is finite over $\mathbb{Z}_{p}, F$ is finite over $\mathbb{Q}_{p}$. Now let $\lambda$ denote the maximal ideal of $\mathcal{O}_{F}$. Since $T_{\ell} \equiv 1+\ell^{k-1}(\bmod \mathfrak{m})$ (because $\varphi: \mathbb{T} / \mathfrak{m} \stackrel{\sim}{\longrightarrow} \mathbb{F}_{p}$ ), we have $\phi\left(T_{\ell}\right) \equiv 1+\ell^{k-1}(\bmod \phi(\mathfrak{m}))$. Hence since 
$\lambda \mid \phi(\mathfrak{m})$, the modular form $f_{\phi}$ corresponding to $\phi$ satisfies

$$
f_{\phi}(q) \equiv \sum_{n=1}^{\infty} \sigma_{k-1}(n) q^{n}(\bmod \lambda)
$$

and is our desired newform.

Remark 39. The last paragraph of Construction 38 is commonly known as the "Deligne-Serre lifting lemma" (see [Deligne and Serre 1974, Lemme 6.11]).

5.2. Construction of newforms with Eisenstein descent of type $\left(1,1, N_{+}, N_{-}, N_{0}\right)$. Construction 40 (Eisenstein descent of type $\left(1,1, N_{+}, N_{-}, N_{0}\right)$ ). We can easily use the normalized newform with Eisenstein descent of type $(1,1,1,1,1)$ obtained from Construction 38 to produce a newform of descent of type $\left(1,1, N_{+}, N_{-}, N_{0}\right)$. We apply certain stabilization operators, as in Section 4.2. Suppose we are given a normalized newform $f \in S_{k}\left(\Gamma_{1}\left(N^{\prime}\right)\right)$ with Eisenstein descent of type $\left(1,1, N_{+}^{\prime}, N_{-}^{\prime}, N_{0}^{\prime}\right)$ $\bmod \lambda$. Applying the $\left(N_{+}^{+} N_{-}^{-} N_{0}^{0}\right)$-stabilization operator, the resulting newform $f^{\left(N_{+}^{+} N_{-}^{-} N_{0}^{0}\right)} \in S_{k}\left(\Gamma_{1}\left(N^{\prime}\right) \cap \Gamma_{0}(N)\right)$ is a normalized newform which has Eisenstein descent of type $\left(1,1, N_{+}^{\prime} N_{+}, N_{-}^{\prime} N_{-}, N_{0}^{\prime} N_{0}\right) \bmod \lambda$.

Applying the above $\left(N_{+}^{+} N_{-}^{-} N_{0}^{0}\right)$-stabilization operator to $f_{\phi} \in S_{k}\left(\mathrm{SL}_{2}(\mathbb{Z})\right)$ from Construction 38, we have

$$
f_{\phi}^{\left(N_{+}^{+} N_{-}^{-} N_{0}^{0}\right)}(q) \equiv \sum_{n=1}^{\infty} \sigma_{k-1}^{(N)}(n) q^{n}(\bmod \lambda)
$$

where $\sigma_{k-1}^{(N)}$ is defined as in Remark 32. In other words, $f_{\phi}^{\left(N_{+}^{+} N_{-}^{-} N_{0}^{0}\right)}$ has Eisenstein descent of type $\left(1,1, N_{+}, N_{-}, N_{0}\right) \bmod \lambda$.

Remark 41. We could similarly produce examples of newforms with Eisenstein descent of type $\left(\psi_{1}, \psi_{2}, N_{+}, N_{-}, N_{0}\right)$ by starting out with the Eisenstein series $E_{k}^{\psi_{1}, \psi_{2}}$ in Construction 38 and applying appropriate stabilization operators as in Construction 40 .

5.3. Application to algebraic cycles. We now calculate an explicit example to demonstrate the main theorem. We first use Construction 38 to construct an appropriate newform with Eisenstein descent of type $(1,1,1,1,1)$. We thus look for a positive integer $k$, a rational prime $p$, a real quadratic extension $L / \mathbb{Q}$, and an imaginary quadratic extension $K / \mathbb{Q}$ such that $p$ splits in $K$ and such that $p \mid B_{k}$,

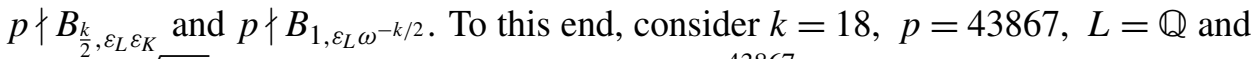
$K=\mathbb{Q}(\sqrt{-5})$. Then 43867 splits in $K$, and $p \mid \frac{43867}{798}=B_{18}=B_{k}$. Furthermore,

$$
B_{9, \varepsilon_{K}}=-5444415378 \equiv 5726(\bmod 43867),
$$

and so $p \nmid B_{\frac{k}{2}, \varepsilon_{L \cdot K}}=B_{\frac{k}{2}, \varepsilon_{K}}$. 
Remark 42. For certain values of $k$, we can simplify the above formula even further. Let $[m]$ denote the smallest nonnegative representative of the residue class $\bmod (p-1)$ of an integer $m$. In the case that $2 \leq[-k / 2] \leq p-4$, by standard congruence theorems (see [Washington 1997, Chapter 5.3], for example), we have

$$
B_{1, \omega^{[-k / 2]}} \equiv \frac{B_{[-k / 2]+1}}{[-k / 2]+1}(\bmod p)
$$

(and hence this congruence also holds $\bmod \lambda \mid p$ ).

Hence, by Remark 42, we have

$$
B_{1, \omega^{-9}} \equiv \frac{B_{43858}}{43858} \equiv 11867(\bmod 43867),
$$

and so $p \nmid B_{1, \varepsilon_{L} \omega^{-k / 2}}=B_{1, \omega^{-9}}$. Hence, applying Construction 38, we get a newform $f_{18} \in S_{18}\left(\mathrm{SL}_{2}(\mathbb{Z})\right)$ such that

$$
f_{18}(q) \equiv \sum_{n=1}^{\infty} \sigma_{17}(n) q^{n}(\bmod \lambda)
$$

for some prime ideal $\lambda \mid p$ of a finite extension over $\mathbb{Q}_{p}$. Note that we can apply the $\left(N_{+}^{+} N_{-}^{-} N_{0}^{0}\right)$-stabilization operator of Construction 40 to obtain a newform $f_{18}^{(N)}$ of weight 18 which has Eisenstein descent of type $\left(1,1, N_{+}, N_{-}, N_{0}\right) \bmod \lambda$. Choose $\left(N_{+}, N_{-}, N_{0}\right)=(7,1,1)$. Then 7 splits in $K=\mathbb{Q}(\sqrt{-5})$. Furthermore,

$$
\Xi(1,1,7,1,1)=1-7^{8} \equiv 25644(\bmod 43867),
$$

and thus $\Xi(1,1,7,1,1) \in \mathbb{Q}$ is not congruent to $0(\bmod \lambda)$.

Let $F / H_{\mathfrak{N}} / K$ be in situation $(S)$ as defined in Section 2.2. Applying Theorem 7, we determine the nontriviality of the associated generalized Heegner cycle

$$
\epsilon_{\left(f_{18}, \mathbb{N}_{K}^{-8}\right)_{/ F}} \Delta\left(\mathbb{N}_{K}^{-8}\right) \in \epsilon_{\left(f_{18}, \mathbb{N}_{K}^{-8}\right)_{/ F}} \mathrm{CH}^{17}\left(X_{16}\right)_{0, E_{f_{18}}}(F) .
$$

Thus we have constructed an algebraic cycle with nontrivial $\left(f_{18}, \mathbb{N}_{K}^{-8}\right)$-isotypic component, whose existence is predicted by the Beilinson-Bloch conjecture since by the Heegner hypothesis we have $L\left(f_{18}, \mathbb{N}_{K}^{8}, 0\right)=0$. (See Sections 2.2 and 3.4 for further details.)

5.4. Application to the Ramanujan $\Delta$ function. Recall the Ramanujan $\Delta$ function, which is a weight- 12 normalized newform of level 1 with $q$-expansion at $\infty$ given by

$$
\Delta(q)=\sum_{n \geq 1} \tau(n) q^{n}
$$


It is well-known that $\tau$ satisfies the congruence $\tau(n) \equiv \sigma_{11}(n)(\bmod 691)$, and so, for any $j \geq 1$ and any quadratic Dirichlet character $\psi$, we have

$$
\left(\theta^{j} \Delta \otimes \psi\right)(q) \equiv\left(\theta^{j} E_{12} \otimes \psi\right)(q)(\bmod 691),
$$

i.e., $\Delta \otimes \psi$ has partial Eisenstein descent over $\mathbb{Q}_{p}$ of type $(\psi, \psi, 1,1,1)$ at 691 . Choosing an auxiliary imaginary quadratic field $K$ satisfying Assumptions 1 with respect to $(681, \Delta \otimes \psi)$ and applying Theorem 7 , we get:

\section{Theorem 43.}

$$
\begin{aligned}
\left(\frac{691^{6}-\psi(691) \tau(691)+691^{5}}{691^{6} \cdot 5 !}\right)^{2}\left(\mathrm{AJ}_{F_{\mathfrak{p}^{\prime}}^{\prime}}\left(\Delta_{\Delta \otimes \psi}\left(\mathbb{N}_{K}^{-5}\right)\right)\left(\omega_{\Delta} \wedge \omega_{A}^{5} \eta_{A}^{5}\right)\right)^{2} \\
\equiv \frac{\left(B_{6, \psi_{0}} B_{1, \psi_{0} \varepsilon_{K} \omega^{-6}}\right)^{2}}{576}\left(\bmod 691 \mathcal{O}_{F^{\prime}}\right),
\end{aligned}
$$

where $\psi_{0}$ is defined as in Definition 6.

Corollary 44. Let $F / H_{\mathfrak{N}} / K$ be in situation $(S)$ as defined in Section 2.2. Suppose $\psi$ is a quadratic character and $K$ is an imaginary quadratic field with odd discriminant $D_{K}<-4$ such that

(1) $691 \nmid \mathfrak{f}(\psi)$,

(2) each prime factor of $691 \cdot \mathfrak{f}(\psi)$ splits in $K$,

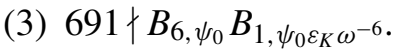

Then

$$
\epsilon_{\left(\Delta \otimes \psi, \mathbb{N}_{K}^{-5}\right)_{/ F}} \Delta_{\Delta \otimes \psi}\left(\mathbb{N}_{K}^{-5}\right) \in \epsilon_{\left(\Delta \otimes \psi, \mathbb{N}_{K}^{-5}\right)_{/ F}} \mathrm{CH}^{11}\left(X_{10}\right)_{0, \mathbb{Q}}(F)
$$

is nontrivial.

To elucidate this result, we include the following table exhibiting a few values of quadratic characters $\psi$ over $\mathbb{Q}$ and imaginary quadratic fields $K$ for which Theorem 43 implies the conclusion of Corollary 44:

\begin{tabular}{lccc}
$K_{\psi}$ & $\mathfrak{f}(\psi)$ & $K$ & $B_{6, \psi_{0}} B_{1, \psi_{0} \varepsilon_{K} \omega^{-6}}(\bmod 691)$ \\
\hline $\mathbb{Q}(\sqrt{3})$ & 12 & $\mathbb{Q}(\sqrt{-23})$ & 583 \\
$\mathbb{Q}(\sqrt{3})$ & 12 & $\mathbb{Q}(\sqrt{-95})$ & 126 \\
$\mathbb{Q}(\sqrt{13})$ & 13 & $\mathbb{Q}(\sqrt{-10})$ & 583 \\
$\mathbb{Q}(\sqrt{-7})$ & -7 & $\mathbb{Q}(\sqrt{-10})$ & 176
\end{tabular}

5.5. Application to elliptic curves. We now focus on the case where $f_{E} \in S_{2}\left(\Gamma_{0}(N)\right)$ is the weight-2 normalized newform associated with an elliptic curve $E / \mathbb{Q}$. If $E[p]$ is reducible, by Theorem 35, $f_{E}$ has Eisenstein descent over $\mathbb{Q}_{p} \bmod p$. We now prove our main application to elliptic curves, which is Theorem 13. 
Proof of Theorem 13. Recall that $f_{E}$ determines an invariant differential $\omega_{f_{E}}=$

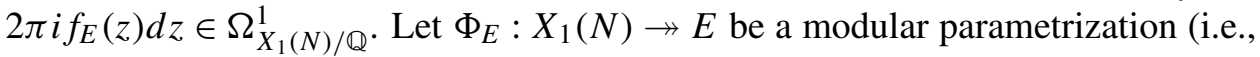
the Eichler-Shimura abelian variety quotient $\Phi_{f_{E}}: X_{1}(N) \rightarrow A_{f_{E}}$, postcomposed with an appropriate isogeny) and let $\omega_{E} \in \Omega_{E / \mathbb{Q}}^{1}$ be an invariant differential chosen so that $\Phi_{E}^{*} \omega_{E}=\omega_{f_{E}}$.

By Theorems 35 and 7 with $k=2$,

$$
\left(\frac{1-a_{p}+p}{p}\right)^{2} \log _{\omega_{E}}^{2} P_{E}(K)=\left(\frac{1-a_{p}+p}{p}\right)^{2} \log _{\omega_{f_{E}}}^{2} P(K)
$$

is congruent to

$$
\frac{\Xi^{2}}{4}\left(\frac{1}{2}\left(1-\psi^{-1}(p)\right) B_{1, \psi_{0}^{-1} \varepsilon_{K}} B_{1, \psi_{0} \omega^{-1}}\right)^{2}\left(\bmod p \mathcal{O}_{K_{\mathfrak{p}}}\right)
$$

if $\psi \neq 1$ and

$$
\frac{\Xi^{2}}{4}\left(\frac{p-1}{p} \log _{p} \bar{\alpha}\right)^{2}\left(\bmod p \mathcal{O}_{K_{\mathfrak{p}}}\right)
$$

if $\psi=1$, where $\psi_{0}$ is defined as in Definition 6 and where

$$
\Xi=\prod_{\ell \mid N_{+}}\left(1-\psi^{-1}(\ell)\right) \prod_{\ell \mid N_{-}}\left(1-\frac{\psi(\ell)}{\ell}\right) \prod_{\ell \mid N_{0}}\left(1-\psi^{-1}(\ell)\right)\left(1-\frac{\psi(\ell)}{\ell}\right) .
$$

(Note that our congruence holds mod $p \mathcal{O}_{K_{\mathrm{p}}}$ because both sides of the congruence are defined over $K_{\mathfrak{p}}$ in this situation.)

Our hypotheses on $(E, p, K)$ and part (2) of Theorem 34 (with $k=2$ ) ensure that none of the terms on the right-hand side of the above congruences vanish $\bmod p$, and hence $\log _{\omega_{E}} P_{E}(K) \neq 0$, i.e., $P_{E}(K)$ is nontorsion.

Remark 45. Suppose $(E, p)$ is as in the statement of Theorem 13, and for simplicity suppose $\psi$ is even and nontrivial. Thus, in particular $E[p](\mathbb{Q})=0$. We will show (Theorem 54) that there always exists an imaginary quadratic $K$ satisfying the appropriate congruence conditions, so that the theorem gives $\operatorname{rank}_{\mathbb{Z}} E(K)=1$. In particular, this implies that we should be able to see that $\operatorname{rank}_{\mathbb{Z}} E(\mathbb{Q}) \leq 1$ a priori from the congruence conditions on $(E, p)$.

Indeed, one can show that $\operatorname{rank}_{\mathbb{Z} / p} \operatorname{Sel}_{p}(E / \mathbb{Q}) \leq 1$ (which implies $\operatorname{rank}_{\mathbb{Z}} E(\mathbb{Q}) \leq 1$ ) through purely algebraic methods. Using standard techniques, one can show that the congruence conditions on $\left(N_{+}, N_{-}, N_{0}\right)$ imply $\operatorname{Sel}_{p}(E / \mathbb{Q}) \subset H^{1}(\mathbb{Q}, E[p] ;\{p\})$. (Here, for $\operatorname{Gal}(\overline{\mathbb{Q}} / \mathbb{Q})$-module $M$ and a finite set $\Sigma$ of places of $\mathbb{Q}, H^{1}(\mathbb{Q}, M ; \Sigma)$ denotes the subgroup of $H^{1}(\mathbb{Q}, M)$ consisting of classes unramified outside $\Sigma$.) See, for example, [Li 2014, Section 2.2] for the case $p=3$ and $E$ semistable; the case for general $p>2$ and general $E$ is completely analogous. The hypothesis $p \nmid B_{1, \psi \omega^{-1}}$ (which is equivalent to $p \nmid L_{p}(\psi, 1)$ since $\psi \neq 1$ and $p \nmid \mathfrak{f}(\psi)$ ) implies, via the main 
theorem of Iwasawa theory over $\mathbb{Q}$ and standard Selmer group control theorems, that $H^{1}\left(\mathbb{Q}, \mathbb{F}_{p}(\psi) ;\{p\}\right)=0$. Since $H^{1}\left(\mathbb{Q}, \mathbb{F}_{p}(\psi) ; \varnothing\right) \subset H^{1}\left(\mathbb{Q}, \mathbb{F}_{p}(\psi) ;\{p\}\right)$, we have $H^{1}\left(\mathbb{Q}, \mathbb{F}_{p}(\psi) ; \varnothing\right)=0$, which in turn implies that $H^{1}\left(\mathbb{Q}, \mathbb{F}_{p}\left(\psi^{-1} \omega\right) ;\{p\}\right)=\mathbb{Z} / p$ (see, for example, loc. cit. Proposition 2.13). Now if $\mathbb{F}_{p}(\psi) \subset E[p]$, from the standard long exact sequence of cohomology (see Section 2.3 of loc. cit., for example), one obtains a map $\phi: \operatorname{Sel}_{p}(E / \mathbb{Q}) \rightarrow H^{1}\left(\mathbb{Q}, \mathbb{F}_{p}\left(\psi^{-1} \omega\right)\right)$ such that $\operatorname{ker}(\phi) \subset H^{1}\left(\mathbb{Q}, \mathbb{F}_{p}(\psi) ;\{p\}\right)=0$ and $\operatorname{im}(\phi) \subset H^{1}\left(\mathbb{Q}, \mathbb{F}_{p}\left(\psi^{-1} \omega\right) ;\{p\}\right)=\mathbb{Z} / p$. Thus we get $\operatorname{Sel}_{p}(E / \mathbb{Q}) \subset \operatorname{im}(\phi) \subset H^{1}\left(\mathbb{Q}, \mathbb{F}_{p}\left(\psi^{-1} \omega\right) ;\{p\}\right)=\mathbb{Z} / p$. If, on the other hand, $\mathbb{F}_{p}\left(\psi^{-1} \omega\right) \subset E[p]$, one obtains $\phi: \operatorname{Sel}_{p}(E / \mathbb{Q}) \rightarrow H^{1}\left(\mathbb{Q}, \mathbb{F}_{p}(\psi)\right)$ with $\operatorname{ker}(\phi) \subset H^{1}\left(\mathbb{Q}, \mathbb{F}_{p}\left(\psi^{-1} \omega\right) ;\{p\}\right)=\mathbb{Z} / p$ and $\operatorname{im}(\phi) \subset H^{1}\left(\mathbb{Q}, \mathbb{F}_{p}(\psi) ;\{p\}\right)=0$. Thus we get $\operatorname{Sel}_{p}(E / \mathbb{Q}) \subset \operatorname{ker}(\phi) \subset H^{1}\left(\mathbb{Q}, \mathbb{F}_{p}\left(\psi^{-1} \omega\right) ;\{p\}\right)=\mathbb{Z} / p$.

Remark 46. Since the congruence in the proof of Theorem 13 comes from $p$-adic interpolation of $p$-adically integral period sums, we should be able to see a priori that both sides of the congruence are $p$-adically integral. This is self-evident for the right-hand side, and also a priori true for the left-hand side as follows. Note that since $p \nmid N$, we have $p+1-a_{p}=\left|\tilde{E}\left(\mathbb{F}_{p}\right)\right|$ by the Eichler-Shimura relation. Let $\hat{E}$ denote the formal group of $E$, so that $\hat{E}\left(\mathfrak{p} \mathcal{O}_{K_{\mathfrak{p}}}\right)$ has index $\left|\tilde{E}\left(\mathbb{F}_{p}\right)\right|$ in $E\left(K_{\mathfrak{p}}\right)$. (Recall that $\mathfrak{p}$ is the previously fixed prime above $p$ determined by our embedding $\left.K \hookrightarrow \overline{\mathbb{Q}}_{p}.\right)$ Then $\left[1-a_{p}+p\right] P_{E}(K)=\left[\left|\tilde{E}\left(\mathbb{F}_{p}\right)\right|\right] P_{E}(K) \in \hat{E}\left(\mathfrak{p} \mathcal{O}_{K_{\mathfrak{p}}}\right)$.

Suppose for the moment that $X_{1}(N) \rightarrow E$ is optimal (i.e., $E$ is the strong Weil curve in its isogeny class). Well-known results due to Mazur [1978] on the Manin constant $c(E)$ imply that if $\ell \mid c(E)$, then $\ell^{2} \mid 4 N$. Thus for $p$ odd of good reduction, we have $p \nmid c(E)$. Thus letting $\log _{E}$ denote the canonical formal logarithm on $E$ (i.e., the formal logarithm arising from the unique normalized invariant differential on $E$ ), we have $\log _{\omega_{E}} T=c(E)^{-1} \log _{E} T$, meaning our normalization of the formal logarithm does not change the $p$-divisibility on the formal group. That is, $\log _{\omega_{E}} \hat{E}\left(\mathfrak{p} \mathcal{O}_{K_{\mathfrak{p}}}\right) \subset \mathfrak{p} \mathcal{O}_{K_{\mathfrak{p}}}$. Thus by the previous paragraph, $\left(\left(1-a_{p}+p\right) / p\right) \log _{\omega_{E}} P_{E}(K)$ is $p$-adically integral.

For $E$ nonoptimal, the choice of modular parametrization might change the normalization of the formal logarithm, since we are postcomposing the Eichler-Shimura projection with a $\mathbb{Q}$-isogeny which does not necessarily preserve normalizations of the formal logarithm. This can still be shown to not affect $p$-adic integrality of $\log _{\omega_{E}}$ on $\hat{E}\left(\mathfrak{p} \mathcal{O}_{K_{\mathfrak{p}}}\right)$.

Remark 47. Suppose that $(E, p, K)$ is as in the hypotheses of Theorem 13. One can show that $\log _{\omega_{E}} P_{E}(K) \equiv 0(\bmod p)$ as follows. Let $F=K\left(\mu_{p}\right)$, and choose a prime $\pi \mid p$ of $F$; note that $\mathfrak{p}$ is totally ramified in $K\left(\mu_{p}\right)$, so that $\mathcal{O}_{F_{\pi}} / \pi \cong \mathbb{F}_{p}$ and $\operatorname{ord}_{\pi}(p)=p-1$. If $P_{K}(E)$ is torsion, then $\log _{\omega_{E}} P_{E}(K)=0$ by properties of the formal logarithm (see [Silverman 2009, Chapter 4]), so assume that $P_{E}(K)$ is nontorsion. Suppose $\psi \neq 1$. Then $\left|\tilde{E}\left(\mathbb{F}_{p}\right)\right|-(1+p)=-a_{p} \equiv-\psi(p) \neq-1(\bmod p)$ 
implies $\left|\tilde{E}\left(\mathbb{F}_{p}\right)\right| \not \equiv 0(\bmod p)$. Therefore, $\hat{E}\left(\pi \mathcal{O}_{F_{\pi}}\right)$ has index prime to $p$ in $E\left(F_{\pi}\right)$, and so $\log _{\omega_{E}} E\left(F_{\pi}\right) \subset \pi \mathcal{O}_{F_{\pi}}$, and $\log _{\omega_{E}} P_{E}(K) \in \mathfrak{p} \mathcal{O}_{K_{\mathfrak{p}}}$.

Now suppose $\psi=1$, so that $\left|\tilde{E}\left(\mathbb{F}_{p}\right)\right|-(1+p)=-a_{p} \equiv-\psi(p)=-1(\bmod p)$, and so $\left|\tilde{E}\left(\mathbb{F}_{p}\right)\right| \equiv 0(\bmod p)$. Moreover, $p$ is ordinary good reduction and so $|\tilde{E}(\mathbb{F})[p]|=\left|\tilde{E}\left(\mathbb{\mathbb { F }}_{p}\right)\right|=p$ implies $p|| \tilde{E}\left(\mathbb{F}_{p}\right) \mid$. Then, by Theorem 35, we have $E[p]=E(F)[p]$, and so the exact sequence

$$
0 \rightarrow \hat{E}\left(\pi \mathcal{O}_{F_{\pi}}\right) \rightarrow E\left(F_{\pi}\right) \rightarrow \tilde{E}\left(\mathbb{F}_{p}\right) \rightarrow 0
$$

splits, i.e., $E\left(F_{\pi}\right)=\hat{E}\left(\pi \mathcal{O}_{F_{\pi}}\right) \oplus \tilde{E}\left(\mathbb{F}_{p}\right)$, and moreover $\tilde{E}\left(\mathbb{F}_{p}\right) \subset E\left(F_{\pi}\right)^{\text {tor }}$. (The torsion of $E\left(F_{\pi}\right)$ that is outside of $p$ injects into $\tilde{E}\left(\mathbb{F}_{p}\right)$, and $\left.\tilde{E}\left(\mathbb{F}_{p}\right)[p] \subset E\left(F_{\pi}\right)[p]\right)$.) Thus since $\log _{\omega_{E}} \hat{E}\left(\pi \mathcal{O}_{F_{\pi}}\right) \subset \pi \mathcal{O}_{F_{\pi}}$ and $\log _{\omega_{E}} \tilde{E}\left(\mathbb{F}_{p}\right)=0$, we have $\log _{\omega_{E}} P_{E}(K) \in \pi \mathcal{O}_{F_{\pi}}$. Now since $P_{E}(K) \in E(K) \subset E\left(K_{\mathfrak{p}}\right)$, we have $\log _{\omega_{E}} P_{E}(K) \in K_{\mathfrak{p}} \cap \pi \mathcal{O}_{F_{\pi}}=\mathfrak{p} \mathcal{O}_{K_{\mathfrak{p}}}$.

Remark 48. While the proof of Theorem 13 accounts for the case $\psi=1$, it gives no new information since the left side of the relevant special value congruence is always $0(\bmod p)$ : Remark 47 shows that $\log _{\omega_{E}} P_{E}(K) \equiv 0(\bmod p)$, and $a_{p} \equiv$ $\psi(p)=1(\bmod p)$ implies that $\left(1-a_{p}+p\right) / p$ is a unit in $\mathcal{O}_{\mathbb{C}_{p}}$. Note that this forces $\Xi\left(1, N_{\text {split }}, N_{\text {nonsplit }}, N_{\text {add }}\right) \equiv 0(\bmod p)$.

In fact, if $\psi=1$, one can show that, for any elliptic curve, $N_{\text {split }} N_{\text {add }} \neq 1$ in the following way: assume $N_{\text {add }}=1$, so that $N$ is squarefree. A theorem of Ribet then shows that $N_{\text {split }} \neq 1$ (see the Ph.D. thesis of Hwajong Yoo [2015, Theorem 2.2] and the ensuing remark therein). Thus if $\psi=1$, we have $\Xi\left(1, N_{\text {split }}, N_{\text {nonsplit }}, N_{\text {add }}\right)=0$.

When $\psi \neq 1$, we have $a_{p} \equiv \psi(p) \neq 1(\bmod p)$, and so the factor of $\left(1-a_{p}+p\right) / p$ is congruent to (unit) $/ p(\bmod p)$, thus canceling out a $p$-divisibility of $\log _{\omega_{E}} P_{E}(K)$ in the special value congruence.

Remark 49. Suppose we are in the situation of Theorem 13, so in particular $p \nmid N$ is a good prime which is split in $K$. Let $\varpi$ be a local uniformizer at $\mathfrak{p}$. (Recall that $\mathfrak{p}$ is the prime above $p$ determining the embedding $K \hookrightarrow \overline{\mathbb{Q}}_{p}$.) Then as $1-a_{p}+p=\left|\tilde{E}\left(\mathbb{F}_{p}\right)\right|$, we have that $P:=\left[1-a_{p}+p\right] P_{E}(K)$ belongs to the formal group $\hat{E}\left(\mathfrak{p} \mathcal{O}_{K_{\mathfrak{p}}}\right)$. One can show that $\log _{E} P \in \varpi \mathcal{O}_{K_{\mathfrak{p}}}^{\times}=p \mathcal{O}_{K_{\mathfrak{p}}}^{\times}$if and only if the image of $P$ in $E\left(K_{\mathfrak{p}}\right) / p E\left(K_{\mathfrak{p}}\right)$ is not in the image of $E\left(K_{\mathfrak{p}}\right)[p]$ as follows. If $P$ is not in the image of $E\left(K_{\mathfrak{p}}\right)[p]$ in $E\left(K_{\mathfrak{p}}\right) / p E\left(K_{\mathfrak{p}}\right)$, then suppose $\log _{E} P \notin \varpi \mathcal{O}_{K_{\mathfrak{p}}}^{\times}$. Since $P \in \hat{E}\left(\mathfrak{p} \mathcal{O}_{K_{\mathfrak{p}}}\right)$, we know that $P$ is either torsion (i.e., $\log _{E} P=0$ ) or $P \in \mathfrak{p}^{2} \mathcal{O}_{K_{\mathfrak{p}}}$ (i.e., $\left.\log _{E} P \in \mathfrak{p}^{2} \mathcal{O}_{K_{\mathfrak{p}}}\right)$. However, in the first case this implies $P \in E\left(K_{\mathfrak{p}}\right)[p]$ (since $\hat{E}\left(\mathfrak{p} \mathcal{O}_{K_{\mathfrak{p}}}\right)$ has no $p$-torsion) and in the second it implies $P \in \hat{E}\left(\mathfrak{p}^{2} \mathcal{O}_{K_{\mathfrak{p}}}\right)$; thus $P$ is $p$-divisible. Therefore, $P \in \hat{E}\left(\mathfrak{p} \mathcal{O}_{K_{\mathfrak{p}}}\right)+p E\left(K_{\mathfrak{p}}\right)$, a contradiction. Conversely, if $\log _{E} P \in \varpi \mathcal{O}_{K_{\mathfrak{p}}}^{\times}$, then we have $P \notin E\left(K_{\mathfrak{p}}\right)[p]+p E\left(K_{\mathfrak{p}}\right)$; otherwise, we have $P \in E\left(K_{\mathfrak{p}}\right)[p]+p \hat{E}\left(\mathfrak{p} \mathcal{O}_{K_{\mathfrak{p}}}\right)$ (since a priori $P \in \hat{E}\left(\mathfrak{p} \mathcal{O}_{K_{\mathfrak{p}}}\right)$ ); thus $\log _{E} P \in \mathfrak{p}^{2} \mathcal{O}_{K_{\mathfrak{p}}}$, a contradiction. 
Thus the nonvanishing results of $\log _{E} P \bmod p$ provided by Theorem 13 give information on local $p$-divisibility of $P$ (and thus also of $P_{E}(K)$ ). Note that if $p \geq 3$, then $\hat{E}\left(\mathfrak{p} \mathcal{O}_{K_{\mathfrak{p}}}\right)[p]=0$, and so $E\left(K_{\mathfrak{p}}\right)[p] \hookrightarrow \tilde{E}\left(\mathbb{F}_{p}\right)[p]$. In particular, if $p$ is of supersingular reduction, then $\tilde{E}\left(\mathbb{F}_{p}\right)[p]=0$, so $E\left(K_{\mathfrak{p}}\right)[p]=0$ and the nonvanishing of $\log _{E} P \bmod p$ is equivalent to $P$ having nontrivial image in $E\left(K_{\mathfrak{p}}\right) / p E\left(K_{\mathfrak{p}}\right)$, i.e., the condition that $P$ not be $p$-divisible in $E\left(K_{\mathfrak{p}}\right)$.

For any elliptic curve $E$ over $\mathbb{Q}$, let $w(E)$ denote the global root number, which factors as a product of local root numbers

$$
w(E)=w_{\infty}(E) \prod_{\ell<\infty} w_{\ell}(E) .
$$

Proposition 50. Suppose that $(E, p)$ is as in Theorem 13 and that $\psi$ is a quadratic character. If either

(1) $p \geq 5$, or

(2) $E=E^{\prime} \otimes \psi$ for some semistable elliptic curve $E^{\prime}$ of conductor coprime to $\mathfrak{f}(\psi)$, then $w(E)=-\psi(-1)$. Thus, $\operatorname{rank}_{\mathbb{Z}} E(\mathbb{Q})=\frac{1}{2}(1+\psi(-1))$, and $\operatorname{rank}_{\mathbb{Z}} E_{K}(\mathbb{Q})=$ $\frac{1}{2}(1-\psi(-1))$ for any imaginary quadratic $K$ as in Theorem 13.

Proof. By our assumptions in the statement of Theorem 13, $|\mathfrak{f}(\psi)|^{2} \mid N_{\text {add }}$ and $N_{\text {split }}=1$. By standard properties of the root number (see [Dokchitser 2013, Section 3.4]), we have

(1) if $\ell \| N$ (i.e., $\ell \mid N_{\text {nonsplit }}$ ), then $w_{\ell}(E)=1$,

(2) $w_{\infty}(E)=-1$.

Hence,

$$
w(E)=-\prod_{\ell \mid N_{\text {add }}} w_{\ell}(E) .
$$

Suppose first that (1) in the statement of the proposition holds, i.e., $p \geq 5$. By Theorem 34, $\bar{\rho}_{f_{E}} \cong \mathbb{F}_{p} \oplus \mathbb{F}_{p}(\omega)$. Hence $\bar{\rho}_{f_{E}} \cong \mathbb{F}_{p}(\psi) \oplus \mathbb{F}_{p}\left(\psi^{-1} \omega\right)$ and thus $E$ admits a degree $p$ isogeny $\phi: E \rightarrow E^{\prime}$. Since $p \geq 5$, by [Dokchitser 2013, Theorem 3.25], for $\ell \mid N_{\text {add }}$ we have

$$
w_{\ell}(E)=\left(-1, F / \mathbb{Q}_{\ell}\right)
$$

where $F:=\mathbb{Q}_{\ell}$ (coordinates of points in $\left.\operatorname{ker}(\phi)\right)$, and where $\left(\cdot, F / \mathbb{Q}_{\ell}\right)$ is the norm residue symbol. By our assumptions, we see that $F=\mathbb{Q}_{\ell}\left(\mu_{2 p}, \sqrt{\psi(-1)|\mathfrak{f}(\psi)|}\right)$, which is ramified only if $\ell \mid p \mathfrak{f}(\psi)$. Thus $\mathbb{Q}_{\ell}\left(\mu_{2 p}\right) / \mathbb{Q}_{\ell}$ is an unramified extension. Thus, by class field theory, $\left(-1, F / \mathbb{Q}_{\ell}\right)=\left(-1, \mathbb{Q}_{\ell}(\sqrt{\psi(-1)|\mathfrak{f}(\psi)|}) / \mathbb{Q}_{\ell}\right)=\psi_{\ell}(-1)$, and so $w_{\ell}(E)=\psi_{\ell}(-1)$. Hence, in all, we have

$$
w(E)=-\prod_{\ell \mid N_{\text {add }}} w_{\ell}(E)=-\prod_{\ell \mid N_{\text {add }}} \psi_{\ell}(-1)=-\psi(-1),
$$


where the last equality follows since $\mathfrak{f}(\psi) \mid N_{\text {add }}$.

Now suppose that (2) in the statement of the proposition holds. Let $N^{\prime}$ denote the conductor of $E^{\prime}$. By the computations of [Balsam 2014, Proposition 1] describing the changes of local root numbers under quadratic twists,

(1) if $\ell \nmid N^{\prime} \mathfrak{f}(\psi)$ then $w_{\ell}(E)=1$,

(2) if $\ell \mid N^{\prime}$ (so that $\ell \nmid \mathfrak{f}(\psi)$ ) then $w_{\ell}(E)=w_{\ell}\left(E^{\prime}\right) \psi_{\ell}(\ell)=-a_{\ell}\left(E^{\prime}\right) \psi_{\ell}(\ell)$,

(3) if $\ell \mid \mathfrak{f}(\psi)$ (so that $\ell \nmid N^{\prime}$ ) then $w_{\ell}(E)=w_{\ell}\left(E^{\prime}\right) \psi_{\ell}(-1)=\psi_{\ell}(-1)$.

Hence by our assumptions in the statement of Theorem 13 and Remark 15, we have $w(E)=-\psi(-1) \prod_{\ell \mid N^{\prime}}\left(-a_{\ell}\left(E^{\prime}\right) \psi_{\ell}(\ell)\right)=-\psi(-1)$. Putting this together, we compute $w(E)=-\psi(-1)$.

Now, by Theorem 13 and parity considerations, we immediately get $\operatorname{rank}_{\mathbb{Z}} E(\mathbb{Q})=$ $\frac{1}{2}(1+\psi(-1))$ and $\operatorname{rank}_{\mathbb{Z}} E_{K}(\mathbb{Q})=\frac{1}{2}(1-\psi(-1))$ for any $K$ as in Theorem 13 .

5.6. Calculating ranks in positive density subfamilies of quadratic twists. In the case $p=3$, the Teichmïller character $\omega$ is quadratic and is in fact the character associated with the imaginary quadratic field $\mathbb{Q}(\sqrt{-3})$. Thus we have (using $B_{1, \psi \omega^{-1}}=-L\left(\psi \omega^{-1}, 0\right)$, the functional equation, and the class number formula),

$$
B_{1, \psi \omega^{-1}}=2 \frac{h_{K_{\psi} \cdot \mathbb{Q}(\sqrt{-3})}}{\mid \mathcal{O}_{K_{\psi} \cdot \mathbb{Q}(\sqrt{-3})}^{\times}} .
$$

Hence our nondivisibility criterion involving Bernoulli numbers in Theorem 13 reduces to the non-3-divisibility of the class numbers of a pair of imaginary quadratic fields, and is thus amenable to class number 3-divisibility results in the tradition of [Davenport and Heilbronn 1971].

For the remainder of this section, suppose $p=3$ and suppose we are given $E / \mathbb{Q}$ of conductor $N=N_{\text {split }} N_{\text {nonsplit }} N_{\text {add }}$ such that $E$ [3] is reducible of type $\left(1,1, N_{\text {split }}, N_{\text {nonsplit }}, N_{\text {add }}\right)$. Let $L=K_{\psi}$, and furthermore suppose that $L$ satisfies the congruence conditions

(1) 3 is inert in $L$,

(2) $3 \nmid h_{L \cdot \mathbb{Q}(\sqrt{-3})}$,

(3) if $\ell \mid N_{\text {split }}$, then $\ell$ is inert or ramified in $L$,

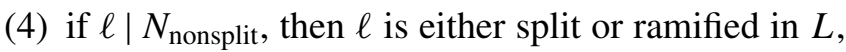

(5) if $\ell \mid N_{\text {add }}$, then $\ell$ is either inert in $L$ and $\ell \not \equiv 2(\bmod 3)$, or $\ell$ is ramified in $L$.

Then we can apply Theorem 13 and Proposition 50 to the curve $E \otimes \psi$ (using the fact that $a_{\ell}(E \otimes \psi)=\psi(\ell) a_{\ell}(E)$, so that, by conditions (2) and (3), $a_{\ell}(E \otimes \psi)=-1$ for $\left.\ell \mid N_{\text {split }} N_{\text {nonsplit }}\right)$, thus obtaining $\operatorname{rank}_{\mathbb{Z}}(E \otimes \psi)(\mathbb{Q})=\frac{1}{2}(1+\psi(-1))$. Using results of [Nakagawa and Horie 1988] and [Taya 2000] regarding 3-divisibilities 
of class numbers of quadratic fields, we can produce a positive proportion of real quadratic $L$ as above, thus showing that a positive proportion of quadratic twists of $E$ have rank $\frac{1}{2}(1+\psi(-1))$ over $\mathbb{Q}$.

Using these same class number divisibility results, we can also produce a positive proportion of imaginary quadratic $K$ such that

(1) 3 is split in $K$,

(2) $3 \nmid h_{L \cdot K}$,

(3) $K$ satisfies the Heegner hypothesis with respect to $E$,

and thus, using Theorem 13 and Proposition 50, we can show that a positive proportion of imaginary quadratic twists $E_{K}$ of $E$ have $\operatorname{rank}_{\mathbb{Z}} E_{K}(\mathbb{Q})=\frac{1}{2}(1-\psi(-1))$.

To this end, let us recall the result of Horie and Nakagawa. For any $x \geq 0$, let $K^{+}(x)$ denote the set of real quadratic fields $k$ with fundamental discriminant $D_{k}<x$ and $K^{-}(x)$ the set of imaginary quadratic fields $k$ with fundamental discriminant $\left|D_{k}\right|<x$. Set

$$
\begin{aligned}
& K^{+}(x, m, M):=\left\{k \in K^{+}(x): D_{k} \equiv m(\bmod M)\right\}, \\
& K^{-}(x, m, M):=\left\{k \in K^{-}(x): D_{k} \equiv m(\bmod M)\right\} .
\end{aligned}
$$

Moreover, for a quadratic field $k$, we denote by $h_{k}[3]$ the number of ideal classes of $k$ whose cubes are principal (i.e., the order of 3-torsion of the ideal class group).

Theorem 51 [Nakagawa and Horie 1988]. Suppose that $m$ and $M$ are positive integers such that if $\ell$ is an odd prime number dividing $(m, M)$, then $\ell^{2}$ divides $M$ but not $m$. Further, if $M$ is even, suppose that

(1) $4 \mid M$ and $m \equiv 1(\bmod 4)$, or

(2) $16 \mid M$ and $m \equiv 8$ or $12(\bmod 16)$.

Then

$$
\begin{aligned}
\sum_{k \in K^{+}(x, m, M)} h_{k}[3] \sim \frac{4}{3}\left|K^{+}(x, m, M)\right| & (x \rightarrow \infty), \\
\sum_{k \in K^{-}(x, m, M)} h_{k}[3] \sim 2\left|K^{-}(x, m, M)\right| & (x \rightarrow \infty) .
\end{aligned}
$$

Furthermore,

$$
\left|K^{+}(x, m, M)\right| \sim\left|K^{-}(x, m, M)\right| \sim \frac{3 x}{\pi^{2} \Phi(M)} \prod_{\ell \mid M} \frac{q}{\ell+1} \quad(x \rightarrow \infty) .
$$

Here $f(x) \sim g(x)(x \rightarrow \infty)$ means that $\lim _{x \rightarrow \infty} f(x) / g(x)=1$, $\ell$ ranges over primes dividing $M, q=4$ if $\ell=2$, and $q=\ell$ otherwise. 
Now set

$$
\begin{aligned}
& K_{*}^{+}(x, m, M):=\left\{k \in K^{+}(x, m, M): h_{k}[3]=1\right\}, \\
& K_{*}^{-}(x, m, M):=\left\{k \in K^{-}(x, m, M): h_{k}[3]=1\right\} .
\end{aligned}
$$

Taya's argument [2000] for estimating $\left|K_{*}^{ \pm}(x, m, M)\right|$ goes as follows. Since $h_{k}[3] \geq 3$ if $h_{k}[3] \neq 1$, we have the bound

$$
\left|K_{*}^{ \pm}(x, m, M)\right|+3\left(\left|K^{ \pm}(x, m, M)\right|-\left|K_{*}^{ \pm}(x, m, M)\right|\right) \leq \sum_{k \in K^{ \pm}(x, m, M)} h_{k}[3] .
$$

Hence,

$$
\left|K_{*}^{ \pm}(x, m, M)\right| \geq \frac{3}{2}\left|K^{ \pm}(x, m, M)\right|-\frac{1}{2} \sum_{k \in K^{ \pm}(x, m, M)} h_{k}[3] .
$$

Now, by Theorem 51, we have

$$
\begin{array}{ll}
\frac{3}{2}\left|K^{+}(x, m, M)\right|-\frac{1}{2} \sum_{k \in K^{+}(x, m, M)} h_{k}[3] \sim \frac{5}{6}\left|K^{+}(x, m, M)\right| & (x \rightarrow \infty), \\
\frac{3}{2}\left|K^{-}(x, m, M)\right|-\frac{1}{2} \sum_{k \in K^{-}(x, m, M)} h_{k}[3] \sim \frac{1}{2}\left|K^{-}(x, m, M)\right| \quad(x \rightarrow \infty),
\end{array}
$$

and hence,

$$
\begin{aligned}
& \lim _{x \rightarrow \infty} \frac{\left|K_{*}^{+}(x, m, M)\right|}{x} \geq \frac{5}{2 \pi^{2} \Phi(M)} \prod_{\ell \mid M} \frac{q}{\ell+1}, \\
& \lim _{x \rightarrow \infty} \frac{\left|K_{*}^{-}(x, m, M)\right|}{x} \geq \frac{3}{2 \pi^{2} \Phi(M)} \prod_{\ell \mid M} \frac{q}{\ell+1} .
\end{aligned}
$$

Thus, we have:

Proposition 52. Suppose $m$ and $M$ satisfy the conditions of Theorem 51. Then

$$
\begin{aligned}
& \lim _{x \rightarrow \infty} \frac{\left|K_{*}^{+}(x, m, M)\right|}{\left|K^{+}(x, 1,1)\right|} \geq \frac{5}{6 \Phi(M)} \prod_{\ell \mid M} \frac{q}{\ell+1}, \\
& \lim _{x \rightarrow \infty} \frac{\left|K_{*}^{-}(x, m, M)\right|}{\left|K^{-}(x, 1,1)\right|} \geq \frac{1}{2 \Phi(M)} \prod_{\ell \mid M} \frac{q}{\ell+1},
\end{aligned}
$$

where $q=4$ if $\ell=2$ and $q=\ell$ otherwise. In particular, the set of real (resp. imaginary) quadratic fields $k$ such that $D_{k} \equiv m(\bmod M)$ and $3 \nmid h_{k}$ has positive density in the set of all real (resp. imaginary) quadratic fields.

Proof. This follows from the above asymptotic estimates and the fact that we have $\left|K^{ \pm}(x, 1,1)\right| \sim 3 x / \pi^{2}$ by Theorem 51 .

We are now ready to prove our positive density results. For a quadratic field $L$, let $E_{L}$ denote the quadratic twist of $E$ by $L$. 
Theorem 53. Suppose ( $\left.N_{\mathrm{split}}, N_{\text {nonsplit }}, N_{\mathrm{add}}\right)$ is a triple of pairwise coprime integers such that $N_{\text {split }} N_{\text {nonsplit }}$ is squarefree, $N_{\text {add }}$ is squarefull and $N_{\text {split }} N_{\text {nonsplit }} N_{\text {add }}$ equals $N$. If $2 \nmid N$ let $M^{\prime}=N$, if $2 \| N$ let $M^{\prime}=\operatorname{lcm}(N, 8)$, and if $4 \mid N$ let $M^{\prime}=\operatorname{lcm}(N, 16)$. Then a proportion of at least

$$
\frac{1}{12 \Phi\left(M^{\prime}\right)} \prod_{\substack{\ell \mid N_{\text {split }} N_{\text {nonsplit }} \\
\ell \text { odd }}} \frac{1}{\begin{array}{c}
\ell \mid N_{\text {add }} \\
\ell \text { odd } \\
\ell \equiv 1(\bmod 3)
\end{array}} \prod_{\begin{array}{c}
\ell \mid N_{\text {add }} \\
\ell \text { odd } \\
\ell \equiv 2(\bmod 3)
\end{array}}(\ell) \prod_{4\left|N_{\text {add }} \ell\right| 3 M^{\prime}} 2 \prod \frac{q}{\ell+1}
$$

real quadratic extensions $L / \mathbb{Q}$ satisfy

(1) 3 is inert in $L$,

(2) $3 \nmid h_{L \cdot \mathbb{Q}(\sqrt{-3})}$,

(3) $\ell \mid N_{\text {split }}$ implies $\ell$ is inert in $L$,

(4) $\ell \mid N_{\text {nonsplit }}$ implies $\ell$ is split in $L$,

(5) $\ell \mid N_{\text {add }}$ implies $\ell$ is inert in $L$ and $\ell \not \equiv 2(\bmod 3)$, or $\ell$ is ramified in $L$,

(6) $4 \mid N$ implies $D_{L} \equiv 8$ or $12(\bmod 16)$.

Moreover, a proportion of at least

$$
\frac{1}{4 \Phi\left(M^{\prime}\right)} \prod_{\substack{\ell \mid N_{\text {split }} N_{\text {nonsplit }} \\
\ell \text { odd }}} \frac{1}{2}(\ell-1) \prod_{\begin{array}{c}
\ell \mid N_{\text {add }} \\
\ell \text { odd } \\
\ell \equiv 1(\bmod 3)
\end{array}} \frac{1}{2}(\ell+2)(\ell-1) \prod_{\begin{array}{c}
\ell \mid N_{\text {add }} \\
\ell \text { odd } \\
\ell \equiv 2(\bmod 3)
\end{array}}(\ell-1) \prod_{4\left|N_{\text {add }} \ell\right| 3 M^{\prime}} 2 \prod \frac{q}{\ell+1}
$$

imaginary quadratic extensions $L / \mathbb{Q}$ satisfy

(1) 3 is inert in $L$,

(2) $3 \nmid h_{L}$,

(3) $\ell \mid N_{\text {split }}$ implies $\ell$ is inert in $L$,

(4) $\ell \mid N_{\text {nonsplit }}$ implies $\ell$ is split in $L$,

(5) $\ell \mid N_{\text {add }}$ implies $\ell$ is inert in $L$ and $\ell \not \equiv 2(\bmod 3)$, or $\ell$ is ramified in $L$,

(6) $4 \mid N$ implies $D_{L} \equiv 8$ or $12(\bmod 16)$.

(Here, again, $q=4$ for $\ell=2$, and $q=\ell$ for odd primes $\ell$.)

Proof. We seek to apply Proposition 52. Let $M=9 N$ if $2 \nmid N$, let $M=91 \mathrm{~cm}(N, 8)$ if $2 \| N$, and let $M=9 \operatorname{lcm}(N, 16)$ if $4 \mid N$. Using the Chinese remainder theorem, choose a positive integer $m$ such that

(1) $m \equiv 3(\bmod 9)$,

(2) $\ell$ odd prime, $\ell \mid N_{\text {split }} \Rightarrow m \equiv-3$ [quadratic nonresidue unit] ( $\bmod \ell$ ),

(3) $2 \mid N_{\text {split }} \Rightarrow m \equiv 1(\bmod 8)$, 
(4) $\ell$ odd prime, $\ell \mid N_{\text {nonsplit }} \Rightarrow m \equiv-3$ [quadratic residue unit] ( $\left.\bmod \ell\right)$,

(5) $2 \mid N_{\text {nonsplit }} \Longrightarrow m \equiv 5(\bmod 8)$,

(6) $\ell$ odd prime, $\ell \mid N_{\text {add }}, \ell \equiv 1(\bmod 3) \Longrightarrow m \equiv-3$ [quadratic nonresidue unit] $(\bmod \ell)$ or $m \equiv 0(\bmod \ell)$ and $m \neq \equiv 0\left(\bmod \ell^{2}\right)$,

(7) $\ell$ odd prime, $\ell \mid N_{\text {add }}, \ell \equiv 2(\bmod 3) \Longrightarrow m \equiv 0(\bmod \ell)$ and $m \neq \equiv\left(\bmod \ell^{2}\right)$,

(8) $4 \mid N_{\text {add }} \Longrightarrow m \equiv 8$ or $12(\bmod 16)$.

Suppose $L$ is any real quadratic field with fundamental discriminant $D_{L}$ and $-3 D_{L} \equiv m(\bmod M)$. Then the above congruence conditions on $m$ along with our assumptions imply

(1) 3 is inert in $L$,

(2) $\ell$ prime, $\ell \mid N_{\text {split }} \Rightarrow \ell$ is inert in $L$,

(3) $\ell$ prime, $\ell \mid N_{\text {nonsplit }} \Rightarrow \ell$ is split in $L$,

(4) $\ell$ odd prime, $\ell \mid N_{\text {add }}, \ell \equiv 1(\bmod 3) \Longrightarrow \ell$ is inert or ramified in $L$,

(5) $\ell$ odd prime, $\ell \mid N_{\text {add }}, \ell \equiv 2(\bmod 3) \Longrightarrow \ell$ is ramified in $L$,

(6) $4 \mid N_{\text {add }} \Longrightarrow 2$ is ramified in $L$,

(7) if $2 \| N$, then $4 \mid M$ and $m \equiv 1(\bmod 4)$,

(8) if $4 \mid N$, then $16 \mid M$ and $m \equiv 8$ or $12(\bmod 16)$.

Thus for real quadratic $L$ such that $D_{L \cdot \mathbb{Q}(\sqrt{-3})}=-3 D_{L} \equiv m(\bmod M), L$ satisfies all the desired congruence conditions except for possibly $3 \nmid h_{L \cdot \mathbb{Q}(\sqrt{-3})}$. Moreover, the congruence conditions above imply that $m$ and $M$ are valid positive integers for Theorem 51 (in particular implying that $4 \mid D_{L}$ if $4 \mid N$ ). (Note that in congruence conditions (2) and (3) above, we do not allow $m \equiv 0(\bmod \ell)$, i.e., $\ell$ ramified in $L$, because the resulting pair $m$ and $M$ would violate the auxiliary hypothesis of Theorem 51.) Thus, by Proposition 52,

$$
\lim _{x \rightarrow \infty} \frac{\left|K_{*}^{-}(x, m, M)\right|}{\left|K^{-}(x, 1,1)\right|} \geq \frac{1}{2 \Phi(M)} \prod_{\ell \mid M} \frac{q}{\ell+1},
$$

so a positive proportion of real quadratic $L$ satisfy $D_{L \cdot \mathbb{Q}(\sqrt{-3})}=-3 D_{L} \equiv m(\bmod M)$ and $3 \nmid h_{L \cdot \mathbb{Q}(\sqrt{-3})}$. Moreover, noticing that the congruence conditions (1)-(6) on $m$ above are independent (again by the Chinese remainder theorem), we have

$$
\prod_{\substack{\ell \mid N_{\text {split }} \\ \ell \text { odd }}} \frac{1}{2}(\ell-1) \prod_{\substack{\ell \mid N_{\text {nonsplit }} \\ \ell \text { odd }}} \frac{1}{2}(\ell-1) \prod_{\substack{\ell \mid N_{\text {add }} \\ \ell \text { odd } \\ \ell \equiv 1(\bmod 3)}} \frac{1}{2}(\ell+2)(\ell-1) \prod_{\substack{\ell \mid N_{\text {add }} \\ \ell \text { odd } \\ \ell \equiv 2(\bmod 3)}}(\ell-1) \prod_{4 \mid N_{\text {add }}} 2
$$


valid choices of residue classes for $m \bmod M$. Combining all the above and summing over each valid residue class $m \bmod M$, we immediately obtain our lower bound for the proportion of valid $L$ (with $M=9 M^{\prime}$ ).

For the second case (concerning imaginary quadratic fields), the asserted statement follows from taking $M$ as above, then choosing a positive integer $m$ such that

(1) $m \equiv-1(\bmod 3)$,

(2) $\ell$ odd prime, $\ell \mid N_{\text {split }} \Longrightarrow m \equiv$ [quadratic nonresidue unit] $(\bmod \ell)$,

(3) $2 \mid N_{\text {split }} \Rightarrow m \equiv 5(\bmod 8)$,

(4) $\ell$ odd prime, $\ell \mid N_{\text {nonsplit }} \Rightarrow m \equiv$ [quadratic residue unit] $(\bmod \ell)$,

(5) $2 \mid N_{\text {nonsplit }} \Longrightarrow m \equiv 1(\bmod 8)$,

(6) $\ell$ odd prime $, \ell \mid N_{\text {add }}, \ell \equiv 1(\bmod 3) \Longrightarrow m \equiv$ [quadratic nonresidue unit] $(\bmod \ell)$ or $m \equiv 0(\bmod \ell)$ and $m \neq \equiv\left(\bmod \ell^{2}\right)$,

(7) $\ell$ odd prime, $\ell \mid N_{\text {add }}, \ell \equiv 2(\bmod 3) \Longrightarrow m \equiv 0(\bmod \ell)$ and $m \neq \equiv 0\left(\bmod \ell^{2}\right)$,

(8) $4 \mid N_{\text {add }} \Longrightarrow m \equiv 8$ or $12(\bmod 16)$,

and proceeding by the same argument as above.

Theorem 54. Suppose $E / \mathbb{Q}$ is any elliptic curve whose mod-3 Galois representation E[3] is reducible of type $\left(1,1, N_{\text {split }}, N_{\text {nonsplit }}, N_{\text {add }}\right)$, where 3 is a good prime of $E$. Let $L$ be any quadratic field such that

(1) 3 is inert in $L$,

(2) $3 \nmid h_{L \cdot \mathbb{Q}(\sqrt{-3})}$ if $L$ is real, and $3 \nmid h_{L}$ if $L$ is imaginary,

(3) $\ell \mid N_{\text {split }}$ implies $\ell$ is inert in $L$,

(4) $\ell \mid N_{\text {nonsplit }}$ implies $\ell$ is split in $L$,

(5) $\ell \mid N_{\text {add }}$ implies $\ell$ is inert in $L$ and $\ell \not \equiv 2(\bmod 3)$, or $\ell$ is ramified in $L$,

(6) $4 \mid N$ implies $D_{L} \equiv 8$ or $12(\bmod 16)$.

Let $M^{\prime}=\operatorname{lcm}\left(N, D_{L}^{2}\right)$ if $\operatorname{lcm}\left(N, D_{L}^{2}\right)$ is odd, $M^{\prime}=\operatorname{lcm}\left(N, D_{L}^{2}\right.$, 8) if $2 \| \operatorname{lcm}\left(N, D_{L}^{2}\right)$, and $M^{\prime}=\operatorname{lcm}\left(N, D_{L}^{2}, 16\right)$ if $4 \mid \operatorname{lcm}\left(N, D_{L}^{2}\right)$. Then if $L$ is real for a positive proportion of at least

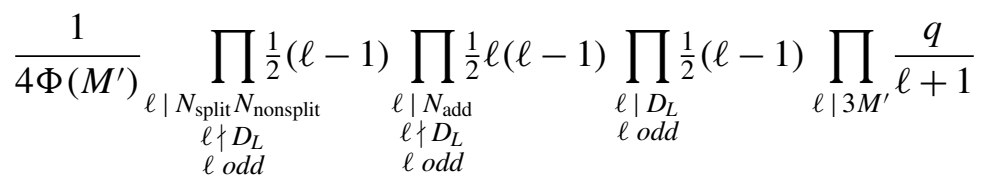


imaginary quadratic fields $K$, and if $L$ is imaginary for a positive proportion of at least

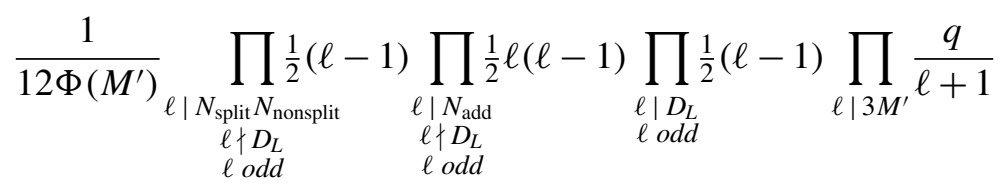

imaginary quadratic fields $K$, then $K$ satisfies the Heegner hypothesis with respect to $E_{L}$, we have $\left(D_{K}, D_{L}\right)=1$, and the Heegner point $P_{E_{L}}(K)$ is nontorsion. (Here, again, $q=4$ for $\ell=2$, and $q=\ell$ for odd primes $\ell$.)

Proof. Again we seek to apply Proposition 52, as well as Theorem 13. First suppose that $L$ is a real quadratic field. Let $M=3 \operatorname{lcm}\left(N, D_{L}^{2}\right)$ if $\operatorname{lcm}\left(N, D_{L}^{2}\right)$ is odd, $M=3 \operatorname{lcm}\left(N, D_{L}^{2}, 8\right)$ if $2 \| \operatorname{lcm}\left(N, D_{L}^{2}\right)$, and $M=3 \operatorname{lcm}\left(N, D_{L}^{2}, 16\right)$ otherwise. Using the Chinese remainder theorem, choose a positive integer $m$ such that

(1) $m \equiv 2(\bmod 3)$,

(2) $\ell$ odd prime, $\ell \mid N_{\text {split }} \Longrightarrow m \equiv$ [quadratic nonresidue unit] $(\bmod \ell)$,

(3) $2 \mid N_{\text {split }} \Longrightarrow m \equiv 5(\bmod 8)$,

(4) $\ell$ prime,$\ell \mid N_{\text {nonsplit }} \Rightarrow m \equiv$ [quadratic residue unit] $(\bmod \ell)$,

(5) $2 \mid N_{\text {nonsplit }} \Rightarrow m \equiv 1(\bmod 8)$,

(6) $\ell$ odd prime, $\ell \mid N_{\text {add }}, \ell \nmid D_{L} \Rightarrow m \equiv$ [quadratic nonresidue unit] $(\bmod \ell)$,

(7) $\ell$ odd prime, $\ell\left|N_{\text {add }}, \ell\right| D_{L} \Rightarrow m \equiv 0(\bmod \ell)$, where $m / D_{L} \equiv$ [quadratic residue unit] $(\bmod \ell)$,

(8) $4 \mid N \Longrightarrow m \equiv D_{L}(\bmod 16)$.

Suppose $K$ is any imaginary quadratic field with odd fundamental discriminant $D_{K}$ such that $\left(D_{L}, D_{K}\right)=1$ and $D_{L} D_{K} \equiv m(\bmod M)$. Since $D_{K}$ is odd, we must have $D_{K} \equiv 1(\bmod 4)$, and this is compatible with condition $(6)$ which forces $D_{K} \equiv 1(\bmod 8)$, which in turn forces 2 to split in $K$. Then the above congruence conditions on $m$, along with the congruence conditions of our assumptions, imply

(1) 3 is inert in $L$, split in $K$, and inert in $L \cdot K$,

(2) $\ell$ prime, $\ell \mid N_{\text {split }}, \ell \nmid D_{L} \Longrightarrow \ell$ is inert in $L$, split in $K$, and inert in $L \cdot K$,

(3) $\ell$ prime, $\ell \mid N_{\text {nonsplit }}, \ell \nmid D_{L} \Rightarrow \ell$ is split in $L$, split in $K$, and split in $L \cdot K$,

(4) $\ell$ odd prime, $\ell \mid N_{\text {add }}, \ell \nmid D_{L} \Rightarrow \ell$ is inert in $L$, split in $K$, and inert in $L \cdot K$,

(5) $\ell$ odd prime, $\ell \mid D_{L} \Longrightarrow \ell$ is ramified in $L$, split in $K$, and ramified in $L \cdot K$,

(6) $4 \mid N_{\text {add }} \Longrightarrow 2$ is ramified in $L$, split in $K$, and ramified in $L \cdot K$,

(7) if $2 \| N$, then $4 \mid M$ and $m \equiv 1(\bmod 4)$,

(8) if $4 \mid N$, then $16 \mid M$ and $m \equiv 8$ or $12(\bmod 16)$. 
Thus for imaginary quadratic $K$ such that $D_{L \cdot K}=D_{L} D_{K} \equiv m(\bmod M),(E, 3, L, K)$ satisfies all the congruence conditions of Theorem 13 except for possibly $3 \nmid h_{L \cdot K}$. Moreover, the congruence conditions above imply that $m$ and $M$ are valid positive integers for Theorem 51. Thus, by Proposition 52,

$$
\lim _{x \rightarrow \infty} \frac{\left|K_{*}^{-}(x, m, M)\right|}{\left|K^{-}(x, 1,1)\right|} \geq \frac{1}{2 \Phi(M)} \prod_{\ell \mid M} \frac{q}{\ell+1},
$$

so a positive proportion of imaginary quadratic $K$ satisfy $D_{L \cdot K} \equiv m(\bmod M)$ and $3 \nmid h_{L \cdot K}$. Thus, for these $K,(E, 3, L, K)$ satisfies all the congruence conditions of Theorem 13, and so $P_{E_{L}}(K)$ is nontorsion. Moreover, noticing that the congruence conditions (1)-(6) on $m$ above are independent (again by the Chinese remainder theorem), we have

$$
\prod_{\substack{\ell \mid N_{\text {split }} N_{\text {nonsplit }} \nmid D_{L} \\ \ell \text { odd }}} \frac{1}{\substack{\ell \mid N_{\text {add }} \\ \ell \nmid D_{L} \\ \ell \text { odd }}} \prod_{\substack{\ell \mid D_{L} \\ \ell \text { odd }}} \frac{1}{2} \ell(\ell-1)
$$

choices for residue classes of $m \bmod M$. Combining all the above and summing over each valid residue class $m \bmod M$, we immediately obtain our lower bound for the proportion of valid $K$ (with $M=3 M^{\prime}$ ).

For the case when $L$ is an imaginary quadratic field, let $M$ be as above. Then choose a positive integer $m$ such that

(1) $m \equiv 3(\bmod 9)$,

(2) $\ell$ odd prime, $\ell \mid N_{\text {split }} \Longrightarrow m \equiv-3$ [quadratic nonresidue unit] $(\bmod \ell)$,

(3) $2 \mid N_{\text {split }} \Rightarrow m \equiv 1(\bmod 8)$,

(4) $\ell$ odd prime, $\ell \mid N_{\text {nonsplit }} \Rightarrow m \equiv-3$ [quadratic residue unit] $(\bmod \ell)$,

(5) $2 \mid N_{\text {nonsplit }} \Rightarrow m \equiv 5(\bmod 8)$,

(6) $\ell$ odd prime, $\ell \mid N_{\text {add }}, \ell \nmid D_{L} \Rightarrow m \equiv-3$ [quadratic nonresidue unit] ( $\bmod \ell$ ),

(7) $\ell$ odd prime, $\ell \mid D_{L} \Longrightarrow m \equiv 0(\bmod \ell)$, where $m / D_{L} \equiv-3$ [quadratic residue unit] $(\bmod \ell)$,

(8) $4 \mid N \Longrightarrow m \equiv D_{L}(\bmod 16)$.

The argument then proceeds in the same way as above to establish $3 \nmid h_{L \cdot K \cdot \mathbb{Q}(\sqrt{-3})}$ and thus $\operatorname{rank}_{\mathbb{Z}} E_{L}(K)=1$ by applying Theorem 13 .

Proof of Corollary 18. Since E[3] is a reducible mod-3 Galois representation, $E$ has Eisenstein descent of type $\left(\psi, \psi^{-1}, N_{\text {split }}, N_{\text {nonsplit }}, N_{\text {add }}\right) \bmod 3$, where $\psi$ is some quadratic Dirichlet character. We may assume without loss of generality that $\psi=1$ (after possibly replacing $E$ by $E \otimes \psi^{-1}$ ). From Theorem 53, a positive proportion of quadratic twists $E_{L}$ satisfy the conditions of Theorem 54, and so 
by that theorem a positive proportion of imaginary quadratic $K$ have that $P_{E_{L}}(K)$ is nontorsion. If $E$ is semistable, then $E$ necessarily has Eisenstein descent of type $\left(1,1, N_{\text {split }}, N_{\text {nonsplit }}, N_{\text {add }}\right)$ by part (3) of Theorem 34 and by Theorem 35 . Since $N_{\text {add }}=1$, Theorems 53 and 54 produce twists $E_{L}$ with $\left(N, D_{L}\right)=1$ and $\operatorname{rank}_{\mathbb{Z}} E(K)=1$. Then by part (2) of Proposition 50, each $E_{L}$ has $w\left(E_{L}\right)=-\varepsilon_{L}(-1)$ and so $\operatorname{rank}_{\mathbb{Z}} E_{L}(\mathbb{Q})=\frac{1}{2}\left(1+\varepsilon_{L}(-1)\right)$ and $\operatorname{rank}_{\mathbb{Z}} E_{L \cdot K}(\mathbb{Q})=\frac{1}{2}\left(1-\varepsilon_{L}(-1)\right)$. The more precise lower bounds on these positive proportions follow immediately from Theorems 53 and 54.

Remark 55. There is no "double counting" resulting from using the lower bounds of Theorems 53 and 54 in tandem. The real quadratic twists $E_{L}$ produced in Theorem 53, which have discriminant $D_{L}$ prime to $D_{K}$, are distinct from the real twists $E_{L^{\prime} \cdot K}$ produced in Theorem 54 (with $L^{\prime}$ imaginary), which have discriminant $D_{L^{\prime}} D_{K}$. Similarly, the imaginary quadratic twists produced in Theorem 53 are distinct from those produced in Theorem 54.

Example 56. Let $E / \mathbb{Q}$ be the elliptic curve 19a1 in Cremona's labeling, which has minimal Weierstrass model

$$
y^{2}+y=x^{3}+x^{2}-9 x-15 .
$$

Then $E(\mathbb{Q})^{\text {tor }}=\mathbb{Z} / 3$, and so taking $p=3$, one sees that $E[3]$ is a reducible mod-3 Galois representation. Furthermore, $E$ has conductor $N=19$, where 19 is of split multiplicative reduction. Taking the real quadratic field $L=\mathbb{Q}(\sqrt{41})$, one can check that 3 and 19 are inert in $L$. Taking the imaginary quadratic field $K=\mathbb{Q}(\sqrt{-2})$, one sees that 3 splits in $K$ and that $K$ satisfies the Heegner hypothesis with respect to the quadratic twist $E_{L}$ (and 3 and 19 split in $K$ ). Furthermore, 3 does not divide the class numbers $h_{L \cdot \mathbb{Q}(\sqrt{-3})}=h_{\mathbb{Q}(\sqrt{-123})}=4$ and $h_{L \cdot K}=h_{\mathbb{Q}(\sqrt{-82})}=2$. Our result now gives $\operatorname{rank}_{\mathbb{Z}} E_{L}(K)=1$. By Proposition 50 , one sees that $\operatorname{rank}_{\mathbb{Z}} E_{L}(\mathbb{Q})=1$ and $\operatorname{rank}_{\mathbb{Z}} E_{L \cdot K}(\mathbb{Q})=0$. Taking the imaginary quadratic field $L^{\prime}=\mathbb{Q}(\sqrt{-7})$, one can check that 3 and 19 are inert in $L^{\prime}$. Furthermore, 3 does not divide the class numbers $h_{L^{\prime}}=1$ and $h_{L^{\prime} \cdot K \cdot \mathbb{Q}(\sqrt{-3})}=h_{\mathbb{Q}(\sqrt{-42})}=4$, so by Proposition 50 one sees that $\operatorname{rank}_{\mathbb{Z}} E_{L^{\prime}}(\mathbb{Q})=0$ and $\operatorname{rank}_{\mathbb{Z}} E_{L^{\prime} \cdot K}(\mathbb{Q})=1$. By Corollary 18 (and adding the explicit lower bounds given in Theorems 53 and 54 applied to $E, E_{L}$ and $E_{L^{\prime}}$ ), at least $\frac{19}{640}+\frac{19}{10240}=\frac{323}{10240}$ real quadratic twists of $E$ have rank 1 and at least $\frac{57}{640}+\frac{19}{17920}=\frac{323}{3584}$ imaginary quadratic twists of $E$ have rank 0 .

\section{Acknowledgements}

The author thanks Chris Skinner for suggesting this investigation and for helpful discussions and is indebted to Shou-Wu Zhang and Barry Mazur for valuable discussions. The author also thanks the anonymous referees for their insightful comments and suggestions. This work was partially supported by the National 
Science Foundation under grant DGE 1148900. Parts of the research contributing to this paper were completed while the author was a participant in the 2013 Princeton Summer Research Program.

\section{References}

[Atkin and Lehner 1970] A. O. L. Atkin and J. Lehner, "Hecke operators on $\Gamma_{0}(m)$ ", Math. Ann. 185 (1970), 134-160. MR 42 \#3022 Zbl 0177.34901

[Balsam 2014] N. Balsam, "The parity of analytic ranks among quadratic twists of elliptic curves over number fields", preprint, 2014. arXiv http://arxiv.org/abs/1404.4964

[Bertolini and Darmon 2005] M. Bertolini and H. Darmon, "Iwasawa's main conjecture for elliptic curves over anticyclotomic $\mathbb{Z}_{p}$-extensions", Ann. of Math. (2) 162:1 (2005), 1-64. MR 2006g:11218 Zbl 1093.11037

[Bertolini et al. 2013] M. Bertolini, H. Darmon, and K. Prasanna, "Generalized Heegner cycles and p-adic Rankin L-series”, Duke Math. J. 162:6 (2013), 1033-1148. MR 3053566 Zbl 1302.11043

[Bertolini et al. 2015] M. Bertolini, H. Darmon, and K. Prasanna, "p-adic L-functions and the coniveau filtration on Chow groups", J. Reine Angew. Math. (online publication April 2015), 1-66.

[Billerey and Menares 2013] N. Billerey and R. Menares, "On the modularity of reducible mod $l$ Galois representations”, preprint, 2013. arXiv 1309.3717

[Davenport and Heilbronn 1971] H. Davenport and H. Heilbronn, "On the density of discriminants of cubic fields, II”, Proc. Roy. Soc. London Ser. A 322:1551 (1971), 405-420. MR 58 \#10816 Zbl 0212.08101

[Deligne 1971] P. Deligne, "Formes modulaires et représentations $l$-adiques", exposé no. 355, 139-172 in Séminaire Bourbaki 1968/69, Lecture Notes in Math. 175, Springer, Berlin, 1971. MR 3077124 Zbl 0206.49901

[Deligne and Serre 1974] P. Deligne and J.-P. Serre, "Formes modulaires de poids 1", Ann. Sci. École Norm. Sup. (4) 7 (1974), 507-530. MR 0379379 Zbl 0321.10026

[Deninger 1990] C. Deninger, "Higher regulators and Hecke $L$-series of imaginary quadratic fields, II”, Ann. of Math. (2) 132:1 (1990), 131-158. MR 91i:19003 Zbl 0721.14005

[Diamond 1979] J. Diamond, "On the values of $p$-adic $L$-functions at positive integers", Acta Arith. 35:3 (1979), 223-237. MR 80j:12013 Zbl 0463.12007

[Diamond and Shurman 2005] F. Diamond and J. Shurman, A first course in modular forms, Graduate Texts in Mathematics 228, Springer, New York, 2005. MR 2006f:11045 Zbl 1062.11022

[Dokchitser 2013] T. Dokchitser, "Notes on the parity conjecture", pp. 201-249 in Elliptic curves, Hilbert modular forms and Galois deformations, edited by H. Darmon et al., Birkhäuser/Springer, Basel, 2013. MR 3184338 Zbl 1323.11047

[Geisser 1997] T. Geisser, " $p$-adic $K$-theory of Hecke characters of imaginary quadratic fields and an analogue of Beilinson's conjectures”, Duke Math. J. 86:2 (1997), 197-238. MR 97m:11086 Zbl 0899.11056

[Gelbart 1975] S. S. Gelbart, Automorphic forms on adèle groups, Annals of Mathematics Studies 83, Princeton University Press, Princeton, N.J., University of Tokyo Press, Tokyo, 1975. MR 52 \#280 Zbl 0329.10018

[Gouvêa 1988] F. Q. Gouvêa, Arithmetic of p-adic modular forms, Lecture Notes in Mathematics 1304, Springer, Berlin, 1988. MR 91e:11056 Zbl 0641.10024 
[Gross 1980] B. H. Gross, "On the factorization of p-adic L-series", Invent. Math. 57:1 (1980), 83-95. MR 82a:12010 Zbl 0472.12011

[Gross 1990] B. H. Gross, "A tameness criterion for Galois representations associated to modular forms $(\bmod p)$ ", Duke Math. J. 61:2 (1990), 445-517. MR 91i:11060 Zbl 0743.11030

[Harron and Snowden 2015] R. Harron and A. Snowden, "Counting elliptic curves with prescribed torsion”, J. Reine Angew. Math. (online publication January 2015), 1-20.

[Hida and Tilouine 1993] H. Hida and J. Tilouine, "Anti-cyclotomic Katz $p$-adic $L$-functions and congruence modules", Ann. Sci. École Norm. Sup. (4) 26:2 (1993), 189-259. MR 93m:11044 Zbl 0778.11061

[Katz 1976] N. M. Katz, " $p$-adic interpolation of real analytic Eisenstein series”, Ann. of Math. (2) 104:3 (1976), 459-571. MR 58 \#22071 Zbl 0354.14007

[Katz 1978] N. M. Katz, “p-adic L-functions for CM fields”, Invent. Math. 49:3 (1978), 199-297. MR 80h:10039 Zbl 0417.12003

[Kolyvagin 1990] V. A. Kolyvagin, "Euler systems", pp. 435-483 in The Grothendieck Festschrift, vol. II, edited by P. Cartier et al., Progr. Math. 87, Birkhäuser, Boston, 1990. MR 92g:11109 Zbl 0742.14017

[Li 2014] Z. K. Li, "Quadratic twists of elliptic curves with 3-Selmer rank 1", Int. J. Number Theory 10:5 (2014), 1191-1217. MR 3231410 Zbl 1297.14037

[Liu et al. 2014] Y. Liu, S. Zhang, and W. Zhang, "On p-adic Waldspurger Formula”, preprint, 2014, available at https://web.math.princeton.edu/ shouwu/publications/Coleman.pdf.

[Loeffler and Weinstein 2012] D. Loeffler and J. Weinstein, "On the computation of local components of a newform”, Math. Comp. 81:278 (2012), 1179-1200. MR 2012k:11064 Zbl 06028404

[Mazur 1978] B. Mazur, "Rational isogenies of prime degree", Invent. Math. 44:2 (1978), 129-162. MR 80h:14022 Zbl 0386.14009

[Mazur 1979] B. Mazur, "On the arithmetic of special values of $L$ functions", Invent. Math. 55:3 (1979), 207-240. MR 82e:14033 Zbl 0426.14009

[Nakagawa and Horie 1988] J. Nakagawa and K. Horie, "Elliptic curves with no rational points", Proc. Amer. Math. Soc. 104:1 (1988), 20-24. MR 89k:11113 Zbl 0663.14023

[Robert 1973] G. Robert, Unités elliptiques, Société Mathématique de France, Paris, 1973. MR 57 \#9669 Zbl 0314.12006

[Scholl 1990] A. J. Scholl, "Motives for modular forms", Invent. Math. 100:2 (1990), 419-430. MR 91e:11054 Zbl 0760.14002

[Serre 1972] J.-P. Serre, "Propriétés galoisiennes des points d'ordre fini des courbes elliptiques", Invent. Math. 15:4 (1972), 259-331. MR 52 \#8126 Zbl 0235.14012

[Serre 1973] J.-P. Serre, "Congruences et formes modulaires (d'après H. P. F. Swinnerton-Dyer)", exposé no. 416, 319-338 in Séminaire Bourbaki, 1971/1972, Lecture Notes in Math. 317, Springer, Berlin, 1973. MR 57 \#5904a Zbl 0276.14013

[Silverman 2009] J. H. Silverman, The arithmetic of elliptic curves, 2nd ed., Graduate Texts in Mathematics 106, Springer, Dordrecht, 2009. MR 2010i:11005 Zbl 1194.11005

[Stark 1977] H. M. Stark, "Class fields and modular forms of weight one”, pp. 277-287. Lecture Notes in Math., Vol. 601 in Modular functions of one variable, V (Proc. Second Internat. Conf., Univ. Bonn, 1976), edited by J.-P. Serre and D. B. Zagier, Springer, Berlin, 1977. MR 56 \#8539 Zbl 0363.12010

[Taya 2000] H. Taya, "Iwasawa invariants and class numbers of quadratic fields for the prime 3", Proc. Amer. Math. Soc. 128:5 (2000), 1285-1292. MR 2000j:11162 Zbl 0958.11069 
[Vatsal 1999] V. Vatsal, “Canonical periods and congruence formulae”, Duke Math. J. 98:2 (1999), 397-419. MR 2000g:11032 Zbl 0979.11027

[Washington 1997] L. C. Washington, Introduction to cyclotomic fields, 2nd ed., Graduate Texts in Mathematics 83, Springer, New York, 1997. MR 97h:11130 Zbl 0966.11047

[Yoo 2015] H. Yoo, "Nonoptimal levels of a reducible mod $l$ modular representation", preprint, 2015. arXiv 1409.8342

Communicated by Kiran S. Kedlaya

Received 2014-12-10 Revised 2015-12-12 Accepted 2015-12-15

dkriz@princeton.edu Department of Mathematics, Princeton University, Fine Hall, Washington Rd, Princeton, NJ 08544, United States 


\section{Algebra \& Number Theory}

msp.org/ant

\section{EDITORS}

MANAGING EDITOR

Bjorn Poonen

Massachusetts Institute of Technology

Cambridge, USA

\author{
EDITORIAL BOARD CHAIR \\ David Eisenbud \\ University of California \\ Berkeley, USA
}

BOARD OF EDITORS

Georgia Benkart

Dave Benson

Richard E. Borcherds

John H. Coates

J-L. Colliot-Thélène

Brian D. Conrad

Hélène Esnault

Hubert Flenner

Sergey Fomin

Edward Frenkel

Andrew Granville

Joseph Gubeladze

Roger Heath-Brown

Craig Huneke

Kiran S. Kedlaya

János Kollár

Yuri Manin

Philippe Michel
University of Wisconsin, Madison, USA

University of Aberdeen, Scotland

University of California, Berkeley, USA

University of Cambridge, UK

CNRS, Université Paris-Sud, France

Stanford University, USA

Freie Universität Berlin, Germany

Ruhr-Universität, Germany

University of Michigan, USA

University of California, Berkeley, USA

Université de Montréal, Canada

San Francisco State University, USA

Oxford University, UK

University of Virginia, USA

Univ. of California, San Diego, USA

Princeton University, USA

Northwestern University, USA

École Polytechnique Fédérale de Lausanne
Susan Montgomery

Shigefumi Mori

Raman Parimala

Jonathan Pila

Anand Pillay

Victor Reiner

Peter Sarnak

Joseph H. Silverman

Michael Singer

Vasudevan Srinivas

J. Toby Stafford

Ravi Vakil

Michel van den Bergh

Marie-France Vignéras

Kei-Ichi Watanabe

Efim Zelmanov

Shou-Wu Zhang
University of Southern California, USA

RIMS, Kyoto University, Japan

Emory University, USA

University of Oxford, UK

University of Notre Dame, USA

University of Minnesota, USA

Princeton University, USA

Brown University, USA

North Carolina State University, USA

Tata Inst. of Fund. Research, India

University of Michigan, USA

Stanford University, USA

Hasselt University, Belgium

Université Paris VII, France

Nihon University, Japan

University of California, San Diego, USA

Princeton University, USA

\section{PRODUCTION}

production@msp.org

Silvio Levy, Scientific Editor

See inside back cover or msp.org/ant for submission instructions.

The subscription price for 2016 is US $\$ 290 /$ year for the electronic version, and $\$ 485 /$ year ( $+\$ 55$, if shipping outside the US) for print and electronic. Subscriptions, requests for back issues and changes of subscribers address should be sent to MSP.

Algebra \& Number Theory (ISSN 1944-7833 electronic, 1937-0652 printed) at Mathematical Sciences Publishers, 798 Evans Hall \#3840, c/o University of California, Berkeley, CA 94720-3840 is published continuously online. Periodical rate postage paid at Berkeley, CA 94704, and additional mailing offices.

ANT peer review and production are managed by EditFLOW ${ }^{\circledR}$ from MSP.

\section{PUBLISHED BY}

- mathematical sciences publishers

nonprofit scientific publishing

http://msp.org/

(C) 2016 Mathematical Sciences Publishers 


\section{Algebra \& Number Theory}

Volume $10 \quad$ No. $2 \quad 2016$

Kummer theory for Drinfeld modules

RICHARD PINK

Parity and symmetry in intersection and ordinary cohomology

SHENGHAO SUN and WeIZHE ZHENG

DANIEL KRIZ

Squarefree polynomials and Möbius values in short intervals and arithmetic progressions JONATHAN P. KEATING and ZEEV RUDNICK

Equidistribution of values of linear forms on a cubic hypersurface SAM CHOW 